Maria Regina Alves Cardoso

\title{
COMPARAÇÃO ENTRE TRÊS MÉTODOS DE AMOSTRAGEM QUE VISAM À ESTIMAÇÃO DA COBERTURA VACINAL
}

Dissertação de mestrado apresentada à Faculdade de Saúde Pública de São Paulo-Departamento de Epidemiologia Prof. Orientador: Jair Lício Ferreira Santos

São Paulo 1990

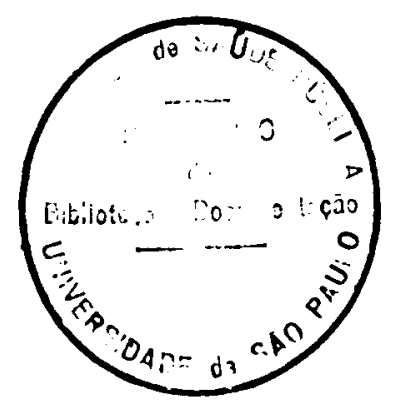


A Lídia e Antônio, meus pais. 
Agradecimentos

Ana Sílvia, Antônio, Antônio Carlos, Carlos, Carlos Alberto, Carlos Monteiro, Cássia, Cecília, Chester, Cícero, Cinit, Clâudia, Clemência, Clô, Dalva, Davi, Déia, Eliseu, Ernesto, Eunice, Eurivaldo, Fernanda, Fernão, Flávia, Frei Guilherme, Frei Jorge, Gabriel, Gerusa, Guilherme, Jair, Joselita, Josē Luiz, José Maria, Juarez, Lelé, Leonor, Lídia, Lúcia, Luís, Maggy, Mārcia, Marcos, Maria, Maria do Carmo, Maria Zilda, Nair, Neusa, Paulina, Rita, Roberto, Rosário, Sabina, Sérgio, Silvia, Silvinha, Tereza, Thaís, Vera, Vera Gattās, Victor. 


\section{RESUMO}

É apresentado um estudo comparativo entre três métodos de amostragem que visam à estimação da cobertura vacinal. 0 primeiro método, de R.H. Henderson e T. Sundaresan, utilizado pelo Programa Ampliado de Imunizações (PAI) da Organização Mundial de Saúde, é comparado com dois outros métodos alternativos brasileiros: o método de C.L. Szwarcwald e J.G. Valente, da Escola Nacional de Saúde Pública, Fundação Oswaldo Cruz, do Rio de Janeiro e o método eqüiprobabilístico de E.P. de C. Silva, da Faculdade de Saúde Pública da Universidade de São Paulo, São Paulo.

Foram constituidas quatro populações hipotéticas através de simulação em computador. Todas as possíveis amostras de cada população foram selecionadas utilizando-se os três procedimentos de amostragem com o objetivo de compará-los quanto à precisão, ao vício e ao erro quadrático médio. Os três métodos não apresentaram diferenças significativas e, embora o método de Henderson e Sundaresan não seja eqüiprobabilístico em condiçôes normais, é o mais simples e o menos dispendioso e, portanto, é recomendado para levantamentos de cobertura vacinal. 


\section{SUMMARY}

A comparative study among three sampling methods for estimating vaccinal coverage is presented. The first one, of R.H. Henderson and T. Sundaresan, used by the Expanded Programme on Immunization (EPI) of the World Health Organization, was compared with two alternative Brazilian sampling methods: one C.L. Szwarcwald and J.G. Valente, from the National School of Public Health, Oswaldo Cruz Foundation, from Rio de Janeiro; and another eqüiprobabilistic of E.P. de C. Silva, from de School of Public Health, University of São Paulo, São Paulo.

Four hypothetical populations were designed through computer simulation. All possible samples of each population were selected using three sampling procedures with the purpose of comparing them for precision, bias and mean square error. The three methods did not show significant differences. Although the Henderson and Sundarensan's method is not eqüiprobabilistic in normal conditions, it is the simplest and the least expensive and, therefore is recommended for vaccinal coverage surveys. 


\section{ÍNDICE}

1. Introdução $\ldots \ldots \ldots \ldots \ldots \ldots \ldots \ldots \ldots \ldots \ldots$

2. Objetivos $\ldots \ldots \ldots \ldots \ldots \ldots \ldots \ldots \ldots \ldots \ldots, 4$

3. Os Métodos de Amostragem para Estimação da Cobertura Vacinal .................. 5

3.1 Base Teórica .................... 5

3.2 Os Três Métodos de Amostragem ....... 12

3.2.1 Método de Henderson e Sundaresan . 12

3.2.2 Método de Szwarcwald e Valente ... 16

3.2.3 Método de Silva ............. 17

4. Material e Método .................... 22

4.1 Desenho da Simulação .............. 22

4.2 Processo da Simulação ............. 27

4.2.1 Os conglomerados e as subamostras . 27

4.2.2 As amostras de 30 conglomerados .. 30

4.3 Análise dos Dados ................ 30

5. Resultados e Discussão .................. 33

6. Conclusões ........................... 35 
7. Referências Bibliográficas .............. 36

8. Anexos .......................... 38 


\section{INTRODUÇÃO}

Um dos grandes desafios para a maioria dos países tem sido a questão da melhoria dos niveis de saúde de suas populaçóes. 0 ideal almejado pelos países em desenvolvimento é a constituição ou o aperfeiçoamento de sistemas nacionais de saúde completos, articulados com outros setores, de tal forma que as atividades do setor saúde sejam parte integrante do processo de desenvolvimento.

0 gerenciamento de sistemas desta natureza depende, em grande medida, da disponibilidade de informações para o planejamento, o monitoramento e para a avaliação da eficiência e do impacto dos programas desenvolvidos.

Cada nível de um sistema de saúde tem suas necessidades próprias em matéria de informação. Pode ocorrer que os dados devam ter diversos graus de elaboração ou que estejam agrupados de maneira distintas, mas de qualquer forma, a obtenção e a análise dos dados necessários são tarefas das equipes de profissionais do setor saúde.

Muitas vezes as informações requeridas podem ser obtidas utilizando-se dados normalmente registrados, mas em outras ocasiões é preciso realizar levantamentos de dados existentes porém não registrados ${ }^{8}$ para que se possa estudar com mais propriedade o fenômeno de interesse.

A Organização Mundial de Saúde (OMS) tem incentivado o uso de tais levantamentos. Isto pode ser observado, por exemplo, no Programa Ampliado de Imunizações (PAI).

Este programa, proposto em 1.974 , é o resultado de um trabalho conjunto empreendido pela OMS, pela Organização Panamericana de Saúde (OPS) e pelo Fundo das Naçôes Unidas para a Infância (UNICEF) e tem como objetivo reduzir a morbidade e a mortalidade através da imunização contra as seis doenças de maior incidência entre as crianças do mundo: sarampo, poliomielite, coqueluche, tétano, difteria e tuberculose.

Para que se possa ampliar a cobertura vacinal o PAI sistematiza, baseado em princípios técnicos e científicos, todas as atividades envolvidas nesta ação, desde o treinamento de pessoal até os procedimentos de avaliação do programa.

Em relação à avaliação da cobertura vacinal, o PAI desenvolveu uma metodologia de levantamento de dados em nível domiciliar que utiliza uma técnica de amostragem simples, podendo ser executada pelo pessoal de nível 
local, de baixo custo e que produz resultados rapidamente ${ }^{22}$. Este método foi aplicado inicialmente nos Estados Unidos e mais tarde em comunidades do oeste da África ${ }^{4} \mathrm{e}$ em outros países ${ }^{17}$.

No Brasil já foram realizados levantamentos com uso desta metodologia em Araraquara, Recife e São Paulo ${ }^{(13,15)}$.

Devido à simplicidade e ao custo reduzido este procedimento tem sido aplicado ultimamente com outros propósitos além do conhecimento da cobertura vacinal ${ }^{16}$. Entretanto, surgiu no decorrer do tempo uma certa polêmica entre os estatísticos a respeito da eficiência estatística deste método e com isto tem surgido trabalhos publicados no sentido de discutir e avaliar a técnica de amostragem proposta pelo $P A I^{(9,10,12)}$ e ainda, tem sido propostos métodos alternativos para levantamento de cobertura vacinal que têm como objetivo minimizar ou eliminar problemas potenciais deste método.

Esta dissertação pretende comparar a técnica de amostragem do PAI com duas outras alternativas propostas por C. L. Szwarcwald e J.C.Valente ${ }^{21}$, da Escola Nacional de Saúde Pública - FIOCRUZ e Departamento de Vigilância Epidemiológica da Secretaria de Estado de Saúde e Higiene do Rio de Janeiro respectivamente, e por E. P. de C. Silva ${ }^{20}$, da Faculdade de Saúde Pública da Universidade de São Paulo.

Considerando que as três técnicas de amostragem são probabilísticas, isto é, todos os elementos da população em estudo possuem probabilidade conhecida e diferente de zero de pertencer à amostra ${ }^{7}$, torna-se possível aplicar a teoria da amostragem para a comparação destes métodos. Assim, a proposta desta dissertação é trabalhar com a distribuição amostral do estimador da cobertura vacinal, ou seja, obtendo-se de uma população todas as possíveis amostras de mesmo tamanho e com o mesmo procedimento de amostragem pode-se definir a distribuição de freqüência das estimativas do valor populacional.

Conhecendo-se esta distribuição amostral para cada método de amostragem é possivel comparar as técnicas quanto à precisão, ao vício e a exatidão.

A precisão é dada pela variância da distribuição amostral, enquanto que, o vício indica a diferença entre o valor esperado de uma estatística e o valor populacional. 0 erro quadrático médio, por outro lado, mede a exatidão do procedimento de amostragem, ou seja, ele considera a precisão e o vício conjuntamente. A Figura 1.1 representa estes conceitos esquematicamente.

Para que seja factível esta forma de comparação, as populações e as amostras estudadas neste trabalho são fruto de simulações realizadas em compu- 
tador. 
Figura 1.1. Representação esquemótica do distribuição amostral do estimador $\widehat{\boldsymbol{\theta}}$

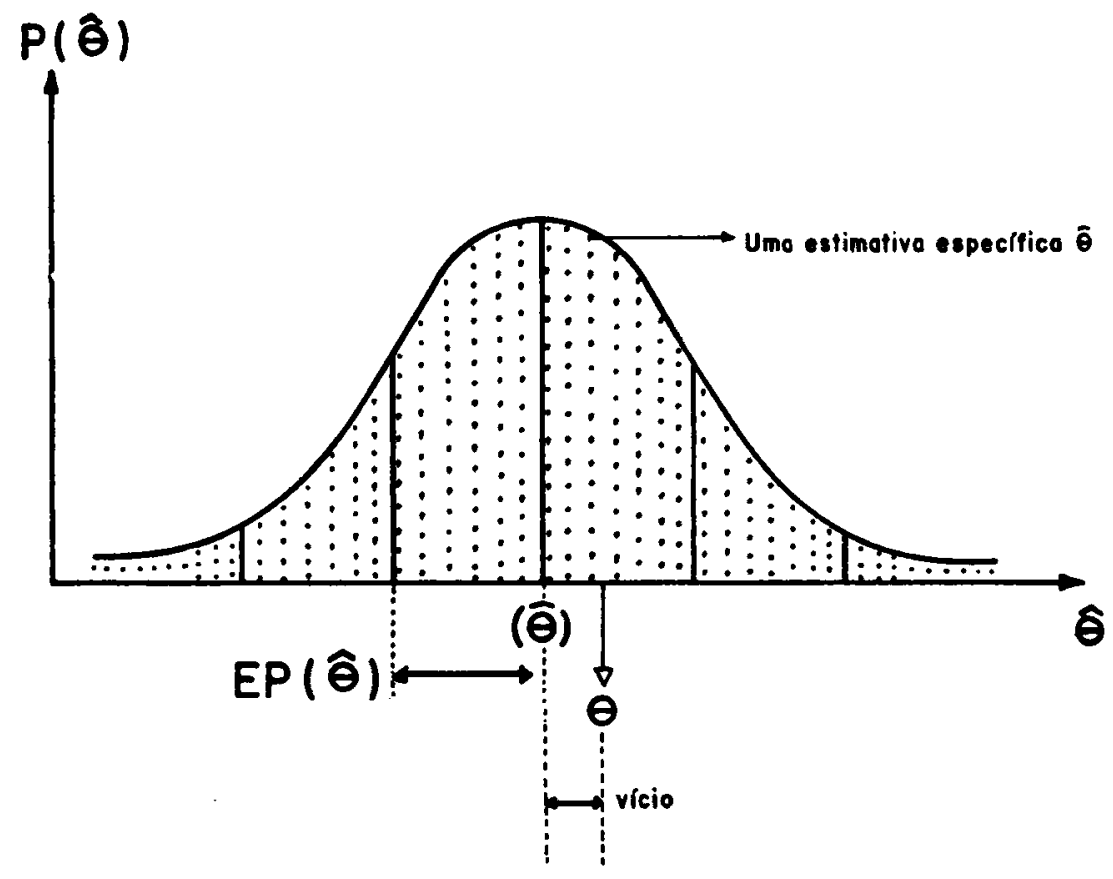

$\theta=$ valor populacional

$E(\bar{\theta})$ esperança do estimador $\bar{\theta}$

$\operatorname{EP}(\bar{\theta})=$ orro padrão do estimador $\bar{\theta}$

$\operatorname{EP}(\bar{\theta})=\sqrt{\operatorname{var}(\bar{\theta})}$

$P(\bar{\theta})=$ probabilidade de $\bar{\theta}$

vício $(\bar{\theta})=E(\bar{\theta})-\theta$

$\operatorname{EQM}(\bar{\theta})=\operatorname{var}(\bar{\theta})+(\operatorname{vicio}(\bar{\theta}))^{2}$

Obs: a distribuiçð̄o omostral of oproximadamente normal com tamanhos moderados de amostra paro a moioria das estatisticas. 


\section{OBJETIVOS}

Os objetivos deste trabalho são:

1. Comparar quanto à precisão na estimação da cobertura vacinal, para fixado tamanho de amostra, os seguintes procedimentos de amostragem:

- Método de Henderson e Sundaresan (PAI)

- Método de Szwarcwald e Valente

- Método de Silva

2. Comparar quanto ao vício os procedimentos de amostragem relacionados no objetivo número 1 .

3. Comparar quanto ao erro quadrático médio os procedimentos de amostragem relacionados no objetivo número 1 . 


\section{OS MÉTODOS DE AMOSTRAGEM PA- RA ESTIMAÇÃO DA COBERTURA VA- CINAL}

\subsection{Base Teórica}

Um método de amostragem deve contemplar dois aspectos: o processo de seleção, que define as regras e operações a serem realizadas para a seleção dos elementos que participarão de uma particular amostra e o processo de estimação que especifica a função matemática (estimador) utilizada para a obtenção das estimativas amostrais de valores populacionais ${ }^{7}$.

Os processos de seleção empregados pelos três métodos de amostragem analisados neste trabalho estão apoiados na teoria da Amostragem por Conglomerados. Este procedimento de amostragem probabilística é aplicado quando a seleção de elementos individuais é dificultada pela impossibilidade de se construir rapidamente listas de todos os elementos que constituem a população (sistema de referência) ou pela distância a ser percorrida entre os elementos selecionados, que podem estar muito espalhados ou, ainda, por outras situaçóes que como as anteriores tornariam o levantamento extremamente dispendioso e portanto inviabilizado.

Nestes casos, a população pode ser dividida em subconjuntos de elementos, chamados conglomerados, que servem como unidades amostrais. Desta forma pode-se obter uma amostra de elementos selecionando-se uma parcela destes conglomerados e fazendo-se com que todos os elementos que os constituem participem da amostra. Isto leva a uma redução no custo por elemento amostrado porque diminui os gastos com a listagem das unidades amostrais e/ou com a localização dos elementos.

Os conglomerados são definidos de acordo com as necessidades impostas pela distribuição física da população e pela natureza do sistema de referência, mas tendo sempre em vista um desenho amostral economico e prático. Frequentemente os conglomerados naturalmente encontrados na maioria das populações são de tamanhos (número de elementos) diferentes, como por exemplo, domicílios em um quarteirão, individuos em uma família, alunos em uma sala de aula, etc.

Nesta técnica de amostragem apenas uma parte dos conglomerados que 
compõem a população fará parte da amostra. Estes conglomerados sclecionados devem representar aqueles que não o foram. Esta tarefa é bem sucedida quando cada um deles for internamente o mais heterogêneo possível em relação às variáveis de interesse no levantamento. Contudo, exceto em circunstâncias especiais, este objetivo não é plenamente alcançado e, assim, frequentemente existe um certo grau de homogeneidade, passível de mensuração, que traz como consequência uma perda em precisão nas estimativas realizadas por este tipo de amostra quando comparadas com àquelas calculadas a partir de amostras casuais simples de mesmo tamanho.

A magnitude deste chamado efeito do desenho é dada pela razão entre as variâncias estimadas da própria amostra por conglomerados e de uma amostra casual simples de mesmo tamanho, e esta razão depende do modo como os conglomerados são formados. Na maioria das situações práticas são utilizados os conglomerados naturalmente encontrados na população e a distribuição da população dentro destes em geral não é casual, ou seja, tem sido observado, principalmente em pesquisas sociais, que os elementos de um grupo tendem a ser semelhantes uns aos outros, possivelmente por fatores seletivos no agrupamento, por exposição a influências similares ou por efeitos de interação mútua ou, ainda, por uma combinação dessas três fontes ${ }^{7}$.

Desta maneira, em outras palavras, a homogeneidade destes grupos é maior do que seria se os indivíduos fossem designados ao acaso para comporem estes conglomerados, ocasionando assim uma perda em precisão da amostra evidenciada pelo acréscimo na variância estimada e, portanto, gerando uma medida de efeito do desenho maior que 1.

Os dois aspectos conflitantes - custo e precisão - nesta técnica de amostragem podem ser equacionados realizando-se uma subamostragem. Neste procedimento em primeiro lugar selecionam-se grandes conglomerados, que tendem a possuir uma taxa de homogeneidade menor e, em seguida, selecionamse elementos dentro de cada um destes conglomerados.

Este método é conhecido como amostragem por conglomerados em dois estágios de seleção, podendo ser prontamente estendido para mais estágios (amostragem em múltiplos estágios), bastando para isto uma hierarquia de diferentes tipos de unidades amostrais, cada unidade de primeiro estágio sendo dividida em unidades de segundo estágio, estas em unidades de terceiro estágio e assim sucessivamente.

As unidade amostrais do primeiro estágio são chamadas Unidades Primá- 
rias de Amostragem (UPA's) e elas são particularmente importantes para o cálculo da variância. As unidades dos estágios seguintes são denominadas Unidades Secundárias (USA's), Terciárias, etc.

É necessario um sistema de referência para cada estágio de seleção, porém, a partir do segundo estágio esta listagem é construida apenas para as unidades amostrais já selecionadas no estágio imediatamente anterior, por exemplo, se uma amostra de domicílios de uma determinada área for selecionada utilizando-se a técnica da amostragem em dois estágios, pode-se definir o quarteirào como sendo a UPA e listar todos os quarteirões da área a fim de selecionar um certo número deles, em seguida, listam-se todos os domicílios (USA's) somente dos quarteirões já selecionados na primeira etapa.

Nota-se que este tipo de procedimento proporciona uma economia na listagem e localização dos domicílios, pois estes estão confinados em alguns quarteirōes. Este método de amostragem em dois ou mais estágios tem grande flexibilidade, são permitidas variações em cada etapa de seleção, todavia, é conveniente, para os propósitos deste trabalho, ressaltar algumas características básicas que estão presentes em uma grande gama de desenhos frequentemente utilizados. Segundo Kish ${ }^{7}$ estas características são:

1. Seleção aleatória de uma amostra de UPA's do total de UPA's da população. A fração de amostragem $f_{1}=\frac{a}{A}$, onde $a$ indica o número de UPA's na amostra e $A$ o número de UPA's na população representa a probabilidade de seleção neste primeiro estágio. A seleção aqui pode ser sem reposição ou com reposição. Neste último caso a mesma unidade primária pode ser selecionada duas vezes e se isto ocorrer devem ser sorteadas duas subamostras separadas, cada uma contendo elementos diferentes.

2. Seleção, sem reposição, de uma subamostra com a mesma probabilidade, representada pela fração de amostragem $f_{2}$, dentro de cada UPA selecionada no primeiro estágio.

3. A fração global de amostragem, que representa a probabilidade de seleção de qualquer elemento da população, é dada pela expressão $f=f_{1}$ * $f_{2}$. Com esta fração global de amostragem se obtem uma amostra eqüiprobabilística, ou seja, todos os elementos da população têm a mesma probabilidade fixada de pertencer à amostra. 
4. Quando se aplica uma fração de amostragem $f_{2}$ constante a conglomerados de tamanhos iguais obtem-se subamostras de tamanhos iguais, contudo, na maior parte das situações práticas os conglomerados são de tamanhos diferentes e assim quando as UPA's são selecionadas com igual probabilidade $f_{1}$ e são selecionadas subamostras de tamanho fixado pode resultar em probabilidades diferentes $f=f_{1} * \frac{b}{B_{i}}$ (b: número de elementos na subamostra e $B_{i}$ : número total de elementos na $i$ ésima UPA). Isto significa que os elementos podem ser selecionados com probabilidades inversamente proporcionais ao tamanho da unidade primária e, portanto, a amostragem não seria eqüiprobabilística, o que não é prático nem eficiente em muitas situações. Para resolver esta questão a indicação é para manter a fração $f_{2}$ fixa, permitindo que o tamanho das subamostras variem.

Se a população a ser amostrada é constituida de conglomerados com tamanhos muito diferentes pode suceder que ao se permitir que $f_{2}$ seja constante - tamanho total da amostra obtida fique sujeito a grandes flutuações.

Embora o tamanho final da amostra raramente necessite ser exatamente especificado, até porque em muitas circunstâncias isto seria muito difícil devido à falta de informações precisas ou de procedimentos poderosos para controle do tamanho inicialmente planejado, é intuitivo que por razões financeiras, eficiência no trabalho de campo e, como se verá adiante, eficiência estatística este número total de elementos na amostra deva ficar dentro de limites aceitáveis.

Há vários modos de diminuir as variaçôes no tamanho da amostra, a seleção com probabilidade proporcional ao tamanho (PPT) é um destes.

Neste procedimento as UPA's são selecionadas com probabilidade proporcional aos seus tamanhos, ou seja, se uma UPA é 3 vezes maior que outra UPA, espera-se que ela seja 3 vezes mais freqüente na amostra do que esta última. Para que não ocorra nenhum vício na amostra por conta de UPA's super-representadas ou sub-representadas o procedimento estabelece que a fração de subamostragem para a i-ésima UPA selecionada seja igual a $\frac{f P}{a P_{i}}$, onde $P$ é a medida de tamanho para a população, $P_{i}$ é a medida de tamanho da i-ésima UPA e $a$ é o número de UPA's na amostra. $O$ tamanho total esperado da amostra é igual a $f N$, onde $N$ é o número de elementos na população. Este procedimento pode ser estendido para mais de duas etapas de amostragem, sendo que apenas no último estágio as unidades são selecionadas com 
igual probabilidade.

A seleção com PPT pode ser obtida fazendo-se uma listagem onde cada UPA está associada com seu respectivo tamanho e estes tamanhos são acumulados, como no exemplo:

$\begin{array}{cccccc}\text { UPA } & 1 & 2 & 3 & 4 & 5 \\ A_{i} & 30 & 70 & 50 & 26 & 40 \\ \text { Acumulado } & 30 & 100 & 150 & 176 & 216\end{array}$

As UPA's se relacionam com a linha dos valores acumulados da seguinte forma: para a UPA 1 são alocados 30 números de 001 a 030, para a UPA 2, os números de 031 a 100, para a UPA 3 os números de 101 a 150 e assim sucessivamente. Um número aleatório é selecionado entre 001 e 216 e a UPA correspondente ao intervalo que inclui este número aleatório é a UPA selecionada com PPT.

Este sorteio também pode ser realizado com a técnica da amostragem sistemática. Neste caso calcula-se o intervalo de amostragem dividindo-se o total acumulado pelo número de seleções a serem efetuadas. Supondo-se que se deseja sortear 3 UPA's, o intervalo de amostragem será $\frac{216}{3}=72$. Um número aleatório é selecionado - início casual - entre 01 e 72 para identificar a primeira UPA sorteada, a este início causal é somado o intervalo de amostragem para identificar a segunda UPA sorteada e a este segundo número soma-se novamente o intervalo para estabelecer a terceira UPA selecionada. Para exemplificar, de maneira bastante simples, se o início casual for igual a 10 ocorrerá que:

$\begin{array}{cc}\text { número selecionado } & \text { UPA sorteada } \\ 10 & 1 \\ (10+72)=82 & 2 \\ (82+72)=154 & 4\end{array}$

$\mathrm{Na}$ realidade raramente está disponível o verdadeiro tamanho das unidades de amostragem, mas por outro lado, pode-se obter estimativas desses valores através de censos recentes ou outras fontes. Quando estas estimativas são boas pode-se usá-las para o procedimento de seleção com PPT. Kalton ${ }^{6}$, todavia, considera importante distinguir quando estas medidas de tamanho estimadas são utilizadas, reservando para esta situação a expressão "probabilidade proporcional ao tamanho estimado" (PPTE). 
A fração global de amostragem para uma amostra eqüiprobabilística em dois estágios com PPTE pode ser escrita como:

$$
f=\frac{n}{N}=\left(\frac{a M_{i}}{\sum M_{i}}\right)\left(\frac{b}{M_{i}}\right)
$$

onde a é o número de UPA's selecionadas, $M_{i}$ é o tamanho estimado da i-ésima UPA e $b$ é um número tal que $\frac{a . b}{\sum M_{i}}=f$

A fração $\frac{b}{M_{i}}$ é aplicada no segundo estágio de seleção para proporcionar a eqüiprobabilidade da amostra, entretanto, nota-se que ao se aplicar esta taxa aos $B_{i}$ elementos da UPA i selecionada o tamanho esperado da subamostra para esta UPA será igual a $b\left(\frac{B_{i}}{M_{i}}\right)$. Este tamanho esperado oscilará de UPA para UPA dependendo da razão $\left(\frac{B_{i}}{M_{i}}\right)$ e será igual ao tamanho desejado $b$ somente quando $B_{i}=M_{i}$. Com boas estimativas de tamanho das unidades amostrais esta variabilidade no tamanho das subamostras não chega a comprometer o caracter eqüiprobabilístico deste procedimento de amostragem ${ }^{6}$.

Uma decorrência, portanto, do uso de PPTE é que o tamanho total da amostra não é fixo, mas antes é uma variável aleatória que depende das UPA's que foram selecionadas. O processo de estimação definido para este método de amostragem leva em conta esta característica e para enfatizá-la representa o tamanho total da amostra por $x$ em vez de $n$ e a média amostral por $r=\frac{z}{x}$, onde $y$ é o total na amostra para a variável $y$. Esta média amostral é denominada estimador-razão porque ela é uma razão de variáveis aleatórias ${ }^{6}$.

O estimador-razão é um estimador viciado da média populacional, mas este vício é desprezivel quando a variabilidade de $x$ é suficientemente pequena.

A variância do estimador-razão é complicada pelo fato que seu denominador é uma variável aleatória, sendo assim, na prática utiliza-se uma aproximação que calcula esta variância como se o denominador fosse constante ${ }^{7}$.

Para se assegurar, por um lado, uma pequena magnitude do vício deste estimador- razão de tal forma que possa ser ignorado e, por outro lado, a possibilidade de usar adequadamente a aproximação da variância de $r$, devese manter o coeficiente de variação de $x$ (definido como o erro-padrão de $x$ dividido pelo seu valor esperado) menor que 0,20 , mas preferencialmente menor que $0,10^{7}$.

Como já foi mencionado anteriormente, freqüentemente em desenhos de amostragem mais complexos ocorre uma perda em precisão, pensando-se 
neste fato surge a questão: "Como calcular o tamanho da amostra para garantir uma fixada precisão?". A resposta para este problema é composta de duas etapas: em primeiro lugar calcula-se o tamanho da amostra como se fosse para um procedimento de amostragem casual simples (A.C.S.), ou seja, no caso de variáveis binomiais a fórmula utilizada $\mathrm{e}^{(1)}$ :

$$
n^{\prime}=\frac{P \cdot Q \cdot Z_{(2)}^{2}}{d^{2}}
$$

onde

$n^{\prime}$ : tamanho da amostra para A.C.S.

$P$ : é a percentagem populacional

$Q: 1-\mathrm{P}$

$Z$ : valor da distribuição normal reduzida

$d$ : erro máximo em valor absoluto

O resultado obtido com a substituição dos valores nesta fórmula é, em seguida, multiplicado pelo efeito do desenho do delineamento mais complexo. Assim, tem-se:

$$
n=n^{\prime} * e f d
$$

onde

$n$ : tamanho da amostra para procedimento complexo

efd : efeito do desenho

Outro fator que necessita ser incluido no cálculo do tamanho da amostra é a ausência de resposta. Se a taxa de resposta prevista para um levantamento for de a\% deve-se selecionar uma amostra de tamanho $\frac{n}{a \%}$ para que se possa obter ao final da coleta de dados uma amostra de tamanho aproximadamente iqual ao desejado $(n)$.

\footnotetext{
"'não se está considerando o "fator de correção para populações finitas"

(2) tamanho da amostra é dado pela razão entre a variabilidade da variável de interesse na população $\left(\sigma_{\theta}^{2}\right)$ e a precisão desejada que é expressa pela variância da distribuição amostral do estimador $\left(\sigma_{\hat{\theta}}^{2}\right)$. Portanto, $n^{\prime}=\frac{\sigma_{\theta}^{2}}{\sigma_{\dot{\theta}}^{2}}$ onde $\sigma_{\hat{\theta}}^{2}$ pode ser expresso como $\frac{d^{2}}{Z^{2}}$ e $\sigma_{\theta}^{2}$ para variáveis binomiais é igual a P.Q .
} 


\subsection{Os Três Métodos de Amostragem}

Tendo como referência a teoria exposta anteriormente pode-se agora descrever de maneira mais sucinta os três métodos de amostragem para estimaşào da cobertura vacinal que estâo sendo comparados neste trabalho.

\subsubsection{Método de Henderson e Sundaresan (PAI)}

O método utilizado pelo Programa Ampliado de Imunizações consiste em:

a. identificação da área geográfica de interesse - esta área pode compreender uma população entre 50.000 e 1.000 .000 de habitantes ${ }^{11}$.

b. definição do grupo etário a ser avaliado quanto à situação vacinal

c. divisão da área geográfica a ser estudada em subáreas (unidades primárias de amostragem) - devem ser obtidas boas estimativas do tamanho, em termos de número de habitantes de cada subárea

d. seleção casual de 30 UPA's com probabilidade proporcional ao tamanho estimado

e. seleção casual de um "ponto de partida" (domicílio inicial) em cada UPA - o procedimento usado para selecionar o primeiro domicílio varia de acordo com a localização da área ( zona urbana ou rural) e com a disponibilidade de informações tais como listas de domicílios, mapas, etc.

O PAI recomenda que para áreas rurais onde existe uma listagem de domicílios da UPA se realize o sorteio de um número aleatório entre $1 \mathrm{e}$ o número total de domicílios da lista, inclusive. $O$ número selecionado corresponderá ao "domicílio inicial".

Para as áreas rurais onde não se dispõem destas listas de domicílios deve-se construi-las quando possivel, caso contrário, quando existem mais de 100 domicílios na UPA, deve-se escolher um ponto geograficamente central e selecionar ao acaso a direção na qual estará localizado o primeiro domicílio (por exemplo, fazendo-se a correspondência: 1 = norte; $2=$ sul; $3=$ leste e $4=$ oeste e sorteando-se um número entre 1 e 4, inclusive). Tendo selecionado a direção conta-se o número de 
domicílios existentes ao longo daquela linha direcional partindo-se do ponto central. Em seguida, seleciona-se um segundo número aleatório entre 1 e o número total de domicílios contados e este será o primeiro domicílio a ser visitado.

Quando a área é urbana a orientação é para verificar se existem subdivisões (geográfica, política) da UPA que contenham populações aproximadamente iguais. Se tais subdivisões existirem, deverão ser numeradas e uma delas selecionada aleatoriamente e esta será aquela na qual o domicílio inicial estará localizado. Se houver lista de domicílios para a subdivisão selecionada procede-se como na área rural onde existe esta listagem para a identificação do domicílio inicial. No caso onde não há a relação dos domicílios já preparada procede-se como descrito anteriormente para a área rural onde não há lista. Se por outro lado, não existirem estas subdivisões deve-se construi- las utilizando-se mapas da área e/ou realizando-se um rápido percurso pela região e, após este trabalho, seguir a mesma orientação dada para o caso onde não existem listas de domicílios para a seleção do "ponto de partida".

f. seleçâo de 7 crianças do grupo etário de interesse em cada uma das 30 UPA's - a seleção em uma UPA começa no domicílio inicial e continua pelos domicílios imediatamente seguintes, segundo um percurso préfixado, até que seja obtido um total de 7 crianças. Todas as crianças da idade especificada residentes no último domicílio pertencente à amostra são selecionadas mesmo que isto resulte em conglomerados de tamanhos superiores àquele requerido de 7 crianças.

Seguindo-se estas etapas obtem-se uma amostra de 210 crianças. Este tamanho de amostra proposto pelo PAI considera os seguintes dados:

1) o estado vacinal é tratado como uma variável binomial, isto significa que em relação a cada vacina ou dose de vacina de interesse as crianças são classificadas em duas categorias: vacinadas ou não vacinadas;

2) os valores de $P$ (proporção de crianças na população que são vacinadas) e $Q$ (proporção de não vacinadas) são 0,50 para ambas. Estes valores substituídos na fórmula [3.2] maximizam o valor de $n$; 
3) os valores assumidos por $Z$ e $d$ são, respectivamente, 1,96 e 0,10 . Isto significa que em $95 \%$ das possíveis amostras o erro amostral não ultrapassará $10 \%$, ou seja, se o valor populacional for $30 \%$, a cada 100 estimativas 95 delas estarão entre os valores $20 \%$ e $40 \%$;

4) admitindo um efeito do desenho igual a 2 (calculado a partir de levantamentos de cobertura vacinal para variola conduzidas anteriormente na África e Estados Unidos ${ }^{18}$ e substituindo-se na fórmula [3.3] chega-se a $n=192$;

5) aceitando-se que a seleção casual de 30 conglomerados é suficiente o bastante para assegurar que as médias dos conglomerados tenderão para uma distribuição normal ${ }^{1}$ permitindo o uso da teoria estatística baseada nesta distribuição para a análise de dados e ainda desejando-se a seleção de igual número de crianças em cada conglomerado torna-se necessário um tamanho de amostra igual a 210 crianças.

A probabilidade de seleção das crianças no método de Henderson e Sundaresan pode ser escrita como:

$1^{a}$ etapa :

$$
\frac{30 P_{i}}{P_{0}}
$$

onde $P_{i}$ representa a população da subárea i em determinado período de tempo $T$ e $P_{0}=\sum_{i}^{M} P_{i}$ que é a população total da área em estudo no periodo $T$ e $M$ é o número de UPA's que constituem a área de interesse. A fração [3.4] indica o número esperado de vezes que a subárea $i$ é selecionada para a amostra de 30 subáreas.

$2^{a}$ etapa :

$$
\frac{1}{D_{i}^{\prime}}
$$

onde $D_{i}^{\prime}$ é o número de domicílios na UPA $i$ no período do levantamento. 
Esta segunda fração [3.5] representa a probabilidade que cada domicílio da UPA $i$ tem de ser o domicilio inicial, dado que a UPA $i$ tenha sido selecionada na primeira etapa.

Consequentemente, a probabilidade que cada domicílio da área em estudo tem de ser um domicílio inicial é dada pela expressão:

$$
\frac{30 P_{i}}{P_{0}} \cdot \frac{1}{D_{i}^{\prime}}
$$

A expressão [3.6] terá sempre o mesmo valor f se a razão $\frac{P_{i}}{D_{i}^{\prime}}$ tiver sempre o mesmo valor para todas as $M$ subáreas. Se tal fato ocorrer pode-se substituir $P_{i}$ por $c D_{i}^{\prime}$ onde $c$ é uma constante ${ }^{20}$.

Nesta situação a expressão [3.6] pode se escrita como:

$$
\frac{30 c D_{i}^{\prime}}{P_{0}} \cdot \frac{1}{D_{i}^{\prime}}=\frac{30 c}{P_{0}}=f
$$

Considerando que todas as crianças residentes nos domicílios selecionados pertencerão à amostra, tem-se que a probabilidade de qualquer criança da área em estudo tem de participar da amostra é igual à probabilidade que tem o domicilio onde ela reside de ser selecionado. Todavia, mesmo admitindo que o valor da expressão [3.6] seja constante para todas as MUPA's, isto é, que qualquer domicílio da área de interesse tenha a mesma probabilidade de ser um domicílio inicial, ainda assim a probabilidade que uma criança residente na área estudada tem de pertencer à amostra pode não ser constante pois dependerá do número de domicílios sem crianças intercalados entre os com crianças, que precedem aquele em que reside a criança e ainda do número de crianças em um mesmo domicílio nos domicílios com crianças que precedem o desta.

Observando-se a Figura [3.1] e a Tabela [3.1] torna-se mais fácil verificar os fatos descritos anteriormente. Por exemplo, a probabilidade da criança residente no domicílio número 3 pertencer à subamostra de 7 crianças da UPA $i$ é igual a $\frac{23}{30}$, já a probabilidade da criança residente no domićlio número 17 é igual a $\frac{18}{30}$. 
Figuro 3.1 Distribuição dos domicilios do UPA $\mathrm{i}$
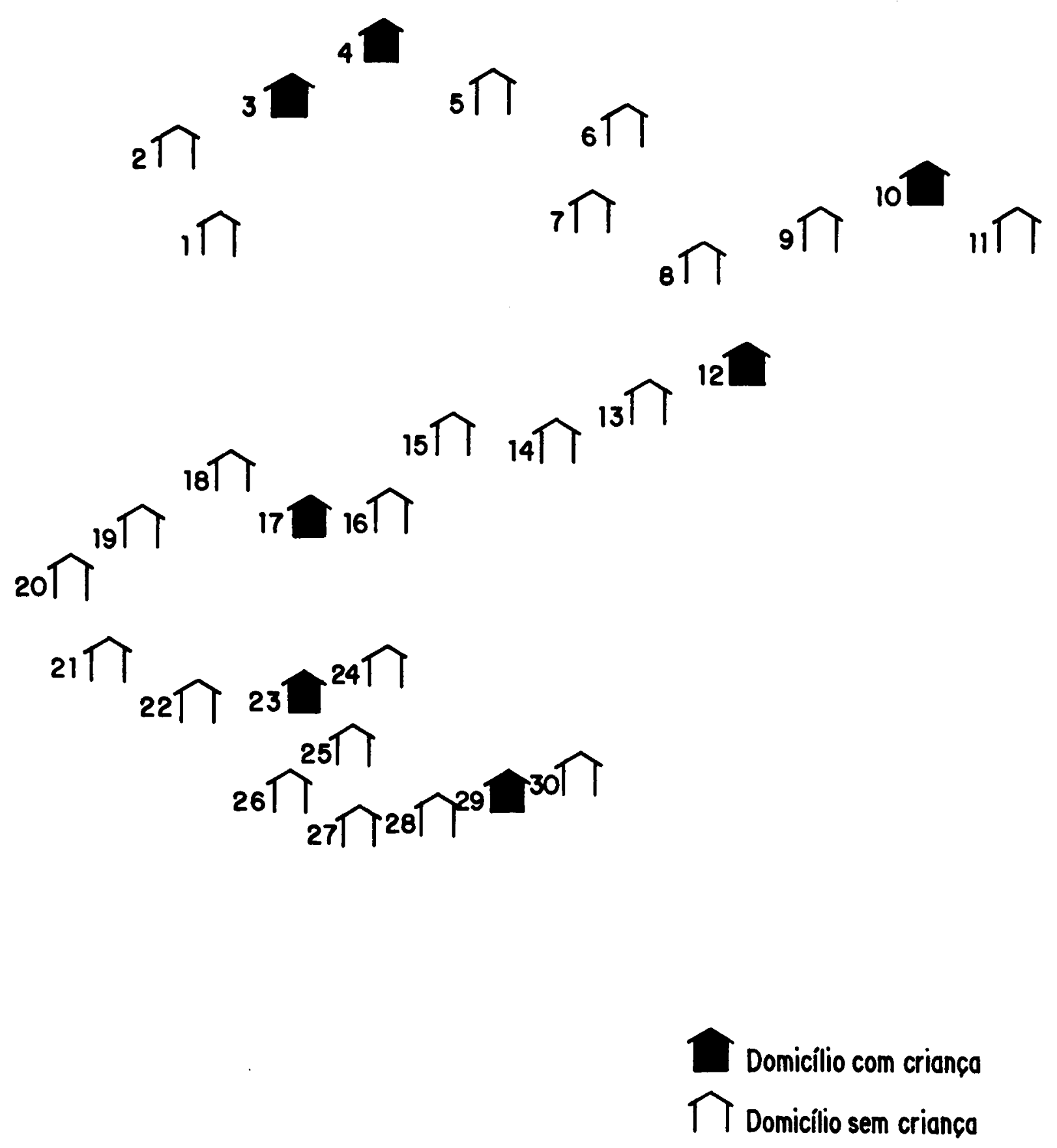
Tabela 3.1 - Relação dos domicílios com criança segundo o número de crianças, UPA $i$

\begin{tabular}{cc}
\hline domicílio & $n^{\circ}$ de crianças \\
\hline 3 & 1 \\
6 & 1 \\
10 & 2 \\
12 & 1 \\
17 & 1 \\
23 & 2 \\
29 & 2 \\
\hline Total & 10 \\
\hline
\end{tabular}

\subsubsection{Método de Szwarcwald e Valente}

A proposta deste método é realizar a primeira etapa de seleção da amostra da mesma maneira que o método de Henderson e Sundaresan, porém, na segunda etapa de seleção deve-se conduzir um pequeno censo em cada uma das 30 UPA's sorteadas com o intuito de identificar todos os domicílios com pelo menos uma criança da idade alvo residente. Após o arrolamento destes domicílios para cada UPA, 7 deles são sorteados de maneira sistemática.

A probabilidade com que cada criança residente na área de interesse é selecionada é dada pela expressão

$$
\frac{30 P_{i}}{P_{0}} \cdot \frac{7}{D_{c i}^{\prime}}
$$

onde $D_{c i}^{\prime}$ representa o número de domicílios com crianças na UPA $i$ no período do levantamento.

Haverá eqüiprobabilidade na seleção de crianças se as razôes $\frac{P_{i}}{D_{c i}^{\prime}}$ para as $M$ UPA's forem constantes. 


\subsubsection{Método de Silva}

Este método propõe que a amostragem seja eqüiprobabilística para que se possa utilizar adequadamente o estimador-razão, dado pela expressão [3.8], que é o indicado para se estimar a cobertura vacinal, assim como a fórmula para a sua variância

$$
r=\frac{\sum_{j=1}^{30} y_{j}}{\sum_{j=1}^{30} x_{j}}
$$

onde neste caso $\mathrm{r}$ representa a cobertura vacinal estimada, $x_{j}$ o número de crianças selecionadas na UPA $j$ e $y_{j}$ o número de crianças vacinadas dentre as $x_{j}$ sorteadas dessa UPA $j$.

Para este procedimento de amostragem devem ser efetuadas as seguintes operações:

1) Calcula-se a fração global de amostragem

$$
f=\frac{n}{\hat{C}}
$$

onde

$f:$ fração global de amostragem

$$
n=\frac{210}{a \%}
$$

$a \%$ : taxa de resposta em levantamento análogo

$\hat{C}$ : estimativa do total de crianças na faixa etária estudada no período em que se realiza o levantamento

Esta fração global de amostragem será aplicada aos domicílios com crianças na faixa de idade alvo residentes na área de interesse.

2) Divide-se a área em estudo em subáreas (UPA's). Esta divisão deve ser feita de tal maneira que a população total $P_{i}$ para cada UPA $i$ possua uma estimativa de crianças nela residentes que seja no mínimo o dobro do número de crianças que se pretende sortear em cada UPA, ou seja

$$
P_{i} * t \% \geq 14
$$

onde $t \%$ é a proporção que o grupo etário das crianças estudadas representa na população geral. 
3) Primeira etapa do levantamento: selecionam-se 30 UPA's com probabilidade proporcional ao tamanho estimado da UPA. A probabilidade de seleção de cada UPA é dada por

$$
\frac{30 D_{i}}{D_{0}}
$$

onde

$D_{i}$ : número de domicílios da UPA $i$ (tamanho estimado)

$D_{0}:=\sum_{i=1}^{M} D_{i}$ total de domicílios na área de interesse

$M$ : número total de UPA's

4) Segunda etapa do levantamento: considerando-se os domicílios de uma UPA $i$ selecionada na primeira etapa em determinada ordem, os domicílios com crianças conseqüentemente também ficarão ordenados. A estes domicílios com criança ordenados aplica-se amostragem sistemática com fração de amostragem

$$
\frac{b}{D_{i}}
$$

de tal forma que multiplicando-se [3.9] por [3.10] resulte a fração global de amostragem

$$
\frac{30 D_{i}}{D_{0}} \cdot \frac{b}{D_{i}}=f
$$

ou seja,

$$
b=\frac{f D_{0}}{30}
$$

observa-se que sempre se deve garantir que $D_{i} \geq b$. Para o sorteio sistemático de domicílios com crianças na UPA $i$ emprega-se o intervalo de amostragem $\frac{D_{i}}{b}$. Lembrando que estes domicílios estão ordenados, aqueles que pela respectiva numeração corresponderem aos números 
selecionados serão os domicílios com criança que constituirão a amostra. A autora deste método ressalta uma séric de observaçōes que servem como guia para a implementação da técnica proposta. Estas observações estão transcritas a seguir:

"1 - Se, na divisão de $D_{i}$ por b, não resultar número inteiro exato, faz-se a aproximação até a quarta casa decimal, se até alguma anterior a esta, não tiver sido exata a divisão.

$2^{a}-0$ começo casual deverá ter tantas casas decimais quantas forem as do intervalo.

$3^{a}$ - Os "números selecionados" são obtidos tomando-se o começo casual, com as suas casas decimais (se for o caso) e a ele somando-se sucessivamente $o$ intervalo (também com as suas casas decimais se for o caso); na seqüência de números que vão sendo obtidos, cada qual com as suas casas decimais, toma-se apenas a parte inteira, porém não fazendo aproximação para o número inteiro mais próximo.

$4^{a}$ - Se uma UPA, em decorrência de seu "tamanho estimado" $D_{i}$, for selecionado $t$ vezes $(t>1)$, na segunda etapa, aplica-se amostragem sistemática com fração de amostragem $\frac{t b}{D_{i}}$, portanto com intervalo igual a $\frac{1}{t}$ daquele que seria aplicado se a UPA fosse selecionada apenas uma vez.

$5^{a}$ - Instruções para o percurso de cada UPA sorteada.

No percurso de cada UPA sorteada, procede-se da forma a seguir descrita.

a) Na aplicação da amostragem sistemática, o percurso da UPA deve ser completo, de forma que a UPA toda seja percorrida.

b) Em todos os domicílios da UPA deve ser verificada a existência de crianças.

c) Os domicílios fechados e portanto em relação aos quais não se sabe da existência ou não de crianças, devem ser registrados para formar um estrato a parte.

d) Os domicílios onde se constata que residem crianças devem ser registrados e numerados de forma que, com o percurso da UPA, vai sendo elaborada a lista dos domicílios com crianças. 
e) Na feitura dessa lista, quando é registrado um domicílio que, na numeração, recebe um número que é um dos "números selecionados", esse domicílio deve ser entrevistado, pois é um domicilio da amostra.

f) Faz-se uma segunda tentativa para obter informação sobre a existência de crianças, nos domicílios fechados, procurando mudar o horário de averiguação. Desses domicílios, aqueles que tiverem crianças devem ir recebendo numeração em sequêencia àquela dos domicílios com crianças, não fechados na primeira tentativa, e assim ficam sujeitos à amostragem sistemática que estava sendo aplicada a essa UPA.

Recomenda-se fazer mais duas tentativas com esse propósito.

g) Nos domicílios com crianças sorteadas, se a entrevista foi recusada ou houve algum impedimento para a sua realização, recomenda-se fazer mais até três retornos, procurando obter a informação.

$6^{a}-$ Seja:

$$
\bar{x}=\frac{\sum_{j=1}^{30} x_{j}}{30}
$$

$x_{j}$ número de crianças selecionadas na UPA $j$ da amostra.

O coeficiente de variação de $\bar{x}$ não deve superar $10 \%$, mas sendo inferior a $20 \%$ ainda é tolerável. Se esse coeficiente não atender a esse requisito, o pesquisador deve estar alertado de que o vício poderá ser não desprezível em relação ao erro padrão desse estimador e, além disso, uma das condições para a validade da fórmula que dá a variância do estimador, não estará sendo atendida. Nessa hipótese, recomenda-se para levantamentos futuros, em áreas análogas, verificar qual o número de UPA's a serem selecionadas na primeira etapa, a fim de que aquele requisito seja atendido.

$7^{a}$ - Se em uma UPA selecionada, o número de domicílios com crianças, existentes no período do levantamento $D_{c i}^{\prime}$, for menor que o intervalo $\frac{D_{i}}{b}$, pode ocorrer que o primeiro número selecionado seja maior que $D_{c i}^{\prime}$, resultando então que nenhum domicílio com criança seja selecionado nessa UPA. Com o intuito de que essa UPA, selecionada na primeira etapa, fique representada na amostra de 
domicílios com crianças, poderemos diminuir o intervalo da amostragem sistemática da segunda etapa, de forma que um ou mais daqueles domicílios sejam sorteados. Se entretanto o intervalo que era para ser aplicado, for substituido por um intervalo menor, a probabilidade de seleção dos domicílios com crianças dessa UPA ficará aumentada e este fato deve ser levado em conta ao ser feita a estimação da cobertura vacinal e, para tal, os valores encontrados na subamostra dessa UPA receberão um peso adequado, o qual será menor que o atribuido aos valores das subamostras das demais UPA's, para as quais não houve alteração do intervalo $\frac{D_{i}}{b}$ correspondente. Observações análogas fazemos em relação à estimação da variância do estimador.

Todas as crianças do grupo etário estudado, residentes nos domicilios sorteados, devem ser consideradas da amostra. De acordo com este método, todas as crianças da área de interesse têm igual probabilidade de pertencer à amostra, probabilidade esta dada pelo valor da fração amostral $f$, e, portanto a amostragem é eqüiprobabilística". 


\section{Material e Método}

Com a finalidade de comparar os três métodos de amostragem foram construidas quatro populaçôes hipotéticas em microcomputador PC-XT utilizando-se linguagens de programação BASIC e PASCAL.

\subsection{Desenho da Simulação}

As populacões foram constituídas a partir da combinação de conglomerados simulados separadamente. Foram gerados 334 conglomerados com características diferentes, ou seja, considerou-se no desenho o tamanho do conglomerado, a densidade populacional intradomiciliar, a densidade intradomiciliar de crianças, a cobertura vacinal e a taxa de resposta.

Para se fixar o campo de variacão dos valores para cada um dos parâmetros anteriormente descritos utilizaram-se informacões do censo de 1980 publicadas pela FIBGE $^{2}$ e por pesquisas que realizaram levantamentos de dados análogos para o caso das taxas de respostas.

- Tamanho do Conglomerado: Os conglomerados foram construídos baseados nos setores censitários. Um setor censitário é uma área geográfica perfeitamente definida que possui em média 300 domicílios. Estabele ceram-se quatro tamanhos de conglomerados conforme mostra a Tabela 4.1 .

Tabela 4.1 Distribuição dos Conglomerados segundo o número total de domicilios

\begin{tabular}{cc}
\hline $\begin{array}{c}\text { No. total } \\
\text { domicílio }\end{array}$ & Frequências \\
\hline 200 & 75 \\
300 & 107 \\
450 & 88 \\
600 & 64 \\
\hline Total & 334 \\
\hline
\end{tabular}


- Densidade Populacional Intradomiciliar: A densidade populacional intradomiciliar, que é a razão entre o número total de indivíduos residentes em uma área geográfica delimitada e o número total de domicílios desta mesma área, foi definida considerando-se os limites máximo e mínimo encontrados para o Brasil em 1980. Desta forma os conglomerados foram distribuídos conforme a Tabela 4.2.

Tabela 4.2 Distribuição dos Conglomerados segundo a densidade intradomiciliar

\begin{tabular}{cc}
\hline $\begin{array}{c}\text { No. } \\
\text { ind. }\end{array}$ & Frequências \\
\hline domic. & \\
\hline 3,5 & 39 \\
4,0 & 111 \\
4,5 & 45 \\
5,0 & 35 \\
5,5 & 48 \\
6,0 & 56 \\
\hline Total & 334 \\
\hline
\end{tabular}

- Densidade Intradomiciliar de Criancas: A densidade intradomiciliar de crianças com idade entre 12 e 24 meses (razão entre o número total de crianças residentes em uma área geográfica delimitada e o número total de domicílios desta mesma área) também foi estabelecida em função dos limites máximo e mínimo encontrados para o Brasil em 1980. A Tabela 4.3 apresenta estes valores e o número correspondente de conglomerados. 
Tabela 4.3 Distribuição dos Conglomerados segundo a densidade intradomiciliar de crianças

\begin{tabular}{cc}
\hline $\begin{array}{c}\text { No. } \\
\frac{\text { criancas }}{\text { domic }}\end{array}$ & Frequências \\
\hline 0,10 & 39 \\
0,11 & 57 \\
0,12 & 54 \\
0,13 & 45 \\
0,14 & 35 \\
0,15 & 48 \\
0,16 & 56 \\
\hline Total & 334 \\
\hline
\end{tabular}

- Cobertura Vacinal: A cobertura vacinal para cada conglomerado populacional é dada pelo número de crianças vacinadas do grupo etário em estudo dividido pelo número total de crianças deste mesmo grupo. A Tabela 4.4 apresenta os conglomerados de acordo com as suas coberturas vacinais planejadas para a simulação. 
Tabela 4.4 Distribuição dos Conglomerados segundo a cobertura vacinal planejada

\begin{tabular}{cc}
\hline $\begin{array}{c}\text { Cobertura } \\
\text { vacinal }\end{array}$ & Frequências \\
\hline 0,10 & 24 \\
0,20 & 9 \\
0,30 & 1 \\
0,40 & 24 \\
0,50 & 33 \\
0,60 & 59 \\
0,70 & 41 \\
0,80 & 35 \\
0,90 & 38 \\
0,98 & 70 \\
\hline Total & 334 \\
\hline
\end{tabular}

- Taxa de Resposta: A taxa de resposta de um levantamento é definida como a razão entre o número de observações (que podem ser respostas para um questionário ou algum outro tipo de medida) obtidas de elementos elegiveis e o número total de elementos elegiveis na amostra. Em levantamentos que utilizam entrevistas domiciliares, a ausência de resposta geralmente ocorre por alguma destas razões: recusa do entrevistado em responder, impossibilidade de contactar o indivíduo selecionado por estar ausente no domicilio, incapacidade do indivíduo para responder por motivo de doença, ou ainda, questionários já preenchidos podem ser perdidos em algum momento do processo.

A preocupacão com a taxa de resposta é devida à possibilidade do grupo de não respondentes ser diferente daquele que respondeu em relação às variáveis de interesse no estudo e, desta maneira, estimativas baseadas apenas nos respondentes serão viciadas para os parâmetros da população global. 
Observando-se levantamentos de dados realizados através de inquéritos domiciliares realizados no Brasil verifica-se que as taxas de resposta ficam em torno de $90 \%$. Kish considera que taxas de resposta de até $80 \%$ são aceitáveis, ou seja, o vício das estimativas não chegam a comprometer os resultados da pesquisa. Em vista destes fatos as taxas de resposta variaram entre 0,80 e 0,95 conforme mostra a Tabela 4.5.

\section{Tabela 4.5 Distribuição dos Conglomerados segundo a taxa de resposta}

\begin{tabular}{cc}
\hline $\begin{array}{c}\text { Taxa de } \\
\text { resposta }\end{array}$ & Frequências \\
\hline 0,95 & 110 \\
0,90 & 104 \\
0,85 & 83 \\
0,80 & 37 \\
\hline Total & 334 \\
\hline
\end{tabular}

Com estes conglomerados foram constituidas quatro populaçôes hipotéticas comparáveis entre si, ou seja, a única diferença entre elas foram as coberturas vacinais. As Figuras 4.1 a 4.4 e os Quadros 4.1 a 4.4 representam estas populações.

A ordenação dos setores para a composição dos sistemas de referência apresentadas nestes Quadros foi aleatória (sorteio com Tabela de Números Aleatórios).

Observando-se as figuras que representam as populações verifica-se que elas foram constituidas de tal forma que a cobertura vacinal de cada uma delas refletisse situações reais e ao mesmo tempo condições nas quais a discriminação do comportamento dos métodos de amostragem em estudo fosse mais fácil. 
QUADRO 4.1 - POPULACAO PLAHENADA 1

\begin{tabular}{|c|c|c|c|c|c|c|c|}
\hline $\begin{array}{l}\text { NOAERO DE } \\
\text { ORDEI }\end{array}$ & $\begin{array}{l}\text { DUMERO DA } \\
\text { OPA }\end{array}$ & $\begin{array}{l}\text { RUAERO DE } \\
\text { DOHICILIOS }\end{array}$ & $\begin{array}{l}\text { TAXA DE } \\
\text { RESPOSTA }\end{array}$ & $\begin{array}{l}\text { DENS. INTRA. } \\
\text { DE CRIANCAS }\end{array}$ & $\begin{array}{l}\text { COBERTURA } \\
\text { VACIRAL }\end{array}$ & $\begin{array}{l}\text { POPULACAO } \\
\text { DA UPA }\end{array}$ & $\begin{array}{l}\text { POPULACAO } \\
\text { ACUMULADA }\end{array}$ \\
\hline 1 & $\begin{array}{l}53 \\
83\end{array}$ & $\begin{array}{l}450 \\
450\end{array}$ & $\begin{array}{l}0,95 \\
0.90\end{array}$ & $\begin{array}{l}0,12 \\
0,11\end{array}$ & $\begin{array}{l}0,70 \\
0,80\end{array}$ & $\begin{array}{l}1800 \\
1800\end{array}$ & $\begin{array}{l}1800 \\
3600\end{array}$ \\
\hline 3 & 3 & 300 & 0,80 & 0,16 & 0,60 & 1800 & 5400 \\
\hline 4 & 70 & 300 & 0,90 & 0,11 & 0,70 & 1200 & 6600 \\
\hline 5 & 27 & 200 & 0,90 & 0,14 & 0,70 & 1000 & 7600 \\
\hline 6 & 26 & 200 & 0,90 & 0,14 & 0,70 & 1000 & 8600 \\
\hline 7 & 85 & 600 & 0,85 & 0,11 & 0,80 & 2400 & 11000 \\
\hline 8 & 97 & 450 & 0,85 & 0,10 & 0,90 & 1575 & 12575 \\
\hline 9 & 17 & 300 & 0,90 & 0,15 & 0,60 & 1650 & 14225 \\
\hline 10 & 77 & 200 & 0,95 & 0,11 & 0,80 & 800 & 15025 \\
\hline 11 & 78 & 200 & 0,95 & 0,11 & 0,80 & 800 & 15825 \\
\hline 12 & 95 & 300 & 0,80 & 0,10 & 0,90 & 1050 & 16875 \\
\hline 13 & $B O$ & 300 & 0,90 & 0,11 & 0,80 & 1200 & 18075 \\
\hline 18 & 74 & 600 & 0,85 & 0,11 & 0,70 & 2400 & 20475 \\
\hline 15 & 30 & 450 & 0,80 & 0,14 & 0,70 & 2250 & 22725 \\
\hline 16 & 48 & 200 & 0,95 & 0,15 & 0,70 & 1100 & 23825 \\
\hline 17 & 33 & 200 & 0,85 & 0,13 & 0,70 & 900 & 24725 \\
\hline 18 & 13 & 600 & 0,95 & 0,16 & 0,60 & 3600 & 28325 \\
\hline 19 & 71 & 300 & 0,95 & 0,11 & 0,70 & 1200 & 29525 \\
\hline 20 & 39 & 450 & 0,85 & 0,13 & 0,70 & 2025 & 31550 \\
\hline 21 & 96 & 300 & 0,95 & 0,10 & 0,90 & 1050 & 32600 \\
\hline 22 & 15 & 200 & 0,85 & 0,15 & 0,60 & 1100 & 33700 \\
\hline 23 & 7 & 450 & 0,90 & 0,16 & 0,60 & 2700 & 36400 \\
\hline 24 & 98 & 450 & 0,90 & 0,10 & 0,90 & 1575 & 37975 \\
\hline 25 & 41 & 600 & 0,95 & 0,13 & 0,70 & 2700 & 40675 \\
\hline 26 & 52 & 450 & 0,85 & 0,12 & 0,70 & 1800 & 42475 \\
\hline 27 & 84 & 450 & 0,90 & 0,11 & 0,80 & 1800 & 44275 \\
\hline 28 & 68 & 200 & 0,95 & 0,11 & 0,70 & 800 & 45075 \\
\hline 29 & 23 & 450 & 0,95 & 0,15 & 0,60 & 2475 & 47550 \\
\hline 30 & 92 & 600 & 0,90 & 0,10 & 0,80 & 2100 & 49650 \\
\hline 31 & 58 & 200 & 0,90 & 0,12 & 0,80 & 800 & 50450 \\
\hline 32 & 36 & 300 & 0,95 & 0,13 & 0,70 & 1350 & 51800 \\
\hline 33 & 32 & 600 & 0,90 & 0,14 & 0,70 & 3000 & 54800 \\
\hline 34 & 18 & 300 & 0,85 & 0,15 & 0,60 & 1650 & 56450 \\
\hline 35 & 29 & 300 & 0,85 & 0,14 & 0,70 & 1500 & 57950 \\
\hline 36 & 34 & 300 & 0,85 & 0,13 & 0,70 & 1350 & 59300 \\
\hline 37 & 6 & 300 & 0,95 & 0,16 & 0,60 & 1800 & 61100 \\
\hline 38 & 69 & 300 & 0,90 & 0,11 & 0,70 & 1200 & 62300 \\
\hline 39 & 42 & 200 & 0,90 & 0,13 & 0,80 & 900 & 63200 \\
\hline 40 & 63 & 450 & 0,80 & 0,12 & 0,80 & 1800 & 65000 \\
\hline 41 & 89 & 450 & 0,90 & 0,10 & 0,80 & 1575 & 66575 \\
\hline 42 & 65 & 450 & 0,95 & 0,12 & 0,80 & 1800 & 68375 \\
\hline 43 & 5 & 300 & 0,95 & 0,16 & 0,60 & 1800 & 70175 \\
\hline 44 & 25 & 200 & 0,90 & 0,14 & 0,70 & 1000 & 71175 \\
\hline 45 & 82 & 450 & 0,85 & 0,11 & 0,80 & 1800 & 72975 \\
\hline 46 & 61 & 300 & 0,95 & 0,12 & 0,80 & 1200 & 74175 \\
\hline 47 & 57 & 200 & 0,90 & 0,12 & 0,80 & 800 & 74975 \\
\hline 48 & 62 & 450 & 0,80 & 0,12 & 0,80 & 1800 & 76775 \\
\hline 49 & 46 & 450 & 0,95 & 0,13 & 0,80 & 2025 & 78800 \\
\hline 50 & 73 & 450 & 0,90 & 0,11 & 0,70 & 1800 & 80600 \\
\hline
\end{tabular}


QUADRO 4.1 - POPULACAO PLAMEJADA 1 (Conclusao)

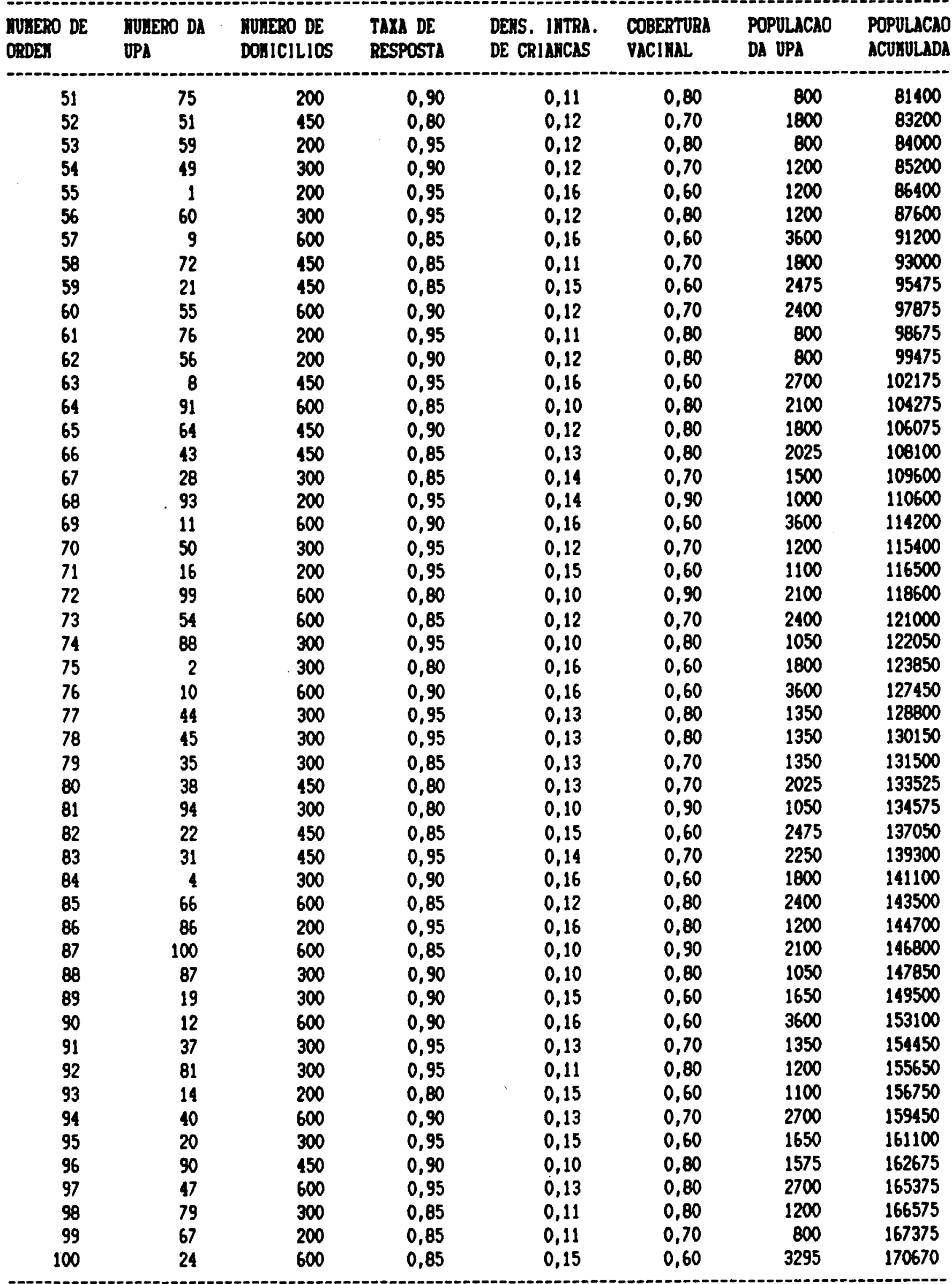


QUADRO 4.2 - POPULACAO PLAMEJADA 2

\begin{tabular}{|c|c|c|c|c|c|c|c|}
\hline $\begin{array}{l}\text { NOHESO DE } \\
\text { ORDED }\end{array}$ & $\begin{array}{l}\text { ROAERO DA } \\
\text { OPA }\end{array}$ & $\begin{array}{l}\text { ROAERO DE } \\
\text { DOAICILIOS }\end{array}$ & $\begin{array}{l}\text { TAXA DE } \\
\text { RESPOSTA }\end{array}$ & $\begin{array}{l}\text { DEIS. INTRA. } \\
\text { DE CRIANCAS }\end{array}$ & $\begin{array}{l}\text { COBERTURA } \\
\text { VACIMAL }\end{array}$ & $\begin{array}{l}\text { POPULACAO } \\
\text { DA UPA }\end{array}$ & $\begin{array}{l}\text { POPOLACAO } \\
\text { ACUAULADA }\end{array}$ \\
\hline 1 & 53 & 450 & 0,95 & 0,12 & 0,70 & 1800 & 1800 \\
\hline 2 & 83 & 450 & 0,90 & 0,11 & 0,80 & 1800 & 3600 \\
\hline 3 & 3 & 300 & 0,80 & 0,16 & 0,10 & 1800 & 5400 \\
\hline 4 & 70 & 300 & 0,90 & 0,11 & 0,70 & 1200 & 6600 \\
\hline 5 & 27 & 200 & 0,90 & 0,14 & 0,20 & 1000 & 7600 \\
\hline 6 & 26 & 200 & 0,90 & 0,14 & 0,20 & 1000 & 8600 \\
\hline 7 & 85 & 600 & 0,85 & 0,11 & 0,80 & 2400 & 11000 \\
\hline 8 & 97 & 450 & 0,85 & 0,10 & 0,90 & 1575 & 12575 \\
\hline 9 & 17 & 300 & 0,90 & 0,15 & 0,10 & 1650 & 14225 \\
\hline 10 & 77 & 200 & 0,95 & 0,11 & 0,80 & 800 & 15025 \\
\hline 11 & 78 & 200 & 0,95 & 0,11 & 0,80 & 800 & 15825 \\
\hline 12 & 95 & 300 & 0,80 & 0,10 & 0,90 & 1050 & 16875 \\
\hline 13 & 80 & 300 & 0,90 & 0,11 & 0,80 & 1200 & 18075 \\
\hline 14 & 74 & 600 & 0,85 & 0,11 & 0,70 & 2400 & 20475 \\
\hline 15 & 30 & 450 & 0,80 & 0,14 & 0,20 & 2250 & 22725 \\
\hline 16 & 48 & 200 & 0,95 & 0,15 & 0,20 & 1100 & 23825 \\
\hline 17 & 33 & 200 & 0,85 & 0,13 & 0,70 & 900 & 24725 \\
\hline 18 & 13 & 600 & 0,95 & 0,16 & 0,10 & 3600 & 28325 \\
\hline 19 & 71 & 300 & 0,95 & 0,11 & 0,70 & 1200 & 29525 \\
\hline 20 & 39 & 450 & 0,85 & 0,13 & 0,70 & 2025 & 31550 \\
\hline 21 & 96 & 300 & 0,95 & 0,10 & 0,90 & 1050 & 32600 \\
\hline 22 & 15 & 200 & 0,85 & 0,15 & 0,10 & 1100 & 33700 \\
\hline 23 & 7 & 450 & 0,90 & 0,16 & 0,10 & 2700 & 36400 \\
\hline 24 & 98 & 450 & 0,90 & 0,10 & 0,90 & 1575 & 37975 \\
\hline 25 & 41 & 600 & 0,95 & 0,13 & 0,70 & 2700 & 40675 \\
\hline 26 & 52 & 450 & 0,85 & 0,12 & 0,70 & 1800 & 42475 \\
\hline 27 & 84 & 450 & 0,90 & 0,11 & 0,80 & 1800 & $\$ 4275$ \\
\hline 28 & 68 & 200 & 0,95 & 0,11 & 0,70 & 800 & 45075 \\
\hline 29 & 23 & 450 & 0,95 & 0,15 & 0,10 & 2475 & 47550 \\
\hline 30 & 92 & 600 & 0,90 & 0,10 & 0,80 & 2100 & 49650 \\
\hline 31 & 58 & 200 & 0,90 & 0,12 & 0,80 & 800 & 50450 \\
\hline 32 & 36 & 300 & 0,95 & 0,13 & 0,70 & 1350 & 51800 \\
\hline 33 & 32 & 600 & 0,90 & 0,14 & 0,20 & 3000 & 54800 \\
\hline 34 & 18 & 300 & 0,85 & 0,15 & 0,10 & 1650 & 56450 \\
\hline 35 & 29 & 300 & 0,85 & 0,14 & 0,20 & 1500 & 57950 \\
\hline 36 & 34 & 300 & 0,85 & 0,13 & 0,70 & 1350 & 59300 \\
\hline 37 & 6 & 300 & 0,95 & 0,16 & 0,10 & 1800 & 61100 \\
\hline 38 & 69 & 300 & 0,90 & 0,11 & 0,70 & 1200 & 62300 \\
\hline 39 & 42 & 200 & 0,90 & 0,13 & 0,80 & 900 & 63200 \\
\hline 40 & 63 & 450 & 0,80 & 0,12 & 0,80 & 1800 & 65000 \\
\hline 41 & 89 & 450 & 0,90 & 0,10 & 0,80 & 1575 & 66575 \\
\hline 42 & 65 & 450 & 0,95 & 0,12 & 0,80 & 1800 & 68375 \\
\hline 43 & 5 & 300 & 0,95 & 0,16 & 0,10 & 1800 & 70175 \\
\hline 44 & 25 & 200 & 0,90 & 0,14 & 0,20 & 1000 & 71175 \\
\hline 45 & 82 & 450 & 0,85 & 0,11 & 0,80 & 1800 & 72975 \\
\hline 46 & 61 & 300 & 0,95 & 0,12 & 0,80 & 1200 & 74175 \\
\hline 47 & 57 & 200 & 0,90 & 0,12 & 0,80 & 800 & 74975 \\
\hline 48 & 62 & 450 & 0,80 & 0,12 & 0,80 & 1800 & 76775 \\
\hline 49 & 46 & 450 & 0,95 & 0,13 & 0,80 & 2025 & 78800 \\
\hline 50 & 73 & 450 & 0,90 & 0,11 & 0,70 & 1800 & 80600 \\
\hline
\end{tabular}


QUADRO 4.2 - POPULACAO PLAREJADA 2 (Conclusao)

\begin{tabular}{|c|c|c|c|c|c|c|c|}
\hline $\begin{array}{l}\text { WOIERO DE } \\
\text { ODDEX }\end{array}$ & $\begin{array}{l}\text { DOLIERO DA } \\
\text { OPA }\end{array}$ & $\begin{array}{l}\text { DUIERO DE } \\
\text { DOHICILIOS }\end{array}$ & $\begin{array}{l}\text { TAXA DE } \\
\text { RESPOSTA }\end{array}$ & $\begin{array}{l}\text { DEAS. INTRA. } \\
\text { DE CRIAMCAS }\end{array}$ & $\begin{array}{l}\text { COBERTTURA } \\
\text { VACIILLL }\end{array}$ & $\begin{array}{l}\text { POPOLACAO } \\
\text { DA UPA }\end{array}$ & $\begin{array}{l}\text { POPOLACAO } \\
\text { ACULULADA }\end{array}$ \\
\hline 51 & 75 & 200 & 0,90 & 0,11 & 0,80 & 800 & 81400 \\
\hline 52 & 51 & 450 & 0,80 & 0,12 & 0,70 & 1800 & 83200 \\
\hline 53 & 59 & 200 & 0,95 & 0,12 & 0,80 & 800 & 84000 \\
\hline 54 & 49 & 300 & 0,90 & 0,12 & 0,70 & 1200 & 85200 \\
\hline 55 & 1 & 200 & 0,95 & 0,16 & 0,10 & 1200 & 86400 \\
\hline 56 & 60 & 300 & 0,95 & 0,12 & 0,80 & 1200 & 87600 \\
\hline 57 & 9 & 600 & 0,85 & 0,16 & 0,10 & 3600 & 91200 \\
\hline 58 & 72 & 450 & 0,85 & 0,11 & 0,70 & 1800 & 93000 \\
\hline 59 & 21 & 450 & 0,85 & 0,15 & 0,10 & 2475 & 95475 \\
\hline 60 & 55 & 600 & 0,90 & 0,12 & 0,70 & 2400 & 97875 \\
\hline 61 & 76 & 200 & 0,95 & 0,11 & 0,00 & 800 & 98675 \\
\hline 62 & 56 & 200 & 0,90 & 0,12 & 0,80 & 800 & 99475 \\
\hline 63 & $B$ & 450 & 0,95 & 0,16 & 0,10 & 2700 & 102175 \\
\hline 64 & 91 & 600 & 0,85 & 0,10 & 0,80 & 2100 & 104275 \\
\hline 65 & 64 & 450 & 0,90 & 0,12 & 0,80 & 1800 & 106075 \\
\hline 66 & 43 & 450 & 0,85 & 0,13 & 0,80 & 2025 & 108100 \\
\hline 67 & 28 & 300 & 0,85 & 0,14 & 0,20 & 1500 & 109600 \\
\hline 68 & 93 & 200 & 0,95 & 0,14 & 0,90 & 1000 & 110600 \\
\hline 69 & 11 & 600 & 0,90 & 0,16 & 0,10 & 3600 & 114200 \\
\hline 70 & 50 & 300 & 0,95 & 0,12 & 0,70 & 1200 & 115400 \\
\hline 71 & 16 & 200 & 0,95 & 0,15 & 0,10 & 1100 & 116500 \\
\hline 72 & 99 & 600 & 0,80 & 0,10 & 0,90 & 2100 & 118600 \\
\hline 73 & 54 & 600 & 0,85 & 0,12 & 0,70 & 2400 & 121000 \\
\hline 74 & $B 8$ & 300 & 0,95 & 0,10 & 0,80 & 1050 & 122050 \\
\hline 75 & 2 & 300 & 0,80 & 0,16 & 0,10 & 1800 & 123850 \\
\hline 76 & 10 & 600 & 0,90 & 0,16 & 0,10 & 3600 & 127450 \\
\hline 77 & 44 & 300 & 0,95 & 0,13 & 0,80 & 1350 & 128800 \\
\hline 78 & 45 & 300 & 0,95 & 0,13 & 0,80 & 1350 & 130150 \\
\hline 79 & 35 & 300 & 0,85 & 0,13 & 0,70 & 1350 & 131500 \\
\hline 80 & 38 & 450 & 0,80 & 0,13 & 0,70 & 2025 & 133525 \\
\hline 81 & 94 & 300 & 0,80 & 0,10 & 0,90 & 1050 & 134575 \\
\hline 82 & 22 & 450 & 0,85 & 0,15 & 0,10 & 2475 & 137050 \\
\hline 83 & 31 & 450 & 0,95 & 0,14 & 0,20 & 2250 & 139300 \\
\hline 84 & 4 & 300 & 0,90 & 0,16 & 0,10 & 1800 & 141100 \\
\hline 85 & 66 & 600 & 0,85 & 0,12 & 0,80 & 2400 & 143500 \\
\hline 86 & 86 & 200 & 0,95 & 0,16 & 0,30 & 1200 & 144700 \\
\hline 87 & 100 & 600 & 0,85 & 0,10 & 0,90 & 2100 & 146800 \\
\hline 88 & 87 & 300 & 0,90 & 0,10 & 0,80 & 1050 & 147850 \\
\hline 89 & 19 & 300 & 0,90 & 0,15 & 0,10 & 1650 & 149500 \\
\hline 90 & 12 & 600 & 0,90 & 0,16 & 0,10 & 3600 & 153100 \\
\hline 91 & 37 & 300 & 0,95 & 0,13 & 0,70 & 1350 & 154450 \\
\hline 92 & 81 & 300 & 0,95 & 0,11 & 0,80 & 1200 & 155650 \\
\hline 93 & 14 & 200 & 0,80 & 0,15 & 0,10 & 1100 & 156750 \\
\hline 94 & 40 & 600 & 0,90 & 0,13 & 0,70 & 2700 & 159450 \\
\hline 95 & 20 & 300 & 0,95 & 0,15 & 0,10 & 1650 & 161100 \\
\hline 96 & 90 & 450 & 0,90 & 0,10 & 0,80 & 1575 & 162675 \\
\hline 97 & 47 & 600 & 0,95 & 0,13 & 0,80 & 2700 & 165375 \\
\hline 98 & 79 & 300 & 0,85 & 0,11 & 0,80 & 1200 & 166575 \\
\hline 99 & 67 & 200 & 0,85 & 0,11 & 0,70 & 800 & 167375 \\
\hline 100 & 24 & 600 & 0,85 & 0,15 & 0,10 & 3295 & 170670 \\
\hline
\end{tabular}


QUADRO 4.3 - POPULACAO PLAREJADA 3

\begin{tabular}{|c|c|c|c|c|c|c|c|}
\hline $\begin{array}{l}\text { ROWERO DE } \\
\text { ORDER }\end{array}$ & $\begin{array}{l}\text { MUHERO DA } \\
\text { UPA }\end{array}$ & $\begin{array}{l}\text { RUIERO DE } \\
\text { DOHICILIOS }\end{array}$ & $\begin{array}{l}\text { TAXA DE } \\
\text { RESPOSTA }\end{array}$ & $\begin{array}{l}\text { DEAS. IITRA. } \\
\text { DE CRIAMCAS }\end{array}$ & $\begin{array}{l}\text { COBERTURA } \\
\text { VACIKAL }\end{array}$ & $\begin{array}{l}\text { POPULACAO } \\
\text { DA OPA }\end{array}$ & $\begin{array}{l}\text { POPULACAC } \\
\text { ACUAULADI }\end{array}$ \\
\hline 1 & 53 & 450 & 0,95 & 0,12 & 0,50 & 1800 & 1800 \\
\hline 2 & 83 & 450 & 0,90 & 0,11 & 0,60 & 1800 & 3600 \\
\hline 3 & 3 & 300 & 0,80 & 0,16 & 0,40 & 1800 & 5400 \\
\hline 4 & 70 & 300 & 0,90 & 0,11 & 0,50 & 1200 & 6600 \\
\hline 5 & 27 & 200 & 0,90 & 0,14 & 0,50 & 1000 & 7600 \\
\hline 6 & 26 & 200 & 0,90 & 0,14 & 0,50 & 1000 & 8600 \\
\hline 7 & 85 & 600 & 0,85 & 0,11 & 0,60 & 2400 & 11000 \\
\hline B & 97 & 450 & 0,85 & 0,10 & 0,70 & 1575 & 12575 \\
\hline 9 & 17 & 300 & 0,90 & 0,15 & 0,40 & 1650 & 14225 \\
\hline 10 & 77 & 200 & 0,95 & 0,11 & 0,60 & 800 & 15025 \\
\hline 11 & 78 & 200 & 0,95 & 0,11 & 0,60 & 800 & 15825 \\
\hline 12 & 95 & 300 & 0,80 & 0,10 & 0,70 & 1050 & 16875 \\
\hline 13 & 80 & 300 & 0,90 & 0,11 & 0,60 & 1200 & 18075 \\
\hline 14 & 74 & 600 & 0,85 & 0,11 & 0,50 & 2400 & 20475 \\
\hline 15 & 30 & 450 & 0,80 & 0,14 & 0,50 & 2250 & 22725 \\
\hline 16 & 48 & 200 & 0,95 & 0,15 & 0,50 & 1100 & 23825 \\
\hline 17 & 33 & 200 & 0,85 & 0,13 & 0,50 & 900 & 24725 \\
\hline 18 & 13 & 600 & 0,95 & 0,16 & 0,40 & 3600 & 28325 \\
\hline 19 & 71 & 300 & 0,95 & 0,11 & 0,50 & 1200 & 29525 \\
\hline 20 & 39 & 450 & 0,85 & 0,13 & 0,50 & 2025 & 31550 \\
\hline 21 & 96 & 300 & 0,95 & 0,10 & 0,70 & 1050 & 32600 \\
\hline 22 & 15 & 200 & 0,85 & 0,15 & 0,40 & 1100 & 33700 \\
\hline 23 & 7 & 450 & 0,90 & 0,16 & 0,40 & 2700 & 36400 \\
\hline 24 & 98 & 450 & 0,90 & 0,10 & 0,70 & 1575 & 37975 \\
\hline 25 & 41 & 600 & 0,95 & 0,13 & 0,50 & 2700 & 40675 \\
\hline 26 & 52 & 450 & 0,85 & 0,12 & 0,50 & 1800 & 42475 \\
\hline 27 & 84 & 450 & 0,90 & 0,11 & 0,60 & 1800 & 44275 \\
\hline 28 & 68 & 200 & 0,95 & 0,11 & 0,50 & 800 & 45075 \\
\hline 29 & 23 & 450 & 0,95 & 0,15 & 0,40 & 2475 & 47550 \\
\hline 30 & 92 & 600 & 0,90 & 0,10 & 0,60 & 2100 & 49650 \\
\hline 31 & 58 & 200 & 0,90 & 0,12 & 0,60 & 800 & 50450 \\
\hline 32 & 36 & 300 & 0,95 & 0,13 & 0,50 & 1350 & 51800 \\
\hline 33 & 32 & 600 & 0,90 & 0,14 & 0,50 & 3000 & 54800 \\
\hline 34 & 18 & 300 & 0,85 & 0,15 & 0,40 & 1650 & 56450 \\
\hline 35 & 29 & 300 & 0,85 & 0,14 & 0,50 & 1500 & 57950 \\
\hline 36 & 34 & 300 & 0,85 & 0,13 & 0,50 & 1350 & 59300 \\
\hline 37 & 6 & 300 & 0,95 & 0,16 & 0,40 & 1800 & 61100 \\
\hline 38 & 69 & 300 & 0,90 & 0,11 & 0,50 & 1200 & 62300 \\
\hline 39 & 42 & 200 & 0,90 & 0,13 & 0,60 & 900 & 63200 \\
\hline 40 & 63 & 450 & 0,80 & 0,12 & 0,60 & 1800 & 65000 \\
\hline 41 & 89 & 450 & 0,90 & 0,10 & 0,60 & 1575 & 66575 \\
\hline 42 & 65 & 450 & 0,95 & 0,12 & 0,60 & 1800 & 68375 \\
\hline 43 & 5 & 300 & 0,95 & 0,16 & 0,40 & 1800 & 70175 \\
\hline 44 & 25 & 200 & 0,90 & 0,14 & 0,50 & 1000 & 71175 \\
\hline 45 & 82 & 450 & 0,85 & 0,11 & 0,60 & 1800 & 72975 \\
\hline 46 & 61 & 300 & 0,95 & 0,12 & 0,60 & 1200 & 74175 \\
\hline 47 & 57 & 200 & 0,90 & 0,12 & 0,60 & 800 & 74975 \\
\hline 48 & 62 & 450 & 0,80 & 0,12 & 0,60 & 1800 & 76775 \\
\hline 19 & 46 & 450 & 0,95 & 0,13 & 0,60 & 2025 & 78800 \\
\hline 50 & 73 & 450 & 0,90 & 0,11 & 0,50 & 1800 & 80600 \\
\hline
\end{tabular}


QUADRO 4.3 - POPULACAO PLANEJADA 3 (Conclusao)

\begin{tabular}{|c|c|c|c|c|c|c|c|}
\hline $\begin{array}{l}\text { IOAERO DE } \\
\text { ORDEM }\end{array}$ & $\begin{array}{l}\text { MUAERO DA } \\
\text { UPA }\end{array}$ & $\begin{array}{l}\text { MULERO DE } \\
\text { DOHICILIOS }\end{array}$ & $\begin{array}{l}\text { TAXA DE } \\
\text { RESPOSTA }\end{array}$ & $\begin{array}{l}\text { DEAS. INTRA. } \\
\text { DE CRIANCAS }\end{array}$ & $\begin{array}{l}\text { COBERTURA } \\
\text { VACIIALL }\end{array}$ & $\begin{array}{l}\text { POPOLACAO } \\
\text { DA UPA }\end{array}$ & $\begin{array}{l}\text { POPULLACAO } \\
\text { ACUSULADA }\end{array}$ \\
\hline 51 & 75 & 200 & 0,90 & 0,11 & 0,60 & 800 & 81400 \\
\hline 52 & 51 & 450 & 0,80 & 0,12 & 0,50 & 1800 & 83200 \\
\hline 53 & 59 & 200 & 0,95 & 0,12 & 0,60 & 800 & 84000 \\
\hline 54 & 49 & 300 & 0,90 & 0,12 & 0,50 & 1200 & 85200 \\
\hline 55 & 1 & 200 & 0,95 & 0,16 & 0,40 & 1200 & 86400 \\
\hline 56 & 60 & 300 & 0,95 & 0,12 & 0,60 & 1200 & 87600 \\
\hline 57 & 9 & 600 & 0,85 & 0,16 & 0,40 & 3600 & 91200 \\
\hline 58 & 72 & 450 & 0,85 & 0,11 & 0,50 & 1800 & 93000 \\
\hline $\begin{array}{l}59 \\
60\end{array}$ & $\begin{array}{l}21 \\
55\end{array}$ & $\begin{array}{l}450 \\
600\end{array}$ & $\begin{array}{l}0,85 \\
0.90\end{array}$ & $\begin{array}{l}0,15 \\
0,12\end{array}$ & $\begin{array}{l}0,40 \\
0.50\end{array}$ & $\begin{array}{l}2475 \\
2400\end{array}$ & $\begin{array}{l}95475 \\
97875\end{array}$ \\
\hline 61 & 76 & 200 & 0,95 & 0,11 & 0,60 & 800 & 98675 \\
\hline 62 & 56 & 200 & 0,90 & 0,12 & 0,60 & 800 & 99475 \\
\hline 63 & 8 & 450 & 0,95 & 0,16 & 0,40 & 2700 & 102175 \\
\hline 64 & 91 & 600 & 0,85 & 0,10 & 0,60 & 2100 & 104275 \\
\hline 65 & 64 & 450 & 0,90 & 0,12 & 0,60 & 1800 & 106075 \\
\hline 66 & 43 & 450 & 0,85 & 0,13 & 0,60 & 2025 & 108100 \\
\hline 67 & 28 & 300 & 0,85 & 0,14 & 0,50 & 1500 & 109600 \\
\hline 68 & 93 & 200 & 0,95 & 0,14 & 0,70 & 1000 & 110600 \\
\hline 69 & 11 & 600 & 0,90 & 0,16 & 0,40 & 3600 & 114200 \\
\hline 70 & 50 & 300 & 0,95 & 0,12 & 0,50 & 1200 & 115400 \\
\hline 71 & 16 & 200 & 0,95 & 0,15 & 0,10 & 1100 & 116500 \\
\hline 72 & 99 & 600 & 0,80 & 0,10 & 0,70 & 2100 & 118600 \\
\hline 73 & 54 & 600 & 0,85 & 0,12 & 0,50 & 2400 & 121000 \\
\hline 74 & $B 8$ & 300 & 0,95 & 0,10 & 0,60 & 1050 & 122050 \\
\hline 75 & 2 & 300 & 0,80 & 0,16 & 0,40 & 1800 & 123850 \\
\hline 76 & 10 & 600 & 0,90 & 0,16 & 0,40 & 3600 & 127450 \\
\hline 77 & 44 & 300 & 0,95 & 0,13 & 0,60 & 1350 & 128800 \\
\hline 78 & 45 & 300 & 0,95 & 0,13 & 0,60 & 1350 & 130150 \\
\hline 79 & 35 & 300 & 0,85 & 0,13 & 0,50 & 1350 & 131500 \\
\hline$B O$ & 38 & 450 & 0,80 & 0,13 & 0,50 & 2025 & 133525 \\
\hline$B 1$ & 94 & 300 & 0,80 & 0,10 & 0,70 & 1050 & 134575 \\
\hline 82 & 22 & 450 & 0,85 & 0,15 & 0,40 & 2475 & 137050 \\
\hline 83 & 31 & 450 & 0,95 & 0,14 & 0,50 & 2250 & 139300 \\
\hline 84 & 4 & 300 & 0,90 & 0,16 & 0,40 & 1800 & 141100 \\
\hline 85 & 66 & 600 & 0,85 & 0,12 & 0,60 & 2400 & 143500 \\
\hline 86 & 86 & 200 & 0,95 & 0,16 & 0,60 & 1200 & 144700 \\
\hline 87 & 100 & 600 & 0,85 & 0,10 & 0,70 & 2100 & 146800 \\
\hline 88 & 87 & 300 & 0,90 & 0,10 & 0,60 & 1050 & 147850 \\
\hline 89 & 19 & 300 & 0,90 & 0,15 & 0,40 & 1650 & 149500 \\
\hline 90 & 12 & 600 & 0,90 & 0,16 & 0,40 & 3600 & 153100 \\
\hline 91 & 37 & 300 & 0,95 & 0,13 & 0,50 & 1350 & 154450 \\
\hline 92 & 81 & 300 & 0,95 & 0,11 & 0,60 & 1200 & 155650 \\
\hline 93 & 14 & 200 & 0,80 & 0,15 & 0,40 & 1100 & 156750 \\
\hline 94 & 40 & 600 & 0,90 & 0,13 & 0,50 & 2700 & 159450 \\
\hline 95 & 20 & 300 & 0,95 & 0,15 & 0,10 & 1650 & 161100 \\
\hline 96 & 90 & 450 & 0,90 & 0,10 & 0,60 & 1575 & 162675 \\
\hline 97 & 47 & 600 & 0,95 & 0,13 & 0,60 & 2700 & 165375 \\
\hline 98 & 79 & 300 & 0,85 & 0,11 & 0,60 & 1200 & 166575 \\
\hline 99 & 67 & 200 & 0,85 & 0,11 & 0,50 & 800 & 167375 \\
\hline 100 & 24 & 600 & 0,85 & 0,15 & 0,40 & 3295 & 170670 \\
\hline
\end{tabular}


QUADRO 4.4 - POPULACAO PLANEJADA 4

\begin{tabular}{|c|c|c|c|c|c|c|c|}
\hline $\begin{array}{l}\text { MOUEPO DE } \\
\text { ORDEN }\end{array}$ & $\begin{array}{l}\text { NUAERO DA } \\
\text { UPA }\end{array}$ & $\begin{array}{l}\text { MUSERO DE } \\
\text { DOHICILIOS }\end{array}$ & $\begin{array}{l}\text { TAXA DE } \\
\text { RESPOSTA }\end{array}$ & $\begin{array}{l}\text { DEAS. IMTRA, } \\
\text { DE CRIAMCAS }\end{array}$ & $\begin{array}{l}\text { COBERTURA } \\
\text { VACIMAL }\end{array}$ & $\begin{array}{l}\text { POPULACAO } \\
\text { DA UPA }\end{array}$ & $\begin{array}{l}\text { POPULACAO } \\
\text { ACUAULADA }\end{array}$ \\
\hline 1 & 53 & 450 & 0,95 & 0,12 & 0,90 & 1800 & 1800 \\
\hline 2 & 83 & 450 & 0,90 & 0,11 & 0,98 & 1800 & 3600 \\
\hline 3 & 3 & 300 & 0,80 & 0,16 & 0,98 & 1800 & 5400 \\
\hline 4 & 70 & 300 & 0,90 & 0,11 & 0,90 & 1200 & 6600 \\
\hline 5 & 27 & 200 & 0,90 & 0,14 & 0,98 & 1000 & 7600 \\
\hline 6 & 26 & 200 & 0,90 & 0,14 & 0,98 & 1000 & 8600 \\
\hline 7 & 85 & 600 & 0,85 & 0,11 & 0,98 & 2400 & 11000 \\
\hline 8 & 97 & 450 & 0,85 & 0,10 & 0,98 & 1575 & 12575 \\
\hline 9 & 17 & 300 & 0,90 & 0,15 & 0,98 & 1650 & 14225 \\
\hline 10 & 77 & 200 & 0,95 & 0,11 & 0,98 & 800 & 15025 \\
\hline 11 & 78 & 200 & 0,95 & 0,11 & 0,98 & 800 & 15825 \\
\hline 12 & 95 & 300 & 0,80 & 0,10 & 0,98 & 1050 & 16875 \\
\hline 13 & 80 & 300 & 0,90 & 0,11 & 0,98 & 1200 & 18075 \\
\hline 14 & 74 & 600 & 0,85 & 0,11 & 0,90 & 2400 & 20475 \\
\hline 15 & 30 & 450 & 0,80 & 0,14 & 0,98 & 2250 & 22725 \\
\hline 16 & 48 & 200 & 0,95 & 0,15 & 0,98 & 1100 & 23825 \\
\hline 17 & 33 & 200 & 0,85 & 0,13 & 0,90 & 900 & 24725 \\
\hline 18 & 13 & 600 & 0,95 & 0,16 & 0,98 & 3600 & 28325 \\
\hline 19 & 71 & 300 & 0,95 & 0,11 & 0,90 & 1200 & 29525 \\
\hline 20 & 39 & 450 & 0,85 & 0,13 & 0,90 & 2025 & 31550 \\
\hline 21 & 96 & 300 & $.0,95$ & 0,10 & 0,98 & 1050 & 32600 \\
\hline 22 & 15 & 200 & 0,85 & 0,15 & 0,98 & 1100 & 33700 \\
\hline 23 & 7 & 450 & 0,90 & 0,16 & 0,98 & 2700 & 36400 \\
\hline 24 & 98 & 450 & 0,90 & 0,10 & 0,98 & 1575 & 37975 \\
\hline 25 & 41 & 600 & 0,95 & 0,13 & 0,90 & 2700 & 40675 \\
\hline 26 & 52 & 450 & 0,85 & 0,12 & 0,90 & 1800 & 42475 \\
\hline 27 & 84 & 450 & 0,90 & 0,11 & 0,98 & 1800 & 44275 \\
\hline 28 & 68 & 200 & 0,95 & 0,11 & 0,90 & 800 & 45075 \\
\hline 29 & 23 & 450 & 0,95 & 0,15 & 0,98 & 2475 & 47550 \\
\hline 30 & 92 & 600 & 0,90 & 0,10 & 0,90 & 2100 & 49650 \\
\hline 31 & 58 & 200 & 0,90 & 0,12 & 0,98 & 800 & 50450 \\
\hline 32 & 36 & 300 & 0,95 & 0,13 & 0,90 & 1350 & 51800 \\
\hline 33 & 32 & 600 & 0,90 & 0,14 & 0,98 & 3000 & 54800 \\
\hline 34 & 18 & 300 & 0,85 & 0,15 & 0,98 & 1650 & 56450 \\
\hline 35 & 29 & 300 & 0,85 & 0,14 & 0,98 & 1500 & 57950 \\
\hline 36 & 34 & 300 & 0,85 & 0,13 & 0,90 & 1350 & 59300 \\
\hline 37 & 6 & 300 & 0,95 & 0,16 & 0,98 & 1800 & 61100 \\
\hline 38 & 69 & 300 & 0,90 & 0,11 & 0,90 & 1200 & 62300 \\
\hline 39 & 42 & 200 & 0,90 & 0,13 & 0,98 & 900 & 63200 \\
\hline 40 & 63 & 450 & 0,80 & 0,12 & 0,98 & 1800 & 65000 \\
\hline 11 & 89 & 450 & 0,90 & 0,10 & 0,90 & 1575 & 66575 \\
\hline 42 & 65 & 450 & 0,95 & 0,12 & 0,98 & 1800 & 68375 \\
\hline 43 & 5 & 300 & 0,95 & 0,16 & 0,98 & 1800 & 70175 \\
\hline 44 & 25 & 200 & 0,90 & 0,11 & 0,98 & 1000 & 71175 \\
\hline 45 & 82 & 450 & 0,85 & 0,11 & 0,98 & 1800 & 72975 \\
\hline 46 & 61 & 300 & 0,95 & 0,12 & 0,98 & 1200 & 74175 \\
\hline 47 & 57 & 200 & 0,90 & 0,12 & 0,98 & 800 & 74975 \\
\hline 48 & 62 & 450 & 0,80 & 0,12 & 0,98 & 1800 & 76775 \\
\hline 49 & 46 & 450 & 0,95 & 0,13 & 0,98 & 2025 & 78800 \\
\hline 50 & 73 & 450 & 0,90 & 0,11 & 0,90 & 1800 & 80600 \\
\hline
\end{tabular}


QUADRO 4.4 - POPULACAO PLANEJADA \& (Concluszo)

\begin{tabular}{|c|c|c|c|c|c|c|c|}
\hline $\begin{array}{l}\text { WOLERO DE } \\
\text { ORDEM }\end{array}$ & $\begin{array}{l}\text { RWIERO DA } \\
\text { OPA }\end{array}$ & $\begin{array}{l}\text { ROAERO DE } \\
\text { DOHICILIOS }\end{array}$ & $\begin{array}{l}\text { TAXA DE } \\
\text { RESPOSTA }\end{array}$ & $\begin{array}{l}\text { DERS. IMTRA. } \\
\text { DE CRIAMCAS }\end{array}$ & $\begin{array}{l}\text { COBERTURA } \\
\text { VACIIIAL }\end{array}$ & $\begin{array}{l}\text { POPULACAO } \\
\text { DA UPA }\end{array}$ & $\begin{array}{l}\text { POPULACAO } \\
\text { ACUHULADA }\end{array}$ \\
\hline $\begin{array}{l}51 \\
52 \\
53\end{array}$ & $\begin{array}{l}75 \\
51 \\
59\end{array}$ & $\begin{array}{l}200 \\
450 \\
200\end{array}$ & $\begin{array}{l}0,90 \\
0,80 \\
0,95\end{array}$ & $\begin{array}{l}0,11 \\
0,12 \\
0,12\end{array}$ & $\begin{array}{l}0,98 \\
0,90 \\
0,98\end{array}$ & $\begin{array}{r}800 \\
1800 \\
800\end{array}$ & $\begin{array}{l}81400 \\
83200 \\
84000\end{array}$ \\
\hline $\begin{array}{l}54 \\
55\end{array}$ & $\begin{array}{r}49 \\
1\end{array}$ & $\begin{array}{l}300 \\
200\end{array}$ & $\begin{array}{l}0,90 \\
0,95\end{array}$ & $\begin{array}{l}0,12 \\
0,16\end{array}$ & $\begin{array}{l}0,90 \\
0,98\end{array}$ & $\begin{array}{l}1200 \\
1200\end{array}$ & $\begin{array}{l}85200 \\
86400\end{array}$ \\
\hline 56 & 60 & 300 & 0,95 & 0,12 & 0,98 & 1200 & 87600 \\
\hline 57 & 9 & 600 & 0,85 & 0,16 & 0,98 & 3600 & 91200 \\
\hline 58 & 72 & 450 & 0,85 & 0,11 & 0,90 & 1800 & 93000 \\
\hline 59 & 21 & 450 & 0,85 & 0,15 & 0,98 & 2475 & 95475 \\
\hline 60 & 55 & 600 & 0,90 & 0,12 & 0,90 & 2400 & 97875 \\
\hline 61 & 76 & 200 & 0,95 & 0,11 & 0,98 & 800 & 98675 \\
\hline 62 & 56 & 200 & 0,90 & 0,12 & 0,98 & 800 & 99475 \\
\hline 63 & B & 450 & 0,95 & 0,16 & 0,98 & 2700 & 102175 \\
\hline 64 & 91 & 600 & 0,85 & 0,10 & 0,90 & 2100 & 104275 \\
\hline 65 & 64 & 450 & 0,90 & 0,12 & 0,98 & 1800 & 106075 \\
\hline 66 & 43 & 450 & 0,85 & 0,13 & 0,98 & 2025 & 108100 \\
\hline 67 & 28 & 300 & 0,85 & 0,14 & 0,98 & 1500 & 109600 \\
\hline 68 & 93 & 200 & 0,95 & 0,14 & 0,98 & 1000 & 110600 \\
\hline 69 & 11 & 600 & 0,90 & 0,16 & 0,98 & 3600 & 114200 \\
\hline 70 & 50 & 300 & 0,95 & 0,12 & 0,90 & 1200 & 115400 \\
\hline 71 & 16 & 200 & 0,95 & 0,15 & 0,98 & 1100 & 116500 \\
\hline 72 & 99 & 600 & 0,80 & 0,10 & 0,98 & 2100 & 118600 \\
\hline 73 & 54 & 600 & 0,85 & 0,12 & 0,90 & 2400 & 121000 \\
\hline 74 & 88 & 300 & 0,95 & 0,10 & 0,90 & 1050 & 122050 \\
\hline 75 & 2 & 300 & 0,80 & 0,16 & 0,98 & 1800 & 123850 \\
\hline 76 & 10 & 600 & 0,90 & 0,16 & 0,98 & 3600 & 127450 \\
\hline 77 & 44 & 300 & 0,95 & 0,13 & 0,98 & 1350 & 128800 \\
\hline 78 & 45 & 300 & 0,95 & 0,13 & 0,98 & 1350 & 130150 \\
\hline 79 & 35 & 300 & 0,85 & 0,13 & 0,90 & 1350 & 131500 \\
\hline 80 & 38 & 450 & 0,80 & 0,13 & 0,90 & 2025 & 133525 \\
\hline 81 & 94 & 300 & 0,80 & 0,10 & 0,98 & 1050 & 134575 \\
\hline 82 & 22 & 450 & 0,85 & 0,15 & 0,98 & 2475 & 137050 \\
\hline 83 & 31 & 450 & 0,95 & 0,14 & 0,98 & 2250 & 139300 \\
\hline 84 & 4 & 300 & 0,90 & 0,16 & 0,98 & 1800 & 141100 \\
\hline 85 & 66 & 600 & 0,85 & 0,12 & 0,98 & 2400 & 143500 \\
\hline 86 & 86 & 200 & 0,95 & 0,16 & 0,98 & 1200 & 144700 \\
\hline 87 & 100 & 600 & 0,85 & 0,10 & 0,98 & 2100 & 146800 \\
\hline 88 & 87 & 300 & 0,90 & 0,10 & 0,90 & 1050 & 147850 \\
\hline 89 & 19 & 300 & 0,90 & 0,15 & 0,98 & 1650 & 149500 \\
\hline 90 & 12 & 600 & 0,90 & 0,16 & 0,98 & 3600 & 153100 \\
\hline 91 & 37 & 300 & 0,95 & 0,13 & 0,90 & 1350 & 154450 \\
\hline 92 & 81 & 300 & 0,95 & 0,11 & 0,98 & 1200 & 155650 \\
\hline 93 & 14 & 200 & 0,80 & 0,15 & 0,98 & 1100 & 156750 \\
\hline 94 & 40 & 600 & 0,90 & 0,13 & 0,90 & 2700 & 159450 \\
\hline 95 & 20 & 300 & 0,95 & 0,15 & 0,98 & 1650 & 161100 \\
\hline 96 & 90 & 450 & 0,90 & 0,10 & 0,90 & 1575 & 162675 \\
\hline 97 & 47 & 600 & 0,95 & 0,13 & 0,98 & 2700 & 165375 \\
\hline 98 & 79 & 300 & 0,85 & 0,11 & 0,98 & 1200 & 166575 \\
\hline 99 & 67 & 200 & 0,85 & 0,11 & 0,90 & 800 & 167375 \\
\hline 100 & 24 & 600 & 0,85 & 0,15 & 0,98 & 3295 & 170670 \\
\hline
\end{tabular}


Figura 4.1 - Distribuição dos conglomerados segundo o número de domicílios, densidade intradomiciliar de criança, cobertura vacinal e taxa de resposta da populaçāo 1.

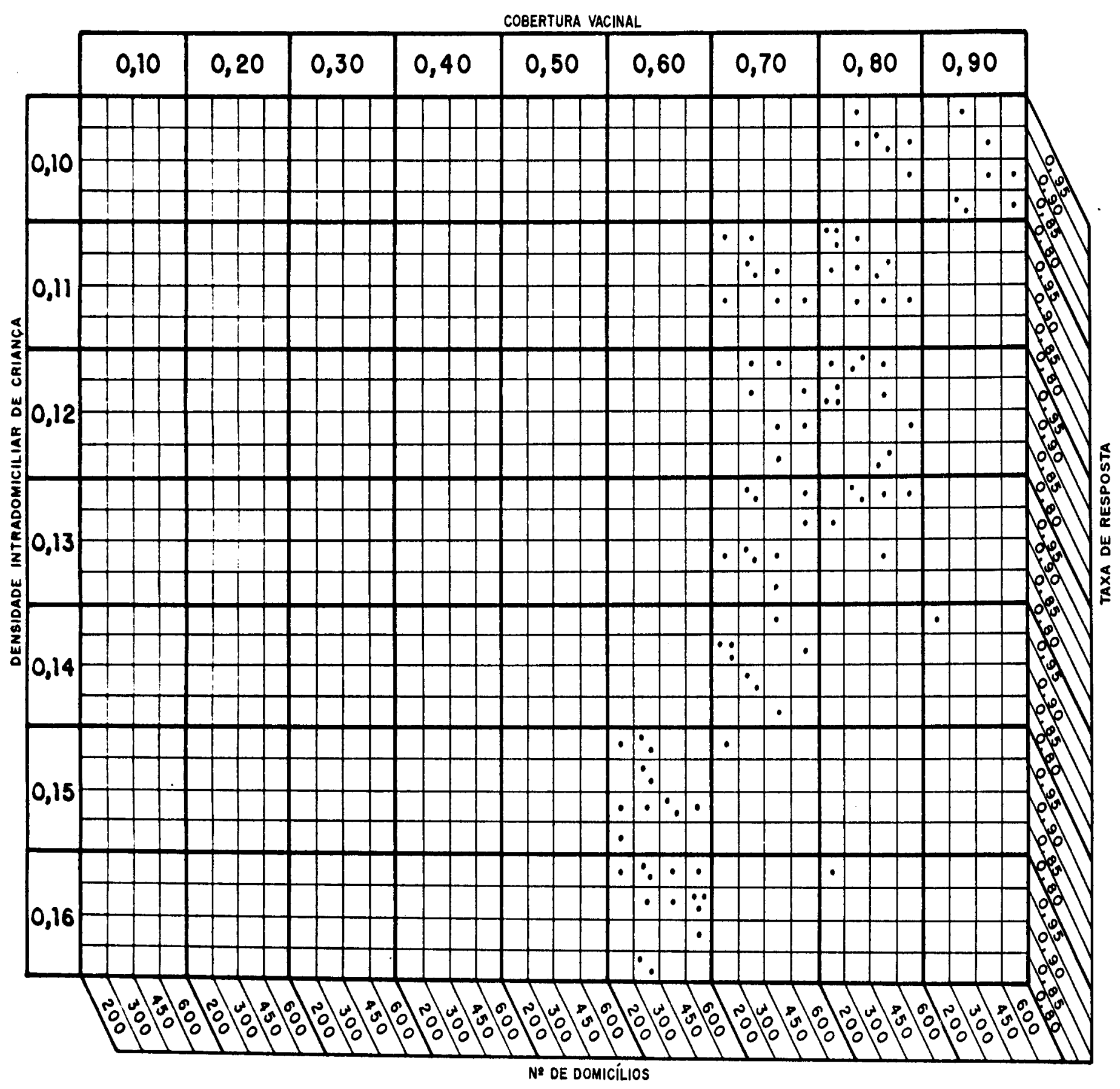


Figuro 4.2 - Distribuição dos conglomerados segundo o número de domicílios, densidade intradomiciliar de criança, cobertura vacinal e taxo de resposto do populaçōo 2.

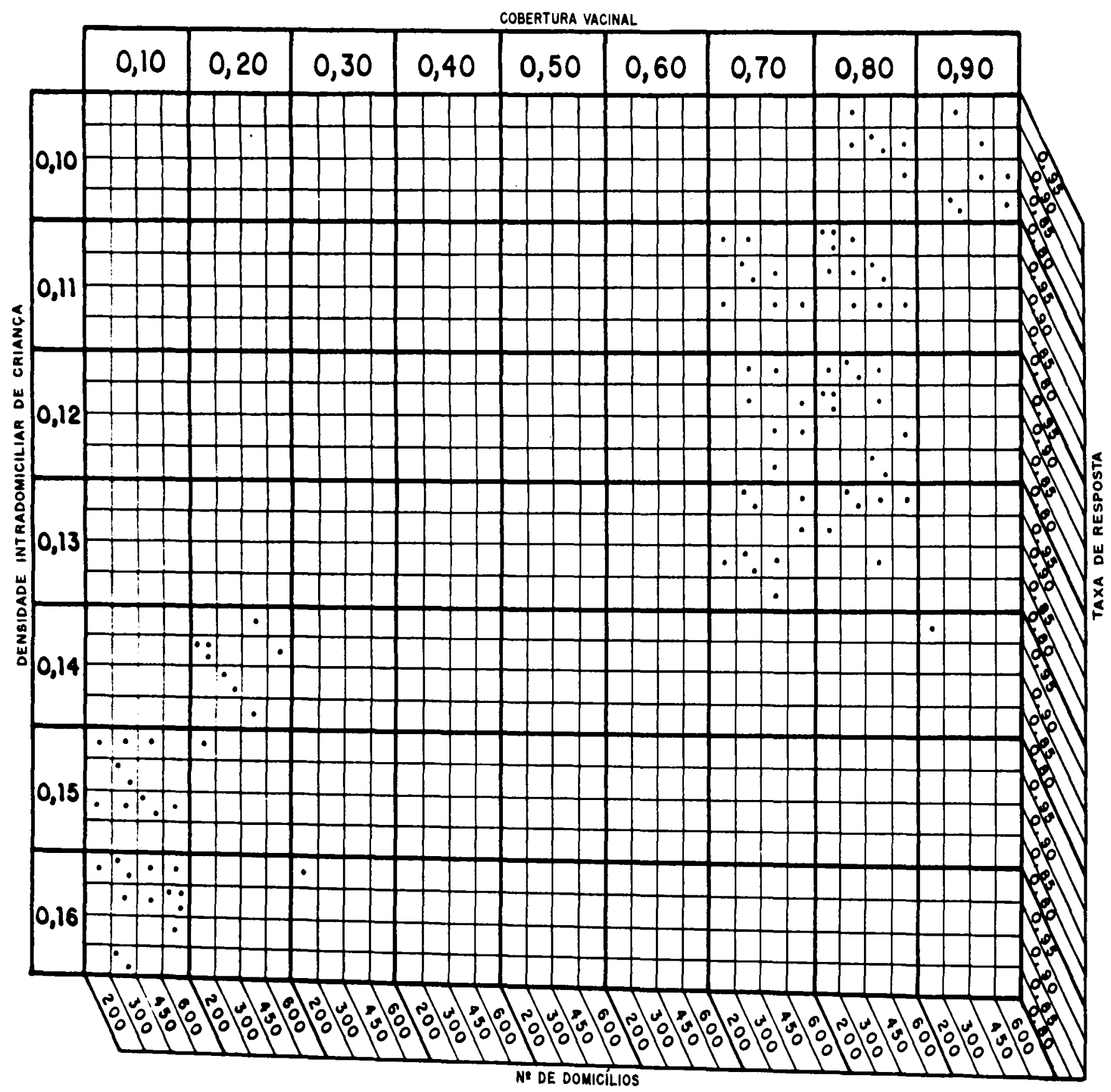


Figuro 4.3 - Distribuição dos conglomerados segundo o número de domicílios, densidade intradomiciliar de criança, cobertura vacinal e taxa de resposta da populaçāo 3.

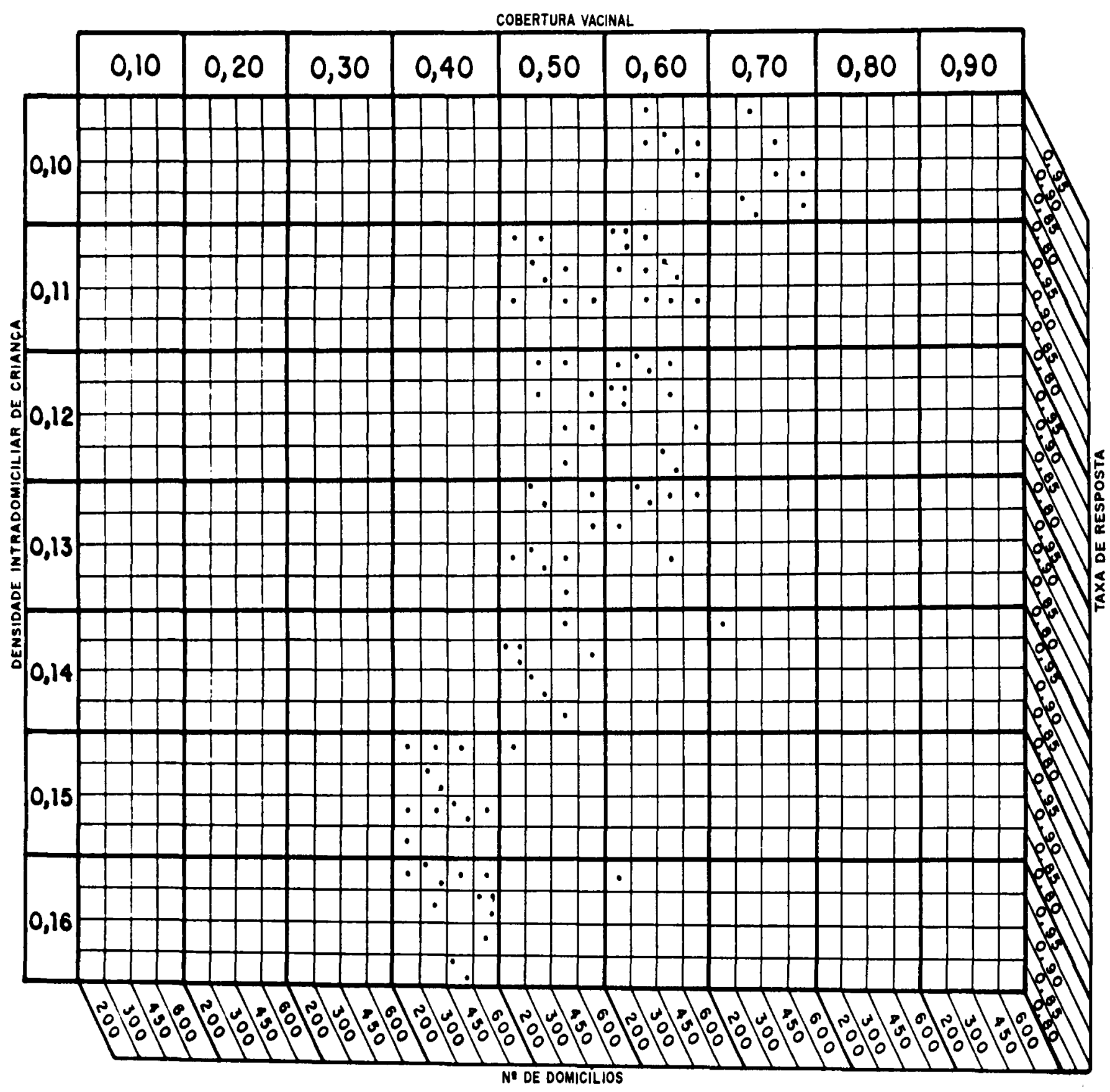


Figuro 4.4 - Distribuiçāo dos conglomerados segundo o número de domicílios, densidade intradomiciliar de criança, cobertura vacinal e taxa de resposta da população 4.

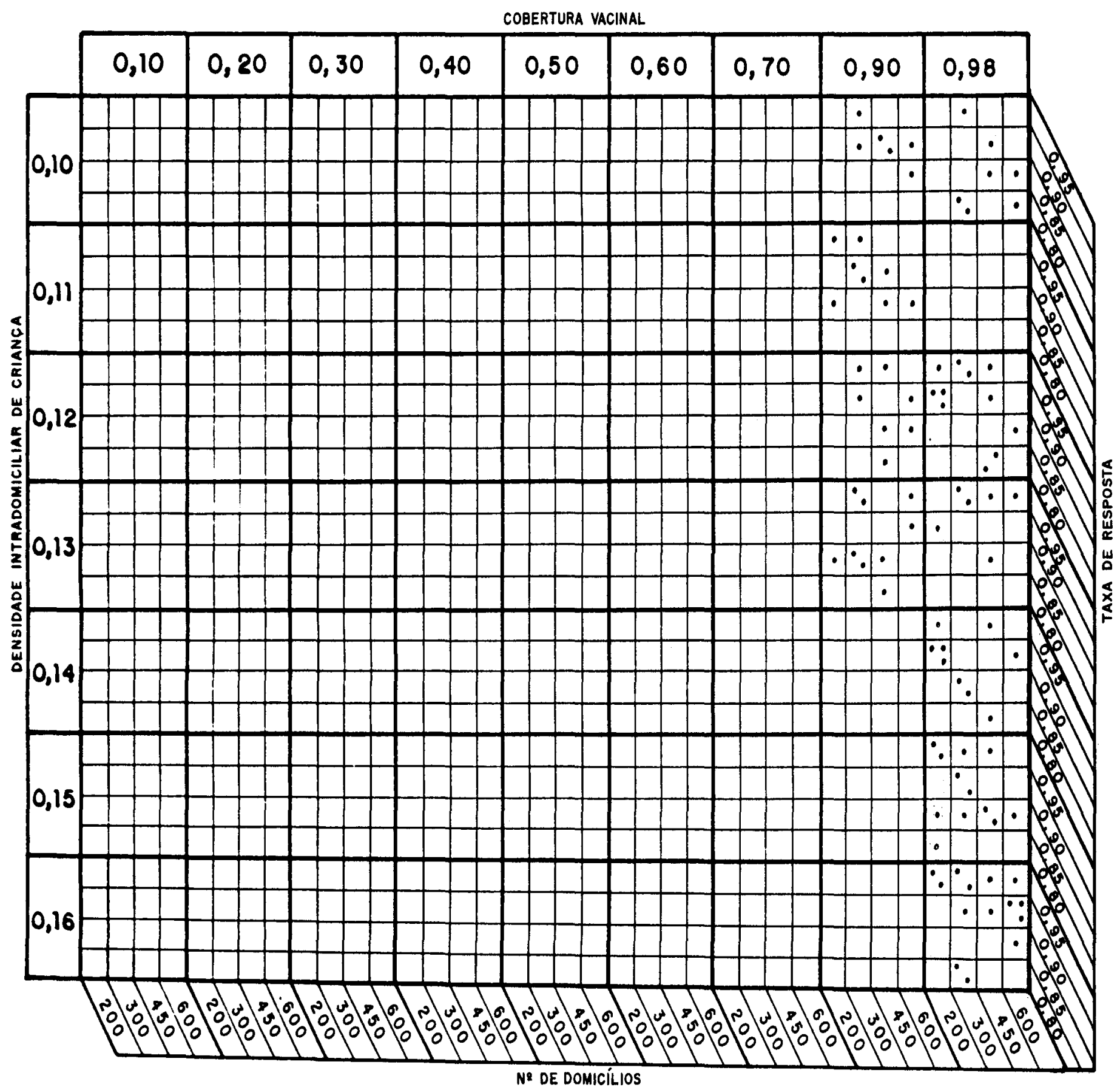




\subsection{Processo da Simulação}

\subsubsection{Os conglomerados e as subamostras}

O conglomerado, com uma combinação especifica de parâmetros, assim como o procedimento de amostragem foram simulados conjuntamente, ou seja, elaboraram-se três programas em BASIC utilizando-se a técnica modular (neste caso foram projetadas duas unidades inter-relacionadas que formaram um programa completo). Os programas utilizaram um gerador de números aleatórios uniformes $[0,1]$ baseado no algoritmo descrito por Naylor ${ }^{14}$ que requer duas sementes que foram sorteadas através de uma Tabela de Números Aleatórios.

Para a construção do conglomerado, isto é, do conjunto de um número determinado de domicílios com uma proporção específica deles possuindo crianças da faixa etária de interesse, foi usado o modelo de distribuição de probabilidades de Poisson, que se ajusta a uma situação de contagem do número de vezes que ocorre um evento em determinado espaço, como pode ser entendido o fenômeno da localização das crianças nos domicílios de um conglomerado.(1) Para a simulação de valores que seguem uma distribuição de Poisson acompanhou-se a seguinte sequência ${ }^{3}$ :

1) A função de probabilidade para a distribuição de Poisson é:

$$
f(x)=\frac{\lambda^{x} e^{-\lambda}}{x !}
$$

2) A função de distribuição de probabilidade é:

$$
F(x)=\sum_{i=0}^{x} \frac{\lambda^{i} e^{-\lambda}}{i !}
$$

\footnotetext{
${ }^{(1}$ Deve-se assumir dois pressupostos para a aplicação do modelo:

$1^{\circ}$ ) o fato de um domicílio ter ou não criança é independente daquilo que ocorre em qualquer outro domicilio $e$

$2^{\circ}$ ) a densidade intradomiciliar de crianças lambda é constante.
} 
3) Valores sucessivos da função de probabilidade $f(x)$ são relacionados por

$$
\begin{aligned}
f(x+1) & =\lambda \frac{f(x)}{(x+1)} \\
f(0) & =e^{-\lambda}
\end{aligned}
$$

para $x=0,1,2, \ldots$

4) Calcula-se a função de distribuição $\mathrm{F}(\mathrm{x})$ para $\mathrm{x}=0,1,2, \ldots, \mathrm{N}$ onde $N$ é o número total de domicílios no conglomerado. Selecionam-se números aleatórios $R$ da distribuição uniforme $[0,1)$. Se $F(x) \leq R<F(x+1)$ então o número aleatório de uma distribuição de Poisson é $x$.

Para a definição da situação vacinal da(s) criança(s) de um domicílio com criança foi utilizado o modelo de distribuição de probabilidades Binomial, dado que se está a frente de uma situação dicotômica - a criança pode ser vacinada ou não vacinada - deve-se, todavia, admitir a suposição de que a probabilidade p de uma criança ser vacinada é constante e que este evento é independente. A sequência descrita a seguir foi utilizada para a geração de um número aleatório de uma distribuição Binomial ${ }^{3}$

1) A função de probabilidade para a distribuição Binomial é:

$$
f(x)=\left(\begin{array}{l}
n \\
x
\end{array}\right) p^{x} \cdot q^{n-x}
$$

2) A função de distribuição de probabilidade é:

$$
F(x)=\sum_{i=0}^{x}\left(\begin{array}{l}
n \\
i
\end{array}\right) p^{i} \cdot q^{n-i}
$$

3) Valores sucessivos da função de probabilidade $f(x)$ são relacionados por:

$$
f(x+1)=\frac{f(x)(n-x) p}{(x+1)(1-p)}
$$


4) Calcula-se a função de distribuição $F(x)$ para $\mathrm{x}=0,1,2, \ldots, \mathrm{N}$ onde $N$ é o número total de crianças no domicilio. Selecionam-se números aleatórios $R$ da distribuição uniforme $[0,1)$. Se $F(x) \leq R<F(x+1)$ então o número aleatório de uma distribuição Binomial é $x$.

Após obter, desta maneira, um conglomerado onde existem domicílios sem criança e com criança e ainda a definição de quais destas crianças são vacinadas, os programas seguem calculando a proporção de crianças vacinadas na população (1) e estabelecem os domicílios não respondentes. Para uma determinada taxa de resposta os programas providenciam uma série de números aleatórios (quantidade de números aleatórios $=$ [tamanho do conglomerado * $(100$ - a\%)] onde a\% é a taxa de resposta) entre 1 e o tamanho do conglomerado, inclusive, que corresponderão aos números dos domicílios no conglomerado os quais serão identificados como não respondentes.

Evidentemente até esta etapa os três programas são iguais, mas para a fase seguinte os algoritmos elaborados são reponsáveis pela seleção de todas as possiveis subamostras de crianças do conglomerado segundo o procedimento de amostragem específico. Assim, para o caso do método de Henderson e Sundaresan todo domicílio do conglomerado tem a oportunidade de ser o "domicílio inicial". A partir deste ponto inicial o conglomerado é "percorrido" com o auxílio de uma variável auxiliar e este percurso termina quando são encontradas sete crianças. Concomitantemente, é contado o número de domicílios percorridos. Com a aplicação desta técnica obtem-se tantas possiveis amostras quanto for o número de domicílios no conglomerado. Observa-se que quando o domicílio inicial estiver localizado no final do conglomerado, o percurso continua no início deste como se fosse circular. A Figura 4.5 representa um conglomerado e a indicação das possíveis amostras pelo método de Henderson e Sundaresan.

No método de Szwarcwald e Valente a sequêencia de passos deve iniciar com uma contagem dos domicílios respondentes com crianças (censo do conglomerado). $\mathrm{O}$ número obtido nesta contagem é dividido por sete e o resultado corresponde ao intervalo a ser aplicado na amostragem sistemática para o sorteio da subamostra de crianças. Para que se posssa obter todas as possíveis subamostras o programa atribui a cada variável denominada "início

\footnotetext{
(Devido à aleatoriedade do processo a proporção de crianças vacinadas pode não ser igual àquela planejada para o conglomerado.
} 


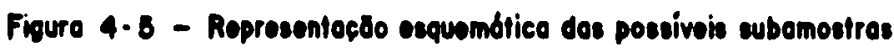

do 7 eriences do UPA I
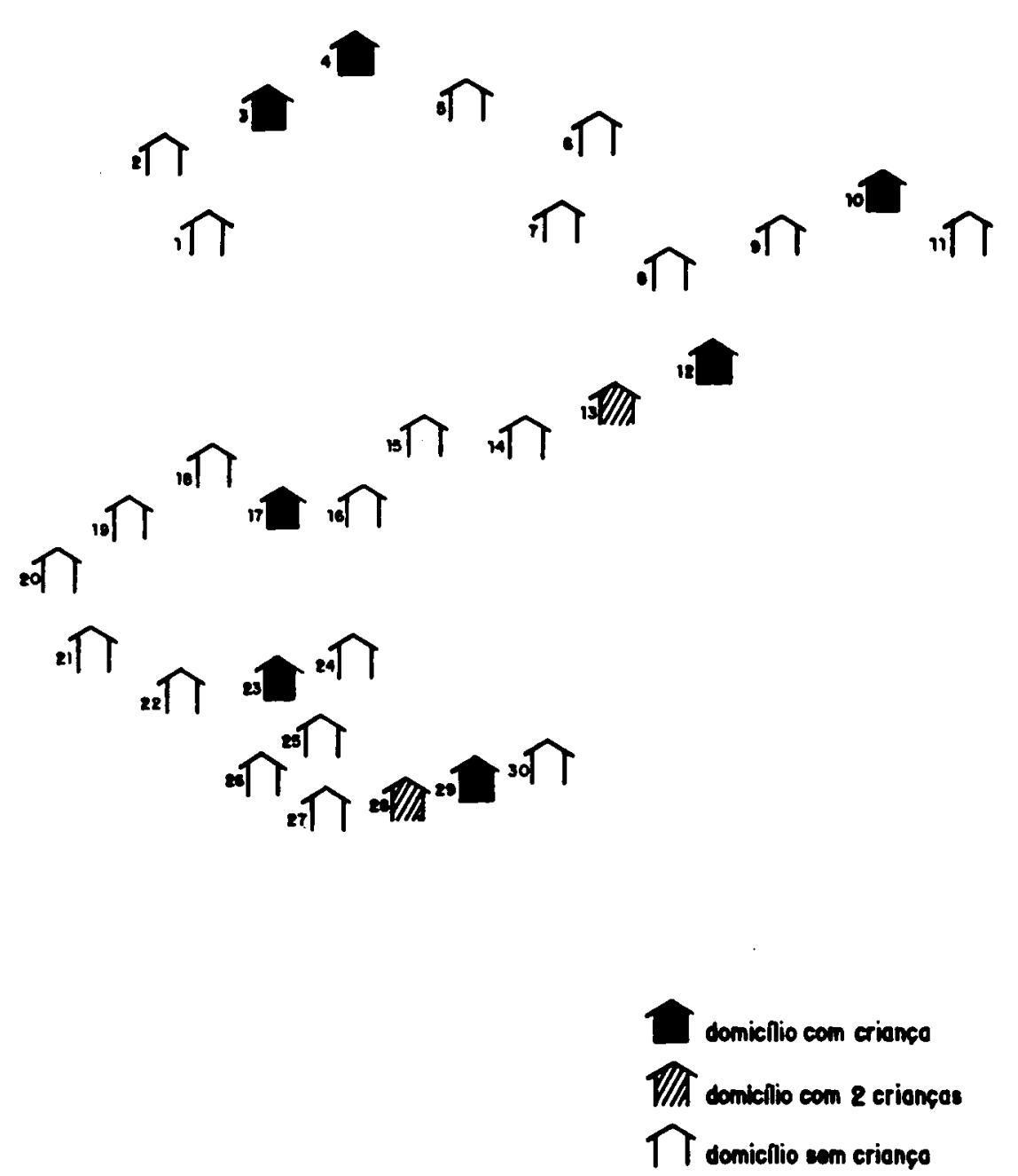

Subamostras:

no de no do domicillio

$1 \quad 1-2-3-4-5-6-7-8-9-10-11-12-13-14-15-16-17$

2 2-3-4-5-6-7-8-9-10-11-12-13-14-15-16-17

$3 \quad 3-4-5-6-7-8-9-10-11-12-13-14-15-16-17$

4 4-5-6-7-8-9-10-11-12-13-14-15-16-17-18-19-20-21-22-23

$\vdots$

$28 \quad 28-29-30-1-2-3-4-5-6-7-8-9-10-11-12$

$29 \quad 29-30-1-2-3-4-5-6-7-8-9-10-11-12-13$

$30 \quad 30-1-2-3-4-5-6-7-8-9-10-11-12-13-14-15-16-17$ 
casual" os valores do intervalo $\left[0,1, I S A_{i}\right]^{11}$, onde $I S A_{i}$ é o intervalo de subamostragem da UPA $i$, com incremento de 0,1 . Desta forma são sorteadas todas as possíveis $\left(I S A_{i} * 10\right)$ subamostras de sete domicílios com crianças dentre o total de domicílios que possuem crianças na UPA $i$.

0 método de Silva difere do anterior no sentido de que não é necessário ser realizado o censo do conglomerado. A partir da fração global de amostragem o programa calcula o intervalo de subamostragem do conglomerado e o restante da seqüência é igual. Desta maneira, são sorteadas todas as possíveis $\left(I S A_{i} * 10\right)$ subamostras de $b$ domicílios com crianças dentre o total de domicílios que possuem crianças na UPA $\mathrm{i}$.

Nos três programas, ao mesmo tempo em que as subamostras são constituidas vão sendo contadas, para cada uma delas, o número total de crianças e quantas destas são vacinadas.

Os programas acima citados são apresentados no Anexo 1.

\subsubsection{As amostras de 30 conglomerados}

Para a seleção de todas as possiveis amostras de 30 UPA's foi escrito um programa em PASCAL que calcula o intervalo de amostragem (total de domicílios da população dividido por 30 ) e atribui valores do intervalo $[1, I A]$, onde IA é o intervalo de amostragem. Considerando que uma amostra de 30 UPA's aparece mais de uma vez, o programa também conta o número de vezes que uma mesma amostra foi selecionada.

0 Anexo 1 apresenta este programa.

\subsection{Análise de dados}

Os dados obtidos pela simulação foram analisados em etapas devido a inviabilidade de realizar todas as possíveis combinações de subamostras de crianças e amostras de UPA's. Consequentemente, a seqüência descrita a seguir foi acompanhada no sentido de obter a melhor aproximação para a Esperança e a Variância de cada distribuição amostral.

\footnotetext{
${ }^{(1}$ se $o$ intervalo de amostragem corresponder a um número inteiro o intervalo será $\left[1, I S A_{i}\right]$
} 
1) Cálculo da estimativa de todas as possiveis subamostras da UPA i

$$
r_{j i}=\frac{y_{j i}}{x_{j i}}
$$

onde

$y_{j i}:$ número de crianças vacinadas na subamostra $j$ da UPA $i$

$x_{j i}$ : número total de crianças na subamostra $j$ da UPA $i$

2) Cálculo da Esperança e Variância da UPA i

$$
\begin{gathered}
E\left(r_{i}\right)=\sum_{j=1}^{b} r_{j i} \cdot \operatorname{prob}_{j i} \\
\operatorname{Var}\left(r_{i}\right)=\sum_{j=1}^{b}\left[r_{j i}-E\left(r_{i}\right)\right]^{2} \cdot \operatorname{prob}_{j i}
\end{gathered}
$$

onde

$b$ : número de todas as possíveis subamostras da UPA i

$\operatorname{prob}_{j i}$ : probabilidade da subamostra $j$ da UPA i ser selecionada

Esta probabilidade prob $_{j i}$ é calculada de maneira diferente segundo o método de amostragem empregado. Assim, tem-se:

- Método de Henderson e Sundaresan

$$
\operatorname{prob}_{j i}=\frac{n d a_{j i}}{n s a_{i}}
$$

onde

$n d a_{j i}$ : número de domicílios sem crianças ou não respondentes existentes entre um domicílio com criança e o domicílio inicial inclusive.

$n s a_{i}$ : número total de subamostras da UPA i

- Método de Szwarcwald e Valente

$$
\operatorname{prob}_{j i}=\frac{1}{n s a_{i}}
$$


- Método de Silva

$$
\operatorname{prob}_{j i}=\frac{1}{n s a_{i}}
$$

3) Cálculo da Esperança e Variância para o valor populacional R

$$
r_{w}=\frac{\sum_{i=1}^{30} E\left(r_{i}\right)}{30}
$$

onde $r_{w}$ é calculado para todas as possiveis amostras de 30 UPA's

$$
\begin{gathered}
E(r)=\sum_{w=1}^{a} r_{w} \cdot p r o b_{w} \\
\operatorname{Var}(r)=\sum_{w=1}^{a}\left[r_{w}-E(r)\right]^{2} \cdot p r o b_{w}
\end{gathered}
$$

onde

$a$ : número de todas as possiveis amostras de 30 UPA's

prob $_{w}$ : probabilidade de seleção da amostra $w$

A probabilidade prob $_{w}$ é calculada considerando-se que as UPA's são selecionadas com probabilidade proporcional ao tamanho (PPT). Assim, tem-se:

onde:

$$
\operatorname{prob}_{w}=\frac{n a_{w}}{n t a}
$$

$n a_{w}$ : número de vezes que a amostra $w$ é selecionada

nta : número total de amostras de 30 UPA's da população

Considerando-se a esperança do estimador-razão pode-se calcular o vício deste estimador para cada procedimento, que é dado pela expressão:

$$
\text { Vicio }(r)=E(r)-R
$$

$\mathrm{E}$, finalmente, se obtem a exatidão para cada procedimento que é dada pelo Erro Quadrático Médio, cuja expressão é:

$$
[E Q M(r)]^{2}=\sigma_{r}^{2}+[E(r)-R]^{2}
$$

Os programas escritos para a análise de dados são apresentados no Anexo 2. 


\section{RESULTADOS E DISCUSSÃO}

Devido à aleatoriedade do processo de simulação, a cobertura vacinal e a densidade intradomiciliar de crianças não ficaram exatamente iguais àquelas planejadas para os conglomerados. Os Quadros 5.1 a 5.4 apresentam os valores realmente obtidos para cada população.

As Tabelas 5.1 a 5.4 apresentam as distribuições amostrais do estimadorrazão e da sua variância relativas aos três métodos de amostragem para as quatro populações respectivamente. Nas últimas linhas destas tabelas se encontram a esperança e a variância do estimador-razão que, como pode ser verificado, não são significativamente diferentes.

$\mathrm{O}$ vício do estimador em cada procedimento de amostragem para as populações simuladas está apresentado no Quadro 5.5. Nota-se neste quadro que a maioria dos valores dos vícios são bem próximos. Chama a atenção, porém, um valor encontrado para a População 3, que é aquela que possui todos os conglomerados com cobertura vacinal em torno de $50 \%$. Nesta população o vício para o estimador-razão no método de Henderson e Sundaresan (PAI) é praticamente o dobro daqueles calculados para os outros métodos. Por outro lado, para a População 4, que tem uma alta cobertura vacinal, o método do PAI é o que produz o menor vício.

Em relação à exatidão dos procedimentos de amostragem, que é medida pelo Erro Quadrático Médio, pode-se dizer que é aproximadamente a mesma para todos os métodos, como pode ser visto no Quadro 5.6.

$\mathrm{O}$ desenho da simulação das populaçōes foi realizado em etapas, ou seja, os conglomerados foram gerados separadamente e para cada um deles foram calculadas a esperança e a variância do estimador da proporção de crianças vacinadas. Este fato proporcionou a possibilidade de se verificar se o vício do estimador- razão se comportava de maneira diferente em função do tipo do conglomerado. Para isto se utilizou o Teste de Kruskal-Wallis.

Inicialmente foi observado se algum dos três métodos produzia um vício maior para os conglomerados isoladamente. $O$ resultado está apresentado no Quadro 5.7. Nota-se, neste quadro, que o valor da estatística usada na prova de Kruskal-Wallis ${ }^{19}$ foi igual a 0,0365 e que forneceu um nivel descritivo de 0,9819. Com base neste resultado conclui-se que não existe evidência de que algum dos métodos esteja provocando um vício maior.

Em seguida, este teste foi aplicado para verificar se os vícios em cada

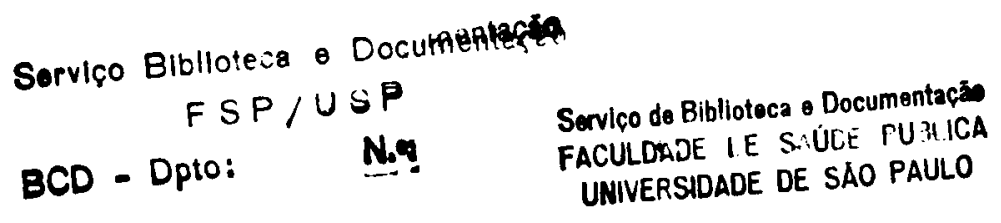


método de amostragem diferiam em função do tamanho do conglomerado, da cobertura vacinal, da densidade intradomiciliar de crianças $\mathrm{e}$ da taxa de resposta. Os Quadros 5.8 a 5.11 apresentam os resultados obtidos. Novamente, pode-se concluir com base nos resultados destes testes que não há evidências de que estes parâmetros relacionados, assim como a taxa de resposta, estejam vinculados com as diferentes magnitudes de vícios.

Para a implementação do método de Szwarcwald e Valente e do método de Silva, o entrevistador deve necessariamente percorrer todo o conglomerado para obter a subamostra de sete crianças. Já no método de Henderson e Sundaresan isto não ocorre. $O$ entrevistador partindo do domicílio inicial percorre somente o suficiente para encontrar as sete crianças. Sendo assim, foi realizada uma compilação do número de domicilios "visitados" para a obtenção das subamostras pelo método do PAI, segundo o tamanho dos conglomerados, para cada população em estudo. O Quadro 5.12 apresenta o número médio de domicílios visitados e também os valores máximos e mínimos. Como pode ser visto, em nenhuma situação o conglomerado inteiro é percorrido, e ainda, os valores mínimos indicam que algumas subamostras podem ser selecionadas a partir de um número muito pequeno de domicílios visitados pelo entrevistador. Este fato, naturalmente, conduz a uma redução significativa nos custos de um levantamento. 
QULDRO 5.1 - POPULACIO SIMULADA 1

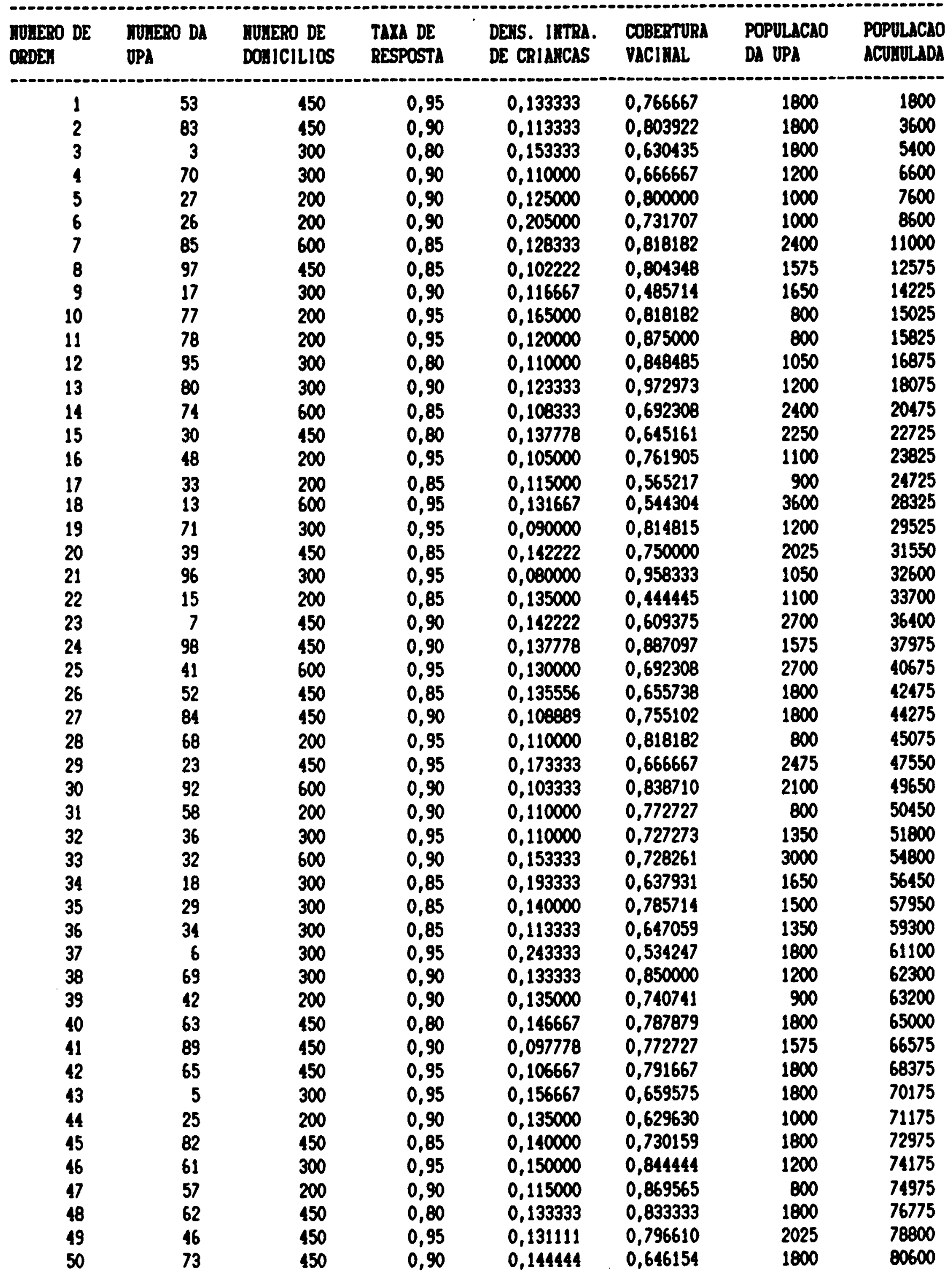


QUADBO 5.1 - POPULACAO SIBULADA I (Conclusso)

\begin{tabular}{|c|c|c|c|c|c|c|c|}
\hline $\begin{array}{l}\text { WOLERO DE } \\
\text { ORDEN }\end{array}$ & $\begin{array}{l}\text { MUAERO DA } \\
\text { UPA }\end{array}$ & $\begin{array}{l}\text { MOLEPO DE } \\
\text { DOAICILIOS }\end{array}$ & $\begin{array}{l}\text { TAXA DE } \\
\text { RESPOSTA }\end{array}$ & $\begin{array}{l}\text { DERS. INTRA. } \\
\text { DE CRIANCAS }\end{array}$ & $\begin{array}{l}\text { COBERTORA } \\
\text { VACIMAL }\end{array}$ & $\begin{array}{l}\text { POPULACAO } \\
\text { DA UPA }\end{array}$ & $\begin{array}{l}\text { POPULACAO } \\
\text { ACURULADA }\end{array}$ \\
\hline 51 & 75 & 200 & 0,90 & 0,125000 & 0,800000 & 800 & 81400 \\
\hline 52 & 51 & 450 & 0,80 & 0,126667 & 0,649123 & 1800 & 83200 \\
\hline 53 & 59 & 200 & 0,95 & 0,120000 & 0,6666667 & 800 & 84000 \\
\hline 54 & 49 & 300 & 0,90 & 0,143333 & 0,651163 & 1200 & 85200 \\
\hline 55 & 1 & 200 & 0,95 & 0,1800000 & 0,555556 & 1200 & 86400 \\
\hline 56 & 60 & 300 & 0,95 & 0,120000 & 0,750000 & 1200 & 87600 \\
\hline 57 & 9 & 600 & 0,85 & 0,1666677 & 0,640000 & 3600 & 91200 \\
\hline 58 & 72 & 450 & 0,85 & 0,111111 & 0,800000 & 1800 & 93000 \\
\hline 59 & 21 & 450 & 0,85 & 0,148889 & 0,671642 & 2475 & 95475 \\
\hline 60 & 55 & 600 & 0,90 & 0,123333 & 0,608108 & 2400 & 97875 \\
\hline 61 & 76 & 200 & 0,95 & 0,100000 & 0,750000 & 800 & 98675 \\
\hline 62 & 56 & 200 & 0,90 & 0,110000 & 0,818182 & 800 & 99475 \\
\hline 63 & 8 & 450 & 0,95 & 0,162222 & 0,630137 & 2700 & 102175 \\
\hline 64 & 91 & 600 & 0,85 & 0,093333 & 0,857143 & 2100 & 104275 \\
\hline 65 & 64 & 450 & 0,90 & 0,135556 & 0,819672 & 1800 & 106075 \\
\hline 66 & 43 & 450 & 0,85 & 0,115556 & 0,692308 & 2025 & 108100 \\
\hline 67 & 28 & 300 & 0,85 & 0,103333 & 0,838710 & 1500 & 109600 \\
\hline 68 & 93 & 200 & 0,95 & 0,100000 & 1,000000 & 1000 & 110600 \\
\hline 69 & 11 & 600 & 0,90 & 0,165000 & 0,545455 & 3600 & 114200 \\
\hline 70 & 50 & 300 & 0,95 & 0,106667 & 0,468750 & 1200 & 115400 \\
\hline 71 & 16 & 200 & 0,95 & 0,195000 & 0,717949 & 1100 & 116500 \\
\hline 72 & 99 & 600 & 0,80 & 0,083333 & 0,860000 & 2100 & 118600 \\
\hline 73 & 54 & 600 & 0,85 & 0,150000 & 0,733333 & 2400 & 121000 \\
\hline 74 & 88 & 300 & 0,95 & 0,103333 & 0,806452 & 1050 & 122050 \\
\hline 75 & 2 & 300 & 0,80 & 0,196667 & 0,644068 & 1800 & 123850 \\
\hline 76 & 10 & 600 & 0,90 & 0,170000 & 0,578431 & 3600 & 127450 \\
\hline 77 & 44 & 300 & 0,95 & 0,1800000 & 0,851852 & 1350 & 128800 \\
\hline 78 & 45 & 300 & 0,95 & 0,153333 & 0,739131 & 1350 & 130150 \\
\hline 79 & 35 & 300 & 0,85 & 0,176667 & 0,716981 & 1350 & 131500 \\
\hline 80 & 38 & 450 & 0,80 & 0,133333 & 0,700000 & 2025 & 133525 \\
\hline 81 & 94 & 300 & 0,80 & 0,093333 & 0,964286 & 1050 & 134575 \\
\hline$B 2$ & 22 & 450 & 0,85 & 0,171111 & 0,584416 & 2475 & 137050 \\
\hline 83 & 31 & 450 & 0,95 & 0,137778 & 0,661290 & 2250 & 139300 \\
\hline 84 & 4 & 300 & 0,90 & 0,156667 & 0,574468 & 1800 & 141100 \\
\hline 85 & 66 & 600 & 0,85 & 0,125000 & 0,840000 & 2400 & 143500 \\
\hline 86 & 86 & 200 & 0,95 & 0,085000 & 0,823530 & 1200 & 144700 \\
\hline 87 & 100 & 600 & 0,85 & 0,090000 & 0,907407 & 2100 & 146800 \\
\hline 88 & 87 & 300 & 0,90 & 0,160000 & 0,729167 & 1050 & 147850 \\
\hline 89 & 19 & 300 & 0,90 & 0,163333 & 0,591837 & 1650 & 149500 \\
\hline 90 & 12 & 600 & 0,90 & 0,146667 & 0,715909 & 3600 & 153100 \\
\hline 91 & 37 & 300 & 0,95 & 0,133333 & 0,725000 & 1350 & 154450 \\
\hline 92 & 81 & 300 & 0,95 & 0,093333 & 0,678571 & 1200 & 155650 \\
\hline 93 & 14 & 200 & 0,80 & 0,160000 & 0,781250 & 1100 & 156750 \\
\hline 94 & 40 & 600 & 0,90 & 0,148333 & 0,662921 & 2700 & 159450 \\
\hline 95 & 20 & 300 & 0,95 & 0,156667 & 0,638298 & 1650 & 161100 \\
\hline 96 & 90 & 450 & 0,90 & 0,0888889 & 0,825000 & 1575 & 162675 \\
\hline 97 & 47 & 600 & 0,95 & 0,126667 & 0,868421 & 2700 & 165375 \\
\hline 98 & 79 & 300 & 0,85 & 0,106667 & 0,781250 & 1200 & 166575 \\
\hline 99 & 67 & 200 & 0,85 & 0,140000 & 0,642857 & 800 & 167375 \\
\hline 100 & 24 & 600 & 0,85 & 0,131667 & 0,582279 & 3295 & 170670 \\
\hline
\end{tabular}


QUADRO 5.2 - POPULACAO SIBULADA 2

\begin{tabular}{|c|c|c|c|c|c|c|c|}
\hline $\begin{array}{l}\text { WOLERO DE } \\
\text { OBDEX }\end{array}$ & $\begin{array}{l}\text { WULERO DA } \\
\text { UPA }\end{array}$ & $\begin{array}{l}\text { WOLERO DE } \\
\text { DOAICILIOS }\end{array}$ & $\begin{array}{l}\text { TIXA DE } \\
\text { RESPOSTA }\end{array}$ & $\begin{array}{l}\text { DEXS. INTRA. } \\
\text { DE CRIANCAS }\end{array}$ & $\begin{array}{l}\text { COBEPTURA } \\
\text { VACIMAL }\end{array}$ & $\begin{array}{l}\text { POPULACAOCO } \\
\text { DA UPA }\end{array}$ & $\begin{array}{l}\text { POPULACAO } \\
\text { ACULULADA }\end{array}$ \\
\hline 1 & 53 & 450 & 0,95 & 0,133333 & 0,766667 & 1800 & 1800 \\
\hline 2 & 83 & 450 & 0,90 & 0,113333 & 0,803922 & 1800 & 3600 \\
\hline 3 & 3 & 300 & 0,80 & 0,193333 & 0,034483 & 1800 & 5100 \\
\hline 4 & 70 & 300 & 0,90 & 0,110000 & 0,666667 & 1200 & 6600 \\
\hline 5 & 27 & 200 & 0,90 & 0,155000 & 0,161290 & 1000 & 7600 \\
\hline 6 & 26 & 200 & 0,90 & 0,155000 & 0,387097 & 1000 & 8600 \\
\hline 7 & 85 & 600 & 0,85 & 0,128333 & 0,818182 & 2400 & 11000 \\
\hline 8 & 97 & 450 & 0,85 & 0,102222 & 0,804348 & 1575 & 12575 \\
\hline 9 & 17 & 300 & 0,90 & 0,150000 & 0,088889 & 1650 & 14225 \\
\hline 10 & 77 & 200 & 0,95 & 0,165000 & 0,818182 & 800 & 15025 \\
\hline 11 & 78 & 200 & 0,95 & 0,120000 & 0,875000 & 800 & 15825 \\
\hline 12 & 95 & 300 & 0.80 & 0,110000 & 0,848485 & 1050 & 16875 \\
\hline 13 & 80 & 300 & 0,90 & 0,123333 & 0,972973 & 1200 & 18075 \\
\hline 14 & 74 & 600 & 0,85 & 0,108333 & 0,692308 & 2400 & 20475 \\
\hline 15 & 30 & 450 & 0,80 & 0,122222 & 0,200000 & 2250 & 22725 \\
\hline 16 & 48 & 200 & 0,95 & 0,205000 & 0,146342 & 1100 & 23825 \\
\hline 17 & 33 & 200 & 0,85 & 0,115000 & 0,565217 & 900 & 24725 \\
\hline 18 & 13 & 600 & 0,95 & 0,146666 & 0,102273 & 3600 & 28325 \\
\hline 19 & 71 & 300 & 0,95 & 0,090000 & 0,814815 & 1200 & 29525 \\
\hline 20 & 39 & 450 & 0,85 & 0,142222 & 0,750000 & 2025 & 31550 \\
\hline 21 & 96 & 300 & 0,95 & 0,080000 & 0,958333 & 1050 & 32600 \\
\hline 22 & 15 & 200 & 0,85 & 0,130000 & 0,115385 & 1100 & 33700 \\
\hline 23 & 7 & 450 & 0,90 & 0,117777 & 0,094310 & 2700 & 36400 \\
\hline 24 & 98 & 450 & 0,90 & 0,137777 & 0,887097 & 1575 & 37975 \\
\hline 25 & 41 & 600 & 0,95 & 0,130000 & 0,692308 & 2700 & 40675 \\
\hline 26 & 52 & 450 & 0,85 & 0,135555 & 0,655738 & 1800 & 42475 \\
\hline 27 & 84 & 450 & 0,90 & 0,108888 & 0,755102 & 1800 & 44275 \\
\hline 28 & 68 & 200 & 0,95 & 0,110000 & 0,818182 & 800 & 45075 \\
\hline 29 & 23 & 450 & 0,95 & 0,153333 & 0,057971 & 2475 & 47550 \\
\hline 30 & 92 & 600 & 0,90 & 0,103333 & 0,838710 & 2100 & 49650 \\
\hline 31 & 58 & 200 & 0,90 & 0,110000 & 0,772727 & 800 & 50450 \\
\hline 32 & 36 & 300 & 0,95 & 0,110000 & 0,727273 & 1350 & 51800 \\
\hline 33 & 32 & 600 & 0,90 & 0,130000 & 0,230769 & 3000 & 54800 \\
\hline 34 & 18 & 300 & 0,85 & 0,176666 & 0,094340 & 1650 & 56450 \\
\hline 35 & 29 & 300 & 0,85 & 0,166666 & 0,120000 & 1500 & 57950 \\
\hline 36 & 34 & 300 & 0,85 & 0,113333 & 0,647059 & 1350 & 59300 \\
\hline 37 & 6 & 300 & 0,95 & 0,150000 & 0,133333 & 1800 & 61100 \\
\hline 38 & 69 & 300 & 0,90 & 0,133333 & 0,850000 & 1200 & 62300 \\
\hline 39 & 42 & 200 & 0,90 & 0,135000 & 0,740741 & 900 & 63200 \\
\hline 40 & 63 & 450 & 0,80 & 0,146666 & 0,787879 & 1800 & 65000 \\
\hline 11 & 89 & 450 & 0,90 & 0,097777 & 0,772727 & 1575 & 66575 \\
\hline 42 & 65 & 450 & 0,95 & 0,106666 & 0,791667 & 1800 & 68375 \\
\hline 43 & 5 & 300 & 0,95 & 0,186666 & 0,142857 & 1800 & 70175 \\
\hline 44 & 25 & 200 & 0,90 & 0,150000 & 0,266667 & 1000 & 71175 \\
\hline 45 & 82 & 450 & 0,85 & 0,140000 & 0,730159 & 1800 & 72975 \\
\hline 46 & 61 & 300 & 0,95 & 0,150000 & 0,844444 & 1200 & 74175 \\
\hline 47 & 57 & 200 & 0,90 & 0,115000 & 0,869565 & 800 & 74975 \\
\hline 48 & 62 & 450 & 0,80 & 0,133333 & 0,833333 & 1800 & 76775 \\
\hline 49 & 46 & 450 & 0,95 & 0,131111 & 0,796610 & 2025 & 78800 \\
\hline 50 & 73 & 450 & 0,90 & 0,144444 & 0,646154 & 1800 & 80600 \\
\hline
\end{tabular}


QUADRO 5.2 - POPULACAO SIGULADA 2 (Conclugao)

\begin{tabular}{|c|c|c|c|c|c|c|c|}
\hline $\begin{array}{l}\text { ROHERO DE } \\
\text { ORDE\# }\end{array}$ & $\begin{array}{l}\text { RORERO DA } \\
\text { UPA }\end{array}$ & $\begin{array}{l}\text { RUIERO DE } \\
\text { DOAICILIOS }\end{array}$ & $\begin{array}{l}\text { TAXA DE } \\
\text { RESPOSTA }\end{array}$ & $\begin{array}{l}\text { DERS. IITRA, } \\
\text { DE CRIAMCAS }\end{array}$ & $\begin{array}{l}\text { COBERTURA } \\
\text { VACIMAL }\end{array}$ & $\begin{array}{l}\text { POPULACAO } \\
\text { DA UPA }\end{array}$ & $\begin{array}{l}\text { POPOLACAO } \\
\text { ACUHULADA }\end{array}$ \\
\hline 51 & 75 & 200 & 0,90 & 0,125000 & 0,800000 & 800 & 81400 \\
\hline 52 & $\begin{array}{l}51 \\
59\end{array}$ & 450 & $\begin{array}{l}0,80 \\
0.95\end{array}$ & $\begin{array}{l}0,126666 \\
0,120000\end{array}$ & 0,649123 & $\begin{array}{r}1800 \\
800\end{array}$ & $\begin{array}{l}83200 \\
84000\end{array}$ \\
\hline 53 & 59 & 200 & 0,95 & 0,120000 & & 800 & 84000 \\
\hline 54 & 49 & 300 & 0,90 & 0,143333 & 0,651163 & 1200 & 85200 \\
\hline 35 & 1 & 200 & 0,95 & 0,110000 & 0,045455 & 1200 & 86400 \\
\hline 56 & 60 & 300 & 0,95 & 0,120000 & 0,750000 & 1200 & 87600 \\
\hline 57 & 9 & 600 & 0,85 & 0,171666 & 0,116505 & 3600 & 91200 \\
\hline 58 & 72 & 450 & 0,85 & 0,111111 & 0,800000 & 1800 & 93000 \\
\hline $\begin{array}{l}59 \\
60 \\
61\end{array}$ & $\begin{array}{l}21 \\
55 \\
76\end{array}$ & $\begin{array}{l}150 \\
600 \\
200\end{array}$ & $\begin{array}{l}0,85 \\
0,90 \\
0,95\end{array}$ & $\begin{array}{l}0,151111 \\
0,123333 \\
0,100000\end{array}$ & $\begin{array}{l}0,132353 \\
0,608108 \\
0,750000\end{array}$ & $\begin{array}{r}2475 \\
2400 \\
800\end{array}$ & $\begin{array}{l}95475 \\
97875 \\
98675\end{array}$ \\
\hline 62 & 56 & 200 & 0,90 & 0,110000 & 0,818182 & 800 & 99475 \\
\hline 63 & B & 450 & 0,95 & 0,177777 & 0,100000 & 2700 & 102175 \\
\hline 64 & 91 & 600 & 0,85 & 0,093333 & 0,857143 & 2100 & 104275 \\
\hline 65 & 64 & 450 & 0,90 & 0,135555 & 0,819672 & 1800 & 106075 \\
\hline 66 & 43 & 450 & 0,85 & 0,115555 & 0,692308 & 2025 & 108100 \\
\hline 67 & 28 & 300 & 0,85 & 0,163333 & 0,265306 & 1500 & 109600 \\
\hline 68 & 93 & 200 & 0,95 & 0,100000 & 1,000000 & 1000 & 110600 \\
\hline 69 & 11 & 600 & 0,90 & 0,158333 & 0,105263 & 3600 & 114200 \\
\hline 70 & 50 & 300 & 0,95 & 0,106666 & 0,468750 & 1200 & 115400 \\
\hline 71 & 16 & 200 & 0,95 & 0,165000 & 0,060606 & 1100 & 116500 \\
\hline 72 & 99 & 600 & 0,80 & 0,083333 & 0,860000 & 2100 & 118600 \\
\hline 73 & 54 & 600 & 0,85 & 0,150000 & 0,733333 & 2400 & 121000 \\
\hline 74 & 88 & 300 & 0,95 & 0,103333 & 0,806452 & 1050 & 122050 \\
\hline 75 & 2 & 300 & 0,80 & 0,136666 & 0,121951 & 1800 & 123850 \\
\hline 76 & 10 & 600 & 0,90 & 0,178333 & 0,140187 & 3600 & 127450 \\
\hline 77 & 44 & 300 & 0,95 & 0,180000 & 0,851852 & 1350 & 128800 \\
\hline 78 & 45 & 300 & 0,95 & 0,153333 & 0,739131 & 1350 & 130150 \\
\hline 79 & 35 & 300 & 0,85 & 0,176666 & 0,716981 & 1350 & 131500 \\
\hline 80 & 38 & 450 & 0,80 & 0,133333 & 0,700000 & 2025 & 133525 \\
\hline 81 & 94 & 300 & 0,80 & 0,093333 & 0,964286 & 1050 & 134575 \\
\hline 82 & 22 & 450 & 0,85 & 0,140000 & 0,111111 & 2475 & 137050 \\
\hline 83 & 31 & 450 & 0,95 & 0,162222 & 0,164384 & 2250 & 139300 \\
\hline 84 & 4 & 300 & 0,90 & 0,173333 & 0,057692 & 1800 & 141100 \\
\hline 85 & 66 & 600 & 0,85 & 0,125000 & 0,840000 & 2400 & 143500 \\
\hline 86 & 86 & 200 & 0,95 & 0,195000 & 0,282051 & 1200 & 144700 \\
\hline 87 & 100 & 600 & 0,85 & 0,090000 & 0,907407 & 2100 & 146800 \\
\hline 88 & 87 & 300 & 0,90 & 0,160000 & 0,729167 & 1050 & 147850 \\
\hline 89 & 19 & 300 & 0,90 & 0,140000 & 0,142857 & 1650 & 149500 \\
\hline 90 & 12 & 600 & 0,90 & 0,198333 & 0,134454 & 3600 & 153100 \\
\hline 91 & 37 & 300 & 0,95 & 0,133333 & 0,725000 & 1350 & 154450 \\
\hline 92 & 81 & 300 & 0,95 & 0,093333 & 0,678571 & 1200 & 155650 \\
\hline 93 & 14 & 200 & 0,80 & 0,135000 & 0,037037 & 1100 & 156750 \\
\hline 94 & 40 & 600 & 0,90 & 0,148333 & 0,662921 & 2700 & 159450 \\
\hline 95 & 20 & 300 & 0,95 & 0,160000 & 0,187500 & 1650 & 161100 \\
\hline 96 & 90 & 450 & 0,90 & 0,088888 & 0,825000 & 1575 & 162675 \\
\hline 97 & 47 & 600 & 0,95 & 0,126666 & 0,868421 & 2700 & 165375 \\
\hline 98 & 79 & 300 & 0.85 & 0.106666 & 0,781250 & 1200 & 166575 \\
\hline 99 & 67 & 200 & 0,85 & 0,140000 & 0,642857 & 800 & 167375 \\
\hline 100 & 24 & 600 & 0,85 & 0,150000 & 0,077778 & 3295 & 170670 \\
\hline
\end{tabular}


QUADRO 3.3 - POPULACAO SIIULADA 3

\begin{tabular}{|c|c|c|c|c|c|c|c|}
\hline $\begin{array}{l}\text { ROLERSO DE } \\
\text { ORDEX }\end{array}$ & $\begin{array}{l}\text { AULEPD DA } \\
\text { OPA }\end{array}$ & $\begin{array}{l}\text { ROHERO DE } \\
\text { DOHICILIOS }\end{array}$ & $\begin{array}{l}\text { TAXA DE } \\
\text { RESPOSTA }\end{array}$ & $\begin{array}{l}\text { DERS. INTRA. } \\
\text { DE CRIAMCAS }\end{array}$ & $\begin{array}{l}\text { COBERTURA } \\
\text { VACIMAL }\end{array}$ & $\begin{array}{l}\text { POPULACAO } \\
\text { DA UPA }\end{array}$ & $\begin{array}{l}\text { POPULACAO } \\
\text { ACURULADA }\end{array}$ \\
\hline 1 & 53 & 450 & 0.95 & 0.126667 & 0.543860 & 1800 & 1800 \\
\hline 2 & 83 & 450 & 0.90 & 0.113333 & 0.529412 & 1800 & 3600 \\
\hline 3 & 3 & 300 & 0.80 & 0.153333 & 0.413044 & 1800 & 5400 \\
\hline 4 & 70 & 300 & 0.90 & 0.126667 & 0.473684 & 1200 & 6600 \\
\hline 5 & 27 & 200 & 0.90 & 0.145000 & 0.517241 & 1000 & 7600 \\
\hline 6 & 26 & 200 & 0.90 & 0.165000 & 0.363636 & 1000 & 8600 \\
\hline 7 & 85 & 600 & 0.85 & 0.106667 & 0.562500 & 2400 & 11000 \\
\hline 8 & 97 & 450 & 0.85 & 0.075556 & 0.705882 & 1575 & 12575 \\
\hline 9 & 17 & 300 & 0.90 & 0.120000 & 0.333333 & 1650 & 14225 \\
\hline 10 & 77 & 200 & 0.95 & 0.120000 & 0.708333 & 800 & 25025 \\
\hline 11 & 78 & 200 & 0.95 & 0.120000 & 0.625000 & 800 & 15825 \\
\hline 12 & 95 & 300 & 0.80 & 0.106667 & 0.625000 & 1050 & 16875 \\
\hline 13 & 80 & 300 & 0.90 & 0.136667 & 0.585366 & 1200 & 18075 \\
\hline 14 & 74 & 600 & 0.85 & 0.140000 & 0.452381 & 2400 & 20475 \\
\hline 15 & 30 & 450 & 0.80 & 0.144444 & 0.569231 & 2250 & 22725 \\
\hline 16 & 48 & 200 & 0.95 & 0.155000 & 0.354839 & 1100 & 23825 \\
\hline 17 & 33 & 200 & 0.85 & 0.095000 & 0.421053 & 900 & 24725 \\
\hline 18 & 13 & 600 & 0.95 & 0.151667 & 0.384615 & 3600 & 28325 \\
\hline 19 & 71 & 300 & 0.95 & 0.090000 & 0.481482 & 1200 & 29525 \\
\hline 20 & 39 & 450 & 0.85 & 0.126667 & 0.526316 & 2025 & 31550 \\
\hline 21 & 96 & 300 & 0.95 & 0.100000 & 0.633333 & 1050 & 32600 \\
\hline 22 & 15 & 200 & 0.85 & 0.130000 & 0.538462 & 1100 & 33700 \\
\hline 23 & 7 & 450 & 0.90 & 0.184444 & 0.373494 & 2700 & 36400 \\
\hline 24 & 98 & 450 & 0.90 & 0.104444 & 0.744681 & 1575 & 37975 \\
\hline 25 & 41 & 600 & 0.95 & 0.133333 & 0.600000 & 2700 & 40675 \\
\hline 26 & 52 & 450 & 0.85 & 0.126667 & 0.543860 & 1800 & 42475 \\
\hline 27 & 84 & 450 & 0.90 & 0.128889 & 0.672414 & 1800 & 44275 \\
\hline 28 & 68 & 200 & 0.95 & 0.110000 & 0.454546 & 800 & 45075 \\
\hline 29 & 23 & 450 & 0.95 & 0.184444 & 0.373494 & 2475 & 47550 \\
\hline 30 & 92 & 600 & 0.90 & 0.093333 & 0.553571 & 2100 & 49650 \\
\hline 31 & 58 & 200 & 0.90 & 0.120000 & 0.750000 & 800 & 50450 \\
\hline 32 & 36 & 300 & 0.95 & 0.186667 & 0.500000 & 1350 & 51800 \\
\hline 33 & 32 & 600 & 0.90 & 0.150000 & 0.477778 & 3000 & 54800 \\
\hline 34 & 18 & 300 & 0.85 & 0.126667 & 0.368421 & 1650 & 56450 \\
\hline 35 & 29 & 300 & 0.85 & 0.136667 & 0.414634 & 1500 & 57950 \\
\hline 36 & 34 & 300 & 0.85 & 0.130000 & 0.512821 & 1350 & 59300 \\
\hline 37 & 6 & 300 & 0.95 & 0.136667 & 0.268293 & 1800 & 61100 \\
\hline 38 & 69 & 300 & 0.90 & 0.156667 & 0.340426 & 1200 & 62300 \\
\hline 39 & 42 & 200 & 0.90 & 0.135000 & 0.518519 & 900 & 63200 \\
\hline 40 & 63 & 450 & 0.80 & 0.120000 & 0.592593 & 1800 & 65000 \\
\hline 11 & 89 & 450 & 0.90 & 0.082222 & 0.675676 & 1575 & 66575 \\
\hline 42 & 65 & 450 & 0.95 & 0.126667 & 0.614035 & 1800 & 68375 \\
\hline 43 & 5 & 300 & 0.95 & 0.153333 & 0.543478 & 1800 & 70175 \\
\hline 44 & 25 & 200 & 0.90 & 0.115000 & 0.478261 & 1000 & 71175 \\
\hline 45 & 82 & 450 & 0.85 & 0.093333 & 0.666667 & 1800 & 72975 \\
\hline 46 & 61 & 300 & 0.95 & 0.143333 & 0.720930 & 1200 & 74175 \\
\hline 17 & 57 & 200 & 0.90 & 0.085000 & 0.529412 & 800 & 74975 \\
\hline 48 & 62 & 450 & 0.80 & 0.120000 & 0.611111 & 1800 & 76775 \\
\hline 49 & 46 & 450 & 0.95 & 0.124444 & 0.750000 & 2025 & 78800 \\
\hline 50 & 73 & 450 & 0.90 & 0.140000 & 0.492064 & 1800 & 80600 \\
\hline
\end{tabular}


QUADRO 5.3 - POPULACAO SIMULADA 3 (Concluseo)

\begin{tabular}{|c|c|c|c|c|c|c|c|}
\hline $\begin{array}{l}\text { DUIERO DE } \\
\text { ORDEN }\end{array}$ & $\begin{array}{l}\text { ROIERO DA } \\
\text { UPA }\end{array}$ & $\begin{array}{l}\text { DOHERO DE } \\
\text { DOHICILIOS }\end{array}$ & $\begin{array}{l}\text { TAXA DE } \\
\text { RESPOSTA }\end{array}$ & $\begin{array}{l}\text { DERS. INTRA. } \\
\text { DE CRIANCAS }\end{array}$ & $\begin{array}{l}\text { COBERTURA } \\
\text { VACIMAL }\end{array}$ & $\begin{array}{l}\text { POPOLACAO } \\
\text { DA OPA }\end{array}$ & $\begin{array}{l}\text { POPOLACAO } \\
\text { ACUNOLADA }\end{array}$ \\
\hline 51 & 75 & 200 & 0.90 & 0.130000 & 0.653846 & 800 & 81400 \\
\hline 52 & 51 & 450 & 0.80 & 0.128889 & 0.551724 & 1800 & 83200 \\
\hline 53 & 59 & 200 & 0.95 & 0.120000 & 0.500000 & 800 & 84000 \\
\hline 54 & 49 & 300 & 0.90 & 0.120000 & 0.500000 & 1200 & 85200 \\
\hline $5 b$ & 1 & 200 & 0.95 & 0.130000 & 0.346154 & 1200 & 86400 \\
\hline 56 & 60 & 300 & 0.95 & 0.140000 & 0.642857 & 1200 & 87600 \\
\hline 57 & 9 & 600 & 0.85 & 0.163333 & 0.479592 & 3600 & 91200 \\
\hline 58 & 72 & 450 & 0.85 & 0.137778 & 0.548387 & 1800 & 93000 \\
\hline 59 & 21 & 450 & 0.85 & 0.153333 & 0.463768 & 2475 & 95475 \\
\hline 60 & 55 & 600 & 0.90 & 0.133333 & 0.462500 & 2400 & 97875 \\
\hline 61 & 76 & 200 & 0.95 & 0.130000 & 0.538462 & 800 & 98675 \\
\hline 62 & 56 & 200 & 0.90 & 0.190000 & 0.657895 & 800 & 99475 \\
\hline 63 & 8 & 450 & 0.95 & 0.173333 & 0.397436 & 2700 & 102175 \\
\hline 64 & 91 & 600 & 0.85 & 0.088333 & 0.566038 & 2100 & 104275 \\
\hline 65 & 61 & 450 & 0.90 & 0.104444 & 0.531915 & 1800 & 106075 \\
\hline 66 & 43 & 450 & 0.85 & 0.133333 & 0.566667 & 2025 & 108100 \\
\hline 67 & 28 & 300 & 0.85 & 0.170000 & 0.509804 & 1500 & 109600 \\
\hline 68 & 93 & 200 & 0.95 & 0.145000 & 0.689655 & 1000 & 110600 \\
\hline 69 & 11 & 600 & 0.90 & 0.155000 & 0.376344 & 3600 & 114200 \\
\hline 70 & 50 & 300 & 0.95 & 0.133333 & 0.575000 & 1200 & 115400 \\
\hline 71 & 16 & 200 & 0.95 & 0.120000 & 0.500000 & 1100 & 116500 \\
\hline 72 & 99 & 600 & 0.80 & 0.093333 & 0.714286 & 2100 & 118600 \\
\hline 73 & 54 & 600 & 0.85 & 0.130000 & 0.397436 & 2400 & 121000 \\
\hline 74 & 88 & 300 & 0.95 & 0.086667 & 0.692308 & 1050 & 122050 \\
\hline 75 & 2 & 300 & 0.80 & 0.160000 & 0.520833 & 1800 & 123850 \\
\hline 76 & 10 & 600 & 0.90 & 0.168333 & 0.356436 & 3600 & 127450 \\
\hline 77 & 44 & 300 & 0.95 & 0.156667 & 0.659575 & 1350 & 128800 \\
\hline 78 & 45 & 300 & 0.95 & 0.156667 & 0.617021 & 1350 & 130150 \\
\hline 79 & 35 & 300 & 0.85 & 0.140000 & 0.476191 & 1350 & 131500 \\
\hline 80 & 38 & 450 & 0.80 & 0.162222 & 0.465754 & 2025 & 133525 \\
\hline 81 & 94 & 300 & 0.60 & 0.1166667 & 0.657143 & 1050 & 134575 \\
\hline 82 & 22 & 450 & 0.85 & 0.160000 & 0.375000 & 2475 & 137050 \\
\hline 83 & 31 & 450 & 0.95 & 0.153333 & 0.623188 & 2250 & 139300 \\
\hline 84 & 1 & 300 & 0.90 & 0.166667 & 0.440000 & 1800 & 141100 \\
\hline 85 & 66 & 600 & 0.85 & 0.110000 & 0.621212 & 2400 & 143500 \\
\hline 86 & 86 & 200 & 0.95 & 0.140000 & 0.678571 & 1200 & 144700 \\
\hline 87 & 100 & 600 & 0.85 & 0.081667 & 0.755102 & 2100 & 146800 \\
\hline 88 & 87 & 300 & 0.90 & 0.106667 & 0.468750 & 1050 & 147850 \\
\hline 89 & 19 & 300 & 0.90 & 0.166667 & 0.420000 & 1650 & 149500 \\
\hline 90 & 12 & 600 & 0.90 & 0.178333 & 0.336449 & 3600 & 153100 \\
\hline 91 & 37 & 300 & 0.95 & 0.113333 & 0.617647 & 1350 & 154450 \\
\hline 92 & 81 & 300 & 0.95 & 0.090000 & 0.666667 & 1200 & 155650 \\
\hline 93 & 14 & 200 & 0.80 & 0.135000 & 0.259259 & 1100 & 156750 \\
\hline 94 & 40 & 600 & 0.90 & 0.135000 & 0.530864 & 2700 & 159450 \\
\hline 95 & 20 & 300 & 0.95 & 0.173333 & 0.307692 & 1650 & 161100 \\
\hline 96 & 90 & 450 & 0.90 & 0.131111 & 0.610170 & 1575 & 162675 \\
\hline 97 & 47 & 600 & 0.95 & 0.141667 & 0.6588824 & 2700 & 165375 \\
\hline 98 & 79 & 300 & 0.85 & 0.143333 & 0.581395 & 1200 & 166575 \\
\hline 99 & 67 & 200 & 0.85 & 0.155000 & 0.516129 & 800 & 167375 \\
\hline 100 & 24 & 600 & 0.85 & 0.138333 & 0.301205 & 3295 & 170670 \\
\hline
\end{tabular}


QUADRO 5.4 - POPULACAO SIRULADA 4

\begin{tabular}{|c|c|c|c|c|c|c|c|}
\hline $\begin{array}{l}\text { MOLERO DE } \\
\text { ORDEX }\end{array}$ & $\begin{array}{l}\text { ROAERO DA } \\
\text { OPA }\end{array}$ & $\begin{array}{l}\text { ROLERO DE } \\
\text { DOHICILIOS }\end{array}$ & $\begin{array}{l}\text { TAXA DE } \\
\text { RESPOSTA }\end{array}$ & $\begin{array}{l}\text { DENS. IMTRA. } \\
\text { DE CRIAMCAS }\end{array}$ & $\begin{array}{l}\text { COBERTORA } \\
\text { VACIKAL }\end{array}$ & $\begin{array}{l}\text { POPULACAO } \\
\text { DA UPA }\end{array}$ & $\begin{array}{l}\text { POPULACAO } \\
\text { ACUUULADA }\end{array}$ \\
\hline 1 & 53 & 450 & 0,95 & 0,126667 & 0,894737 & 1800 & 1800 \\
\hline 2 & 83 & 450 & 0,90 & 0,006667 & 1,000000 & 1800 & 3600 \\
\hline 3 & 3 & 300 & 0,80 & 0,140000 & 0,976190 & 1800 & 5400 \\
\hline 1 & 70 & 300 & 0,90 & 0,110000 & 1,000000 & 1200 & 6600 \\
\hline 5 & 27 & 200 & 0,90 & 0,115000 & 0,956522 & 1000 & 7600 \\
\hline 6 & 26 & 200 & 0,90 & 0.135000 & 1,000000 & 1000 & 8600 \\
\hline 7 & 85 & 600 & 0,85 & 0,108333 & 1,000000 & 2400 & 11000 \\
\hline 8 & 97 & 450 & 0,85 & 0,115556 & 0,942308 & 1575 & 12575 \\
\hline 9 & 17 & 300 & 0,90 & 0.150000 & 0,977778 & 1650 & 14225 \\
\hline 10 & 77 & 200 & 0,95 & 0,130000 & 1,000000 & 800 & 15025 \\
\hline 11 & 78 & 200 & 0.95 & 0.115000 & 1.000000 & 800 & 15825 \\
\hline 12 & 95 & 300 & 0,80 & 0,100000 & 1,000000 & 1050 & 16875 \\
\hline 13 & 80 & 300 & 0.90 & 0.126667 & 0.973684 & 1200 & 18075 \\
\hline 14 & 74 & 600 & 0,85 & 0,116667 & 0,957143 & 2400 & 20475 \\
\hline 15 & 30 & 450 & 0,80 & 0,151111 & 1,000000 & 2250 & 22725 \\
\hline 16 & 48 & 200 & 0,95 & 0,130000 & 0,961538 & 1100 & 23825 \\
\hline 17 & 33 & 200 & 0,85 & 0,155000 & 0,903226 & 900 & 24725 \\
\hline 18 & 13 & 600 & 0,95 & 0,143333 & 0,953488 & 3600 & 28325 \\
\hline 19 & 71 & 300 & 0,95 & 0,133333 & 0,875000 & 1200 & 29525 \\
\hline 20 & 39 & 450 & 0,85 & 0,142222 & 0,875000 & 2025 & 31550 \\
\hline 21 & 96 & 300 & 0,95 & 0,116667 & 0,971429 & 1050 & 32600 \\
\hline 22 & 15 & 200 & 0,85 & 0,175000 & 0,914286 & 1100 & 33700 \\
\hline 23 & 7 & 450 & 0,90 & 0,160000 & 0,972222 & 2700 & 36400 \\
\hline 24 & 98 & 450 & 0,90 & 0,115556 & 1,000000 & 1575 & 37975 \\
\hline 25 & 41 & 600 & 0,95 & 0,118333 & 0,873239 & 2700 & 40675 \\
\hline 26 & 52 & 450 & 0,85 & 0,100000 & 0,977778 & 1800 & 42475 \\
\hline 27 & 84 & 450 & 0,90 & 0,102222 & 0,956522 & 1800 & 44275 \\
\hline 28 & 68 & 200 & 0,95 & 0,105000 & 0,952381 & 800 & 45075 \\
\hline 29 & 23 & 450 & 0,95 & 0,146667 & 0,981819 & 2475 & 47550 \\
\hline 30 & 92 & 600 & 0,90 & 0,098333 & 0,881356 & 2100 & 49650 \\
\hline 31 & 58 & 200 & 0,90 & 0,135000 & 0,888889 & 800 & 50450 \\
\hline 32 & 36 & 300 & 0,95 & 0,110000 & 0,969697 & 1350 & 51800 \\
\hline 33 & 32 & 600 & 0,90 & 0,170000 & 0,980392 & 3000 & 54800 \\
\hline 34 & 18 & 300 & 0,85 & 0,170000 & 1,000000 & 1650 & 56450 \\
\hline 35 & 29 & 300 & 0,85 & 0,140000 & 0,976190 & 1500 & 57950 \\
\hline 36 & 34 & 300 & 0,85 & 0,150000 & 0,911111 & 1350 & 59300 \\
\hline 37 & 6 & 300 & 0,95 & 0,173333 & 0,980769 & 1800 & 61100 \\
\hline 38 & 69 & 300 & 0,90 & 0,113333 & 0,911765 & 1200 & 62300 \\
\hline 39 & 42 & 200 & 0,90 & 0,115000 & 1,000000 & 900 & 63200 \\
\hline 40 & 63 & 450 & 0,80 & 0,115556 & 0,961538 & 1800 & 65000 \\
\hline$\$ 1$ & 89 & 450 & 0,90 & 0,113333 & 0,882353 & 1575 & 66575 \\
\hline 12 & 65 & 450 & 0,95 & 0,115556 & 1,000000 & 1800 & 68375 \\
\hline 43 & 5 & 300 & 0,95 & 0,170000 & 1,000000 & 1800 & 70175 \\
\hline 14 & 25 & 200 & 0,90 & 0,130000 & 1,000000 & 1000 & 71175 \\
\hline 45 & 82 & 450 & 0,85 & 0,113333 & 0,960784 & 1800 & 72975 \\
\hline 46 & 61 & 300 & 0,95 & 0,086667 & 1,000000 & 1200 & 74175 \\
\hline 47 & 57 & 200 & 0,90 & 0,110000 & 0,954545 & 800 & 74975 \\
\hline 48 & 62 & 450 & 0,80 & 0,137778 & 0,983871 & 1800 & 76775 \\
\hline 49 & 46 & 450 & 0,95 & 0,146667 & 0,984849 & 2025 & 78800 \\
\hline 50 & 73 & 450 & 0,90 & 0,151111 & 0,882353 & 1800 & 80600 \\
\hline
\end{tabular}


QUADRO 5.4 - POPULACAO SIRULADA 4 (Conclusao)

\begin{tabular}{|c|c|c|c|c|c|c|c|}
\hline $\begin{array}{l}\text { MOLERO DE } \\
\text { ORDEH }\end{array}$ & $\begin{array}{l}\text { RWEERO DA } \\
\text { OPA }\end{array}$ & $\begin{array}{l}\text { MUERO DE } \\
\text { DOHICILIOS }\end{array}$ & $\begin{array}{l}\text { TAXA DE } \\
\text { RESPOSTA }\end{array}$ & $\begin{array}{l}\text { DENS. INTRA. } \\
\text { DE CRIANCAS }\end{array}$ & $\begin{array}{l}\text { COBERTURA } \\
\text { DACIIIAL }\end{array}$ & $\begin{array}{l}\text { POPULACAO } \\
\text { DA UPA }\end{array}$ & $\begin{array}{l}\text { POPULACAO } \\
\text { ACUAULADA }\end{array}$ \\
\hline 51 & 75 & 200 & 0,90 & 0,105000 & 1,000000 & 800 & 81400 \\
\hline 52 & 51 & 450 & 0,80 & 0,115556 & 0,884615 & 1800 & 83200 \\
\hline 53 & 59 & 200 & 0,95 & 0,120000 & 0,916667 & 800 & 84000 \\
\hline 54 & 49 & 300 & 0,90 & 0,103333 & 0,967742 & 1200 & 85200 \\
\hline .55 & 1 & 200 & 0,95 & 0,110000 & 0,954545 & 1200 & 86400 \\
\hline 56 & 60 & 300 & 0,95 & 0,116667 & 0,971429 & 1200 & 87600 \\
\hline 57 & 9 & 600 & 0,85 & 0,163333 & 1,000000 & 3600 & 91200 \\
\hline 58 & 72 & 450 & 0,85 & 0,122222 & 0,890909 & 1800 & 93000 \\
\hline 59 & 21 & 450 & 0,85 & 0,157778 & 0,985916 & 2475 & 95475 \\
\hline 60 & 55 & 600 & 0,90 & 0,106667 & 0,921875 & 2400 & 97875 \\
\hline 61 & 76 & 200 & 0,95 & 0,125000 & 1,000000 & 800 & 98675 \\
\hline 62 & 56 & 200 & 0,90 & 0,150000 & 0,966667 & 800 & 99475 \\
\hline 63 & 8 & 450 & 0,95 & 0,168889 & 0,986842 & 2700 & 102175 \\
\hline 64 & 91 & 600 & 0,85 & 0,091667 & 0,909091 & 2100 & 104275 \\
\hline 65 & 64 & 450 & 0,90 & 0,111111 & 1,000000 & 1800 & 106075 \\
\hline 66 & 43 & 450 & 0,85 & 0,151111 & 0,985294 & 2025 & 108100 \\
\hline 67 & 28 & 300 & 0,85 & 0,143333 & 1,000000 & 1500 & 109600 \\
\hline 68 & 93 & 200 & 0,95 & 0,175000 & 1,000000 & 1000 & 110600 \\
\hline 69 & 11 & 600 & 0,90 & 0,161667 & 0,979381 & 3600 & 114200 \\
\hline 70 & 50 & 300 & 0,95 & 0,133333 & 0,850000 & 1200 & 115400 \\
\hline 71 & 16 & 200 & 0,95 & 0,180000 & 1,000000 & 1100 & 116500 \\
\hline 72 & 99 & 600 & 0,80 & 0,115000 & 1,000000 & 2100 & 118600 \\
\hline 73 & 54 & 600 & 0,85 & 0,105000 & 0,873016 & 2400 & 121000 \\
\hline 74 & 88 & 300 & 0,95 & 0,113333 & 0,911765 & 1050 & 122050 \\
\hline 75 & 2 & 300 & 0,80 & 0,143333 & 1,000000 & 1800 & 123850 \\
\hline 76 & 10 & 600 & 0,90 & 0,195000 & 0,982906 & 3600 & 127450 \\
\hline 77 & 44 & 300 & 0,95 & 0,160000 & 0,895833 & 1350 & 128800 \\
\hline 78 & 45 & 300 & 0,95 & 0,130000 & 1,000000 & 1350 & 130150 \\
\hline 79 & 35 & 300 & 0,85 & 0,130000 & 1,000000 & 1350 & 131500 \\
\hline 80 & 38 & 450 & 0,80 & 0,120000 & 0,833333 & 2025 & 133525 \\
\hline 81 & 94 & 300 & 0,80 & 0,120000 & 1,000000 & 1050 & 134575 \\
\hline 82 & 22 & 450 & 0,85 & 0,140000 & 0,936508 & 2475 & 137050 \\
\hline 83 & 31 & 450 & 0,95 & 0,146667 & 0,969697 & 2250 & 139300 \\
\hline 84 & 4 & 300 & 0,90 & 0,173333 & 1,000000 & 1800 & 141100 \\
\hline 85 & 66 & 600 & 0,85 & 0,120000 & 0,986111 & 2400 & 143500 \\
\hline 86 & 86 & 200 & 0,95 & 0,120000 & 1,000000 & 1200 & 144700 \\
\hline 87 & 100 & 600 & 0,85 & 0,071667 & 0,976744 & 2100 & 146800 \\
\hline 88 & 87 & 300 & 0,90 & 0,136667 & 0,902439 & 1050 & 147850 \\
\hline 89 & 19 & 300 & 0,90 & 0,173333 & 0,980769 & 1650 & 149500 \\
\hline 90 & 12 & 600 & 0,90 & 0,150000 & 0,977778 & 3600 & 153100 \\
\hline 91 & 37 & 300 & 0,95 & 0,146667 & 0,931818 & 1350 & 154450 \\
\hline 92 & 81 & 300 & 0,95 & 0,116667 & 1,000000 & 1200 & 155650 \\
\hline 93 & 14 & 200 & 0,80 & 0,160000 & 0,968750 & 1100 & 156750 \\
\hline 94 & 40 & 600 & 0,90 & 0,126667 & 0,894737 & 2700 & 159450 \\
\hline 95 & 20 & 300 & 0,95 & 0,140000 & 0,952381 & 1650 & 161100 \\
\hline 96 & 90 & 450 & 0,90 & 0,084444 & 0,894737 & 1575 & 162675 \\
\hline 97 & 47 & 600 & 0,95 & 0,121667 & 0,986301 & 2700 & 165375 \\
\hline 98 & 79 & 300 & 0,85 & 0,130000 & 0,974359 & 1200 & 166575 \\
\hline 99 & 67 & 200 & 0,85 & 0,125000 & 0,760000 & 800 & 167375 \\
\hline 100 & 24 & 600 & 0,85 & 0,131667 & 0,987342 & 3295 & 170670 \\
\hline
\end{tabular}


TABELA 5.1 - DISTRIBOICAO MHOSTRAL DO ESTIMADOR-RAZAO E DA SUA VARIARCIA PARA A POPULACAO I

\begin{tabular}{|c|c|c|c|c|c|c|c|}
\hline \multirow[t]{2}{*}{ MHOSTRAS } & \multirow[t]{2}{*}{ PROBABILIDADE } & \multicolumn{2}{|c|}{ PAI } & \multicolumn{2}{|c|}{$S \& V$} & \multicolumn{2}{|c|}{ SILVA } \\
\hline & & $\mathbf{r}$ & $\operatorname{var}(r)$ & $\mathbf{r}$ & $\operatorname{var}(r)$ & $\mathbf{r}$ & $\operatorname{var}(r)$ \\
\hline 1 & 0.001582 & 0.707843 & 0.024735 & 0.714320 & 0.020956 & 0.713079 & 0.021535 \\
\hline 2 & 0.017226 & 0.713494 & 0.023712 & 0.719746 & 0.020998 & 0.718361 & 0.019962 \\
\hline 3 & 0.008964 & 0.708843 & 0.023235 & 0.715711 & 0.021695 & 0.714626 & 0.019640 \\
\hline 1 & 0.003164 & 0.707557 & 0.023696 & 0.714484 & 0.021625 & 0.713501 & 0.020159 \\
\hline 5 & 0.007382 & 0.714232 & 0.023364 & 0.719965 & 0.020618 & 0.719030 & 0.020195 \\
\hline 6 & 0.030936 & 0.716713 & 0.023369 & 0.720064 & 0.020553 & 0.719212 & 0.020004 \\
\hline 7 & 0.004570 & 0.714100 & 0.024170 & 0.716154 & 0.021586 & 0.715104 & 0.020166 \\
\hline 8 & 0.016523 & 0.710232 & 0.024029 & 0.712418 & 0.021176 & 0.711376 & 0.019884 \\
\hline 9 & 0.014941 & 0.710661 & 0.023994 & 0.713476 & 0.020423 & 0.712519 & 0.019569 \\
\hline 10 & 0.003867 & 0.711136 & 0.023268 & 0.715398 & 0.020864 & 0.714506 & 0.019470 \\
\hline 11 & 0.005624 & 0.710842 & 0.023154 & 0.716091 & 0.021249 & 0.715837 & 0.018227 \\
\hline 12 & 0.034100 & 0.709222 & 0.023035 & 0.715689 & 0.021360 & 0.715440 & 0.018764 \\
\hline 13 & 0.000878 & 0.711273 & 0.024169 & 0.714014 & 0.021494 & 0.713258 & 0.018760 \\
\hline 19 & 0.010370 & 0.709420 & 0.021069 & 0.711984 & 0.021518 & 0.711563 & 0.019387 \\
\hline 15 & 0.007558 & 0.716809 & 0.023575 & 0.718818 & 0.021775 & 0.718432 & 0.019039 \\
\hline 16 & 0.009492 & 0.722483 & 0.024274 & 0.723206 & 0.020966 & 0.722662 & 0.018577 \\
\hline 17 & 0.001757 & 0.710870 & 0.025316 & 0.712493 & 0.021371 & 0.712122 & 0.020259 \\
\hline 18 & 0.007382 & 0.707713 & 0.025489 & 0.711991 & 0.021408 & 0.711507 & 0.020937 \\
\hline 19 & 0.000878 & 0.709497 & 0.023999 & 0.709302 & 0.020415 & 0.707695 & 0.020172 \\
\hline 20 & 0.000703 & 0.716091 & 0.023453 & 0.714705 & 0.020282 & 0.713342 & 0.021069 \\
\hline 21 & 0.001933 & 0.709318 & 0.023341 & 0.708179 & 0.020729 & 0.706882 & 0.021554 \\
\hline 22 & 0.014413 & 0.708695 & 0.023369 & 0.707315 & 0.020389 & 0.706527 & 0.020856 \\
\hline 23 & 0.006152 & 0.712704 & 0.023500 & 0.710716 & 0.019832 & 0.710145 & 0.020746 \\
\hline 24 & 0.013710 & 0.701908 & 0.024193 & 0.700583 & 0.020579 & 0.700538 & 0.020910 \\
\hline 25 & 0.041132 & 0.702163 & 0.024702 & 0.699127 & 0.020288 & 0.699232 & 0.020934 \\
\hline 26 & 0.003867 & 0.707274 & 0.024416 & 0.704697 & 0.019632 & 0.704903 & 0.020766 \\
\hline 27 & 0.009667 & 0.711612 & 0.023635 & 0.706545 & 0.019587 & 0.706488 & 0.021883 \\
\hline 28 & 0.001406 & 0.717126 & 0.023892 & 0.712383 & 0.018656 & 0.712383 & 0.022417 \\
\hline 29 & 0.004570 & 0.714119 & 0.022911 & 0.710826 & 0.017790 & 0.710873 & 0.022016 \\
\hline 30 & 0.023554 & 0.725478 & 0.022512 & 0.720534 & 0.018129 & 0.720955 & 0.021953 \\
\hline 31 & 0.008085 & 0.717893 & 0.021906 & 0.714020 & 0.018463 & 0.714173 & 0.022828 \\
\hline 32 & 0.001106 & 0.720528 & 0.021746 & 0.716088 & 0.018776 & 0.716212 & 0.023422 \\
\hline 33 & 0.011425 & 0.727461 & 0.020830 & 0.722013 & 0.018391 & 0.722474 & 0.023546 \\
\hline 34 & 0.005976 & 0.725932 & 0.020643 & 0.721020 & 0.018610 & 0.720983 & 0.023406 \\
\hline 35 & 0.000703 & 0.723785 & 0.021596 & 0.719118 & 0.017621 & 0.718607 & 0.023475 \\
\hline 36 & 0.008964 & 0.722125 & 0.021303 & 0.716040 & 0.017404 & 0.715550 & 0.023525 \\
\hline 37 & 0.001230 & 0.724268 & 0.020426 & 0.718411 & 0.016929 & 0.717839 & 0.022522 \\
\hline 38 & 0.001230 & 0.723893 & 0.020380 & 0.717572 & 0.017414 & 0.717371 & 0.023290 \\
\hline 39 & 0.010898 & 0.720485 & 0.020636 & 0.713612 & 0.018180 & 0.713284 & 0.023155 \\
\hline 40 & 0.001582 & 0.727597 & 0.020607 & 0.721463 & 0.017845 & 0.721094 & 0.022535 \\
\hline 41 & 0.038319 & 0.727061 & 0.019515 & 0.723822 & 0.017592 & 0.723600 & 0.022661 \\
\hline 42 & 0.000175 & 0.723434 & 0.019543 & 0.720554 & 0.018606 & 0.720449 & 0.022207 \\
\hline 43 & 0.004570 & 0.733210 & 0.018519 & 0.730403 & 0.018472 & 0.730305 & 0.021297 \\
\hline 44 & 0.017929 & 0.742506 & 0.018078 & 0.739237 & 0.017858 & 0.739157 & 0.021322 \\
\hline 45 & 0.002812 & 0.740305 & 0.018529 & 0.737201 & 0.018003 & 0.737293 & 0.021987 \\
\hline 46 & 0.001933 & 0.734537 & 0.018719 & 0.732804 & 0.018627 & 0.733048 & 0.021948 \\
\hline 47 & 0.009492 & 0.734068 & 0.018523 & 0.732380 & 0.019389 & 0.733461 & 0.022150 \\
\hline 48 & 0.005976 & 0.734893 & 0.017922 & 0.734965 & 0.019651 & 0.736223 & 0.022387 \\
\hline 49 & 0.012656 & 0.718967 & 0.018650 & 0.720248 & 0.020433 & 0.721440 & 0.023271 \\
\hline 50 & 0.012480 & 0.712659 & 0.019390 & 0.716035 & 0.020791 & 0.717294 & 0.022103 \\
\hline
\end{tabular}


TABEA 5.1 - DISTRIBOICAO ANOSTRAL DO ESTIMADOR-RAZAO E DA SUA PARIAACIA PARA A POPOLACAO I (Conclueso)

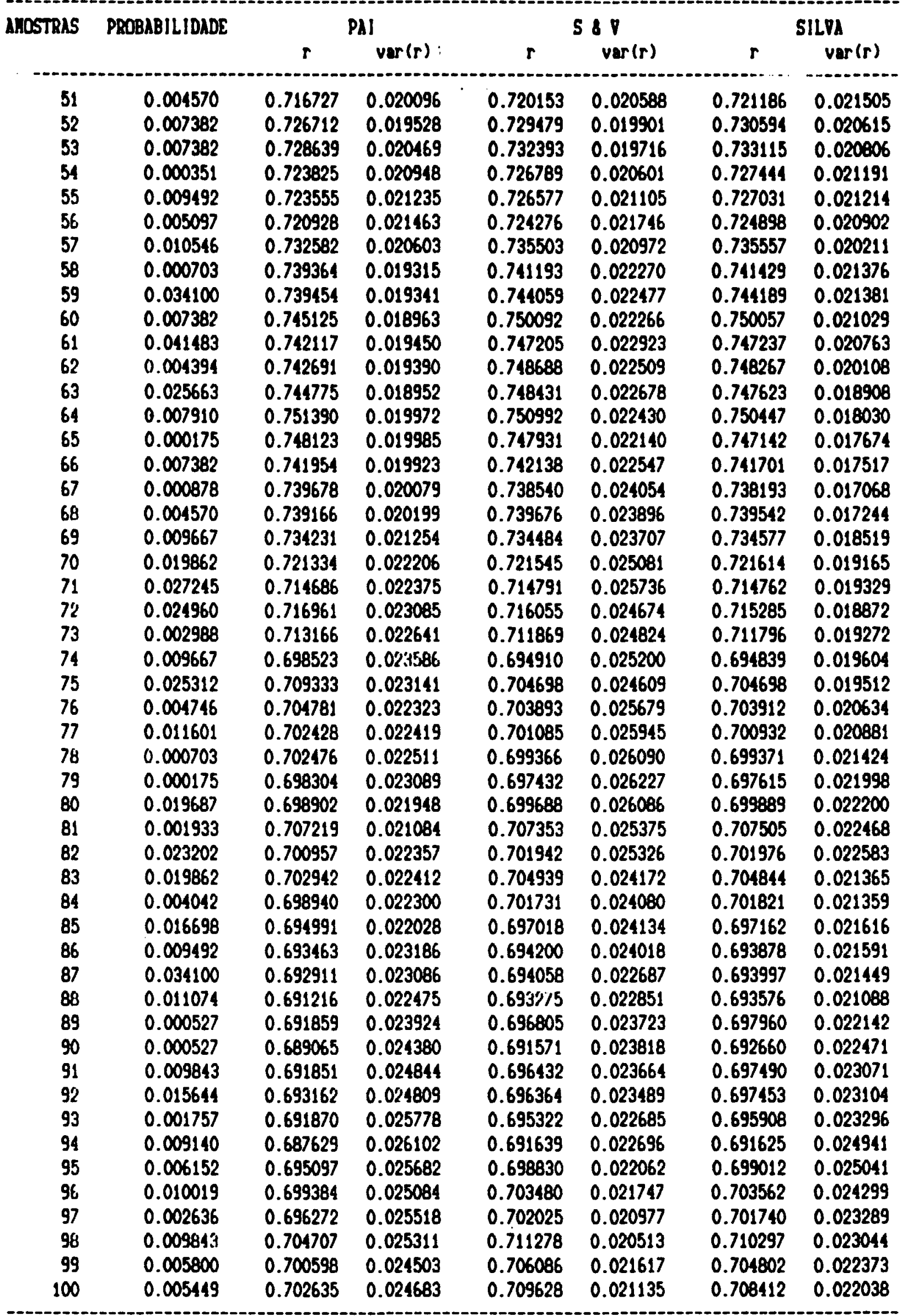

$\begin{array}{lllllll}\text { POPDLACAO } & \text { ESP(r) } & \text { PAR(r) } & \text { ESP(r) } & \operatorname{PAR}(r) & \text { ESP(r) } & \text { VAR(r) }\end{array}$


TABELA 5.2 - DISTRIBUICAO ANOSTRAL DO ESTIMADOR-RAZAO E DA SUA VARIAICIA PARA A POPULACAO 2

\begin{tabular}{|c|c|c|c|c|c|c|c|}
\hline \multirow[t]{2}{*}{ AMOSTRAS } & \multirow[t]{2}{*}{ PROBABILIDADE } & \multicolumn{2}{|c|}{ PAI } & \multicolumn{2}{|c|}{$S \& V$} & \multicolumn{2}{|c|}{ SILVA } \\
\hline & & $\mathbf{r}$ & $\operatorname{var}(r)$ & $\boldsymbol{r}$ & $\operatorname{var}(r)$ & $\boldsymbol{r}$ & $\operatorname{var}(r)$ \\
\hline 1 & 0,001582 & 0,540625 & 0,019380 & 0,539346 & 0,014458 & 0,538773 & 0,016864 \\
\hline 2 & 0,017226 & 0,525828 & 0,019006 & 0,525003 & 0,014717 & 0,524184 & 0,016183 \\
\hline 3 & 0,008964 & 0,503278 & 0,018382 & 0,503787 & 0,014764 & 0,503011 & 0,015896 \\
\hline 4 & 0,003164 & 0,487630 & 0,017744 & 0,488207 & 0,013936 & 0,487431 & 0,016145 \\
\hline 5 & 0,007382 & 0,511390 & 0,017530 & 0,511504 & 0,014165 & 0,510883 & 0,016579 \\
\hline 6 & 0,030936 & 0,513870 & 0,017535 & 0,511603 & 0,014100 & 0,511065 & 0,016389 \\
\hline 7 & 0,004570 & 0,511257 & 0,018336 & 0,507693 & 0,015134 & 0,506957 & 0,016550 \\
\hline 8 & 0,016523 & 0,521156 & 0,018475 & 0,518819 & 0,015168 & 0,518087 & 0,016772 \\
\hline 9 & 0,014941 & 0,522856 & 0,018716 & 0,520643 & 0,015163 & 0,519949 & 0,016702 \\
\hline 10 & 0,003867 & 0,507039 & 0,018024 & 0,505616 & 0,015618 & 0,504982 & 0,016903 \\
\hline 11 & 0,005624 & 0,506745 & 0,017910 & 0,506310 & 0,016003 & 0,506313 & 0,015660 \\
\hline 12 & 0,034100 & 0,505124 & 0,017791 & 0,506100 & 0,016114 & 0,505916 & 0,016197 \\
\hline 13 & 0,000878 & 0,507176 & 0,018925 & 0,504232 & 0,016248 & 0,503734 & 0,016193 \\
\hline 14 & 0,010370 & 0,505323 & 0,018825 & 0,502202 & 0,016272 & 0,502039 & 0,016821 \\
\hline 15 & 0,007558 & 0,489885 & 0,017664 & 0,487726 & 0,016158 & 0,487588 & 0,016003 \\
\hline 16 & 0,009492 & 0,495558 & 0,018363 & 0,492114 & 0,015350 & 0,491818 & 0,015541 \\
\hline 17 & 0,001757 & 0,483945 & 0,019405 & 0,481401 & 0,015755 & 0,481278 & 0,017223 \\
\hline 18 & 0,007382 & 0,480789 & 0,019578 & 0,480899 & 0,015792 & 0,480663 & 0,017901 \\
\hline 19 & 0,000878 & 0,499874 & 0,019743 & 0,499187 & 0,015881 & 0,498581 & 0,018000 \\
\hline 20 & 0,000703 & 0,522450 & 0,020176 & 0,522423 & 0,016212 & 0,521418 & 0,019006 \\
\hline 21. & 0,001933 & 0,535444 & 0,019528 & 0,535780 & 0,016553 & 0,534896 & 0,019617 \\
\hline 22 & 0,014413 & 0,534821 & 0,019555 & 0,534917 & 0,016213 & 0,534541 & 0,018919 \\
\hline 23 & 0,006152 & 0,538831 & 0,019687 & 0,538318 & 0,015656 & 0,538159 & 0,018809 \\
\hline 24 & 0,013710 & 0,514386 & 0,019205 & 0,514203 & 0,015529 & 0,514568 & 0,018646 \\
\hline 25 & 0,041132 & 0,494813 & 0,019710 & 0,495086 & 0,015879 & 0,495265 & 0,018308 \\
\hline 26 & 0,003867 & 0,520372 & 0,018776 & 0,520424 & 0,015006 & 0,520808 & 0,017248 \\
\hline 27 & 0,009667 & 0,524709 & 0,017995 & 0,522272 & 0,014961 & 0,522392 & 0,018365 \\
\hline 28 & 0,001406 & 0,527043 & 0,017934 & 0,522970 & 0,015410 & 0,522934 & 0,018178 \\
\hline 29 & 0,004570 & 0,524035 & 0,016953 & 0,521412 & 0,014544 & 0,521425 & 0,017778 \\
\hline 30 & 0,023554 & 0,512540 & 0,016533 & 0,508606 & 0,014322 & 0,508628 & 0,017248 \\
\hline 31 & 0,008085 & 0,504956 & 0,015927 & 0,502092 & 0,014656 & 0,501846 & 0,018123 \\
\hline 32 & 0,001406 & 0,507590 & 0,015767 & 0,504160 & 0,014969 & 0,503885 & 0,018717 \\
\hline 33 & 0,011425 & 0,528885 & 0,015950 & 0,524437 & 0,015341 & 0,524601 & 0,019112 \\
\hline 34 & 0,005976 & 0,527356 & 0,015764 & 0,523445 & 0,015559 & 0,523110 & 0,018972 \\
\hline 35 & 0,000703 & 0,531817 & 0,015953 & 0,527110 & 0,015583 & 0,526944 & 0,019021 \\
\hline 36 & 0,008964 & 0,540061 & 0,016633 & 0,534317 & 0,017160 & 0,534564 & 0,020154 \\
\hline 37 & 0,001230 & 0,542204 & 0,015756 & 0,536688 & 0,016684 & 0,5 & 0,019151 \\
\hline 38 & 0,001230 & 0,527292 & 0,015414 & 0,521711 & 0,016289 & 0,521989 & 0,018648 \\
\hline 39 & 0,010898 & 0,503722 & 0,015589 & 0,498792 & 0,016510 & 0,499217 & 0,018190 \\
\hline 40 & 0,001582 & 0,531114 & 0,015931 & 0,526660 & 0,016567 & 0,526850 & 0,018250 \\
\hline 41 & 0,038319 & 0,506738 & 0,014560 & 0,505611 & 0,015887 & 0,506250 & 0,017960 \\
\hline 42 & 0,000175 & 0,486027 & 0,014469 & 0,484527 & 0,015665 & 0,485177 & 0,017108 \\
\hline 43 & 0,004570 & 0,512079 & 0,014605 & 0,511046 & 0,015509 & 0,511643 & 0,017198 \\
\hline 44 & 0,017929 & 0,535000 & 0,014912 & 0,534429 & 0,015529 & 0,534822 & 0,017919 \\
\hline 45 & 0,002812 & 0,515016 & 0,015054 & 0,514289 & 0,015276 & 0,514737 & 0,017947 \\
\hline 46 & 0,001933 & 0,494665 & 0,015025 & 0,495265 & 0,015515 & 0,496266 & 0,017600 \\
\hline 47 & 0,009492 & 0,494195 & 0,014829 & 0,494841 & 0,016278 & 0,496679 & 0,017810 \\
\hline 48 & 0,005976 & 0,514849 & 0,014232 & 0,515087 & 0,015900 & 0,517439 & 0,018410 \\
\hline 49 & 0,012656 & 0,485157 & 0,014679 & 0,485508 & 0,016238 & 0,487797 & 0,018790 \\
\hline 50 & 0,012480 & 0,462629 & 0,014818 & 0,464128 & 0,016205 & 0,466553 & 0,017338 \\
\hline
\end{tabular}




\begin{tabular}{|c|c|c|c|c|c|c|c|}
\hline \multirow[t]{2}{*}{ M,OSTRAS } & \multirow[t]{2}{*}{ PROBABILIDADE } & \multicolumn{2}{|c|}{ PAI } & \multicolumn{2}{|c|}{$5 \& V$} & \multicolumn{2}{|c|}{ SILVA } \\
\hline & & $\boldsymbol{r}$ & $\operatorname{var}(r)$ & $r$ & $\operatorname{var}(r)$ & $r$ & $\operatorname{var}(r)$ \\
\hline $\begin{array}{l}51 \\
52\end{array}$ & $\begin{array}{l}0,004570 \\
0,00738 ?\end{array}$ & $\begin{array}{l}0,466697 \\
0,476682\end{array}$ & $\begin{array}{l}0,015524 \\
0.014956\end{array}$ & $\begin{array}{l}0,468246 \\
0,477571\end{array}$ & $\begin{array}{l}0,016001 \\
0.015315\end{array}$ & $\begin{array}{l}0,470445 \\
0.479853\end{array}$ & $\begin{array}{l}0,016740 \\
0.015850\end{array}$ \\
\hline 53 & 0,007382 & 0,501463 & 0,015918 & 0,503000 & 0,015691 & 0,505252 & 0,016507 \\
\hline 54 & 0,000351 & 0,478176 & 0,016011 & 0,479376 & 0,015889 & 0,481496 & 0,016800 \\
\hline 55 & 0,009492 & 0,474087 & 0,015613 & 0,475637 & 0,015721 & 0,477673 & 0,016687 \\
\hline 56 & 0,005097 & 0,471460 & 0,015840 & 0,473336 & 0,016363 & 0,475541 & 0,016374 \\
\hline 57 & 0,010546 & 0,496763 & 0,016155 & 0,498544 & 0,016461 & 0,500183 & 0,016010 \\
\hline 58 & 0,000703 & 0,514836 & 0,015776 & 0,515848 & 0,017397 & 0,517283 & 0,017161 \\
\hline 59 & 0,034100 & 0,527849 & 0,015496 & 0,529740 & 0,016181 & 0,530687 & 0,016551 \\
\hline 60 & 0,007382 & 0,533520 & 0,015119 & 0,535773 & 0,015970 & 0,536551 & 0,016199 \\
\hline 61 & 0,041483 & 0,554351 & 0,015884 & 0,556293 & 0,017051 & 0,556840 & 0,016350 \\
\hline 62 & 0,004394 & 0,554926 & 0,015825 & 0,557777 & 0,016640 & 0,557870 & 0,015695 \\
\hline 63 & 0,025663 & 0,541396 & 0,015024 & 0,543523 & 0,016723 & 0,543402 & 0,014796 \\
\hline 64 & 0,007910 & 0,548010 & 0,016043 & 0,546084 & 0,016474 & 0,546225 & 0,013919 \\
\hline 65 & 0,000175 & 0,542671 & 0,015683 & 0,541435 & 0,016412 & 0,541374 & 0,013176 \\
\hline 66 & 0,007382 & 0,515909 & 0,015290 & 0,515275 & 0,015823 & 0,515247 & 0,012390 \\
\hline 67 & 0,000878 & 0,513633 & 0,015446 & 0,511677 & 0,017330 & 0,511739 & 0,011941 \\
\hline 68 & 0,004570 & 0,513121 & 0,015566 & 0,512813 & 0,017172 & 0,513088 & 0,012116 \\
\hline 69 & 0,009667 & 0,508186 & 0,016621 & 0,507621 & 0,016983 & 0,508123 & 0,013391 \\
\hline 70 & 0,019862 & 0,478984 & 0,017022 & 0,478236 & 0,017304 & 0,478747 & 0,013745 \\
\hline 71 & 0,027245 & 0,472335 & 0,017191 & 0,471482 & 0,017959 & 0,471896 & 0,013908 \\
\hline 72 & 0,024960 & 0,474610 & 0,017901 & 0,472746 & 0,016897 & 0,472419 & 0,013452 \\
\hline 73 & 0,002988 & 0,470816 & 0,017457 & 0,468559 & 0,017047 & 0,468929 & 0,013852 \\
\hline 74 & 0,009667 & 0,442387 & 0,017452 & 0,439554 & 0,017215 & 0,439890 & 0,013821 \\
\hline 75 & 0,025312 & 0,467780 & 0,017227 & 0,463968 & 0,017008 & 0,463975 & 0,014028 \\
\hline 76 & 0,004746 & 0,463229 & 0,016409 & 0,463164 & 0,018078 & 0,463189 & 0,015150 \\
\hline 77 & 0,011601 & 0,463469 & 0,016358 & 0,462974 & 0,018101 & 0,462981 & 0,014985 \\
\hline 78 & 0,000703 & 0,463517 & 0,016450 & 0,161256 & 0,018246 & 0,461420 & 0,015528 \\
\hline 79 & 0,000175 & 0,479508 & 0,017109 & 0,478279 & 0,018928 & 0,478349 & 0,016426 \\
\hline 80 & 0,019687 & 0,480106 & 0,015968 & 0,480536 & 0,018787 & 0,480623 & 0,016628 \\
\hline 81 & 0,001933 & 0,507276 & 0,016065 & 0,507409 & 0,019051 & 0,507343 & 0,017079 \\
\hline 82 & 0,023202 & 0,501014 & 0,017338 & 0,501998 & 0,019002 & 0,501813 & 0,017194 \\
\hline 83 & 0,019862 & 0,502999 & 0,017393 & 0,504995 & 0,017848 & 0,504682 & 0,015976 \\
\hline 84 & 0,004042 & 0,498997 & 0,017280 & 0,501786 & 0,017757 & 0,501658 & 0,015970 \\
\hline 85 & 0,016698 & 0,495047 & 0,017009 & 0,497074 & 0,017810 & 0,497000 & 0,016227 \\
\hline 86 & 0,009492 & 0,493520 & 0,018167 & 0,494255 & 0,017694 & 0,493715 & 0,016202 \\
\hline 87 & 0,034100 & 0,492968 & 0,018066 & 0,494113 & 0,016364 & 0,493834 & 0,016060 \\
\hline 88 & 0,011074 & 0,511283 & 0,017801 & 0,514393 & 0,016468 & 0,514260 & 0,016418 \\
\hline 89 & 0,000527 & 0,512989 & 0,017934 & 0,515481 & 0,016018 & 0,515414 & 0,016693 \\
\hline 90 & 0,000527 & 0,489915 & 0,018019 & 0,490230 & 0,015721 & 0,490290 & 0,016341 \\
\hline 91 & 0,009843 & 0,490211 & 0,018272 & 0,490468 & 0,015797 & 0,490592 & 0,016304 \\
\hline 92 & 0,015644 & 0,491523 & 0,018237 & 0,490400 & 0,015622 & 0,490555 & 0,016337 \\
\hline 93 & 0,001757 & 0,510824 & 0,019538 & 0,509726 & 0,015813 & 0,509697 & 0,017158 \\
\hline 94 & 0,009140 & 0,506583 & 0,019862 & 0,506043 & 0,015824 & 0,505414 & 0,018803 \\
\hline 95 & 0,006152 & 0,532525 & 0,019829 & 0,531251 & 0,015877 & 0,530886 & 0,018997 \\
\hline .96 & 0,010019 & 0,536812 & 0,019231 & 0,535904 & 0,015563 & 0,535435 & 0,018254 \\
\hline 97 & 0,002636 & 0,517718 & 0,018687 & 0,516616 & 0,014328 & 0,516424 & 0,017135 \\
\hline 98 & 0,009843 & 0,540689 & 0,018776 & 0,540008 & 0,014745 & 0,539375 & 0,018161 \\
\hline 99 & 0,005800 & 0,519750 & 0,017940 & 0,518895 & 0,014529 & 0,518387 & 0,017492 \\
\hline 100 & 0,005449 & 0,517633 & 0,019019 & 0,516550 & 0,014239 & 0,515889 & 0,016730 \\
\hline POPULACAO & & $\operatorname{ESP}(r)$ & $\operatorname{VAB}(r)$ & $\operatorname{ESP}(r)$ & $\operatorname{VAR}(r)$ & $\operatorname{ESP}(r)$ & $\operatorname{VAR}(r)$ \\
\hline & 1,000000 & 0,506092 & 0,017102 & 0,505661 & 0,016222 & 0,505840 & 0,016538 \\
\hline
\end{tabular}


TABELA 5.3 - DISTRIBUICAO MIOSTRAL DO ESTIMADOR-RIZZO E DA SUA VARIAMCIA PARA A POPULACAO 3

\begin{tabular}{|c|c|c|c|c|c|c|c|}
\hline \multirow[t]{2}{*}{ MHOSTRIS } & \multirow[t]{2}{*}{ PROBIBILIDLDE } & \multicolumn{2}{|c|}{ PAI } & \multicolumn{2}{|c|}{$S \& V$} & \multicolumn{2}{|c|}{ SILP } \\
\hline & & $\mathbf{r}$ & $\operatorname{var}(r)$ & $\mathbf{r}$ & $\operatorname{var}(r)$ & $\boldsymbol{r}$ & $\operatorname{var}(r)$ \\
\hline 1 & 0,001582 & 0,496490 & 0,029581 & 0,497974 & 0,026797 & 0,499200 & 0,025961 \\
\hline 2 & 0,017226 & 0,493935 & 0,030569 & 0,495094 & 0,025991 & 0,496775 & 0,024226 \\
\hline 3 & 0,008964 & 0,489730 & 0,029387 & 0,492442 & 0,027235 & 0,494103 & 0,024491 \\
\hline 4 & 0,003164 & 0,482570 & 0,030189 & 0,485833 & 0,027598 & 0,187255 & 0,022540 \\
\hline 5 & 0,007382 & 0,484518 & 0,030461 & 0,487091 & 0,028066 & 0,488614 & 0,022708 \\
\hline 6 & 0,030936 & 0,474613 & 0,029941 & 0,479725 & 0,027901 & 0,480891 & 0,023598 \\
\hline 7 & 0,004570 & 0,170676 & 0,031230 & 0,176915 & 0,028568 & 0,477951 & 0,023566 \\
\hline 8 & 0,016523 & 0,476294 & 0,029722 & 0,483624 & 0,028398 & 0,484762 & 0,023902 \\
\hline 9 & 0,014941 & 0,486119 & 0,031839 & 0,491911 & 0,028434 & 0,492921 & 0,024105 \\
\hline 10 & 0,003867 & 0,483947 & 0,031985 & 0,490579 & 0,028195 & 0,491763 & 0,023821 \\
\hline 11 & 0,005624 & 0,486664 & 0,032238 & 0,492393 & 0,028290 & 0,493165 & 0,024192 \\
\hline 12 & 0,034100 & 0,485510 & 0,032714 & 0,492794 & 0,027406 & 0,493308 & 0,024259 \\
\hline 13 & 0,000878 & 0,487176 & 0,032756 & 0,494021 & 0,027379 & 0,494211 & 0,024860 \\
\hline 14 & 0,010370 & 0,480377 & 0,032980 & 0,489008 & 0,027528 & 0,489047 & 0,025450 \\
\hline 15 & 0,007558 & 0,481376 & 0,032902 & 0,489341 & 0,026301 & 0,488972 & 0,024476 \\
\hline 16 & 0,009492 & 0,488536 & 0,033354 & 0,495123 & 0,026736 & 0,494823 & 0,024490 \\
\hline 17 & 0,001757 & 0,485489 & 0,033739 & 0,492906 & 0,027297 & 0.492545 & 0,024678 \\
\hline 18 & 0,007382 & 0,491649 & 0,034049 & 0,497873 & 0,027805 & 0,497921 & 0,024685 \\
\hline 19 & 0,000878 & 0,495873 & 0,035973 & 0,500857 & 0,027874 & 0,500774 & 0,024660 \\
\hline 20 & 0,000703 & 0,505195 & 0,036665 & 0,509859 & 0,028323 & 0,509665 & 0,025056 \\
\hline 21 & 0,001933 & 0,510495 & 0,034565 & 0,514333 & 0,028580 & 0,513803 & 0,024654 \\
\hline 22 & 0,014413 & 0,514575 & 0,035148 & 0,515905 & 0,027319 & 0,515350 & 0,024953 \\
\hline 23 & 0,006152 & 0,516563 & 0,034601 & 0,518150 & 0,026510 & 0,517447 & 0,025305 \\
\hline 24 & 0,013710 & 0,502273 & 0,035177 & 0,505357 & 0,026468 & 0,504322 & 0,024829 \\
\hline 25 & 0,041132 & 0,501839 & 0,034795 & 0,506333 & 0,026221 & 0,505346 & 0,024715 \\
\hline 26 & 0,003867 & 0,509815 & 0,033412 & 0,513871 & 0,026366 & 0,512799 & 0,024650 \\
\hline 27 & 0,009667 & 0,520655 & 0,032863 & 0,524797 & 0,026023 & 0,523669 & 0,024280 \\
\hline 28 & 0,001406 & 0,520091 & 0,033041 & 0,524681 & 0,026335 & 0,523330 & 0,024339 \\
\hline 29 & 0,004570 & 0,517430 & 0,031365 & 0,522527 & 0,025889 & 0,520336 & 0,025387 \\
\hline 30 & 0,023551 & 0,517756 & 0,031472 & 0,520965 & 0,026316 & 0,518881 & 0,025471 \\
\hline 31 & 0,006085 & 0,513049 & 0,031624 & 0,517496 & 0,025803 & 0,515396 & 0,025245 \\
\hline 32 & 0,001406 & 0,511329 & 0,030965 & 0,515871 & 0,026860 & 0,513499 & 0,025638 \\
\hline 33 & 0,011425 & 0,521511 & 0,030809 & 0,524824 & 0,026407 & 0,522833 & 0,025853 \\
\hline 34 & 0,005976 & 0,517427 & 0,030826 & 0,521839 & 0,025941 & 0,519553 & 0,026254 \\
\hline 35 & 0,000703 & 0,517502 & 0,029565 & 0,520168 & 0,026057 & 0,517875 & 0,026874 \\
\hline 36 & 0,008964 & 0,510359 & 0,029981 & 0,516321 & 0,026103 & 0,513709 & 0,025857 \\
\hline 37 & 0,001230 & 0,515348 & 0,029668 & 0,520369 & 0,026942 & 0,518156 & 0,024994 \\
\hline 38 & 0,001230 & 0,512705 & 0,030173 & 0,517679 & 0,027056 & 0,515767 & 0,025677 \\
\hline 39 & 0,010898 & 0,511633 & 0,029653 & 0,515648 & 0,026875 & 0,514084 & 0,026412 \\
\hline 40 & 0,001582 & 0,517225 & 0,029919 & 0,521346 & 0,027037 & 0,519855 & 0,027075 \\
\hline 41 & 0,038319 & 0,503079 & 0,029150 & 0,509242 & 0,026963 & 0,500065 & 0,026428 \\
\hline 42 & 0,000175 & 0,497966 & 0,028711 & 0,504493 & 0,026055 & 0,503423 & 0,026403 \\
\hline 43 & 0,004570 & 0,509905 & 0,028183 & 0,517147 & 0,025526 & 0,516305 & 0,026503 \\
\hline 44 & 0,017929 & 0,522125 & 0,028178 & 0,527585 & 0,025468 & 0,526761 & 0,026015 \\
\hline 45 & 0,002812 & 0,516716 & 0,028654 & 0,521064 & 0,026643 & 0,521015 & 0,025935 \\
\hline 46 & 0,001933 & 0,509407 & 0,026934 & 0,512706 & 0,026837 & 0,513035 & 0,025855 \\
\hline 47 & 0,009492 & 0,512663 & 0,026482 & 0,515359 & 0,026969 & 0,515416 & 0,025717 \\
\hline 48 & 0,005976 & 0,517129 & 0,026733 & 0,519687 & 0,026860 & 0,519937 & 0,026065 \\
\hline 49 & 0,012656 & 0,506884 & 0,028655 & 0,509928 & 0,027295 & 0,509977 & 0,026512 \\
\hline 50 & 0,012480 & 0,503701 & 0,028260 & 0,505470 & 0,027091 & 0,504823 & 0,027138 \\
\hline
\end{tabular}


TLBEA 5.3 - DISTRIBUICAO AMOSTRL DO ESTIMADOR-BAZAO E DA SUA VABIAMCIA PARA A POPOLACAO 3 (COnCluaso)

\begin{tabular}{|c|c|c|c|c|c|c|c|}
\hline \multirow[t]{2}{*}{ IMOSTRAS } & \multirow[t]{2}{*}{ PROBABILIDADE } & \multicolumn{2}{|c|}{ PAI } & \multicolumn{2}{|c|}{$5 \& V$} & \multicolumn{2}{|c|}{ SILVA } \\
\hline & & $r$ & $\operatorname{var}(r)$ & $\mathbf{r}$ & $\operatorname{var}(r)$ & $\boldsymbol{r}$ & $\operatorname{var}(r)$ \\
\hline 51 & 0,004570 & 0,511516 & 0,028568 & 0,511752 & 0,026955 & 0,511138 & $\begin{array}{l}0,026433 \\
0,025650\end{array}$ \\
\hline $\begin{array}{l}52 \\
53\end{array}$ & $\begin{array}{l}0,007382 \\
0,007382\end{array}$ & $\begin{array}{l}0,520589 \\
0,524269\end{array}$ & $\begin{array}{l}0,027535 \\
0.027651\end{array}$ & $\begin{array}{l}0,519570 \\
0,525484\end{array}$ & $\begin{array}{l}0,026557 \\
0,026156\end{array}$ & $\begin{array}{l}0,519010 \\
0,525133\end{array}$ & $\begin{array}{l}0,025650 \\
0,026104\end{array}$ \\
\hline $\begin{array}{l}53 \\
54\end{array}$ & $\begin{array}{l}0,007382 \\
0,000351\end{array}$ & $\begin{array}{l}0,524269 \\
0,515460\end{array}$ & $\begin{array}{l}0,027651 \\
0,027750\end{array}$ & 0,516209 & 0,025549 & 0,515890 & 0,026695 \\
\hline 55 & 0,009492 & 0,510054 & 0,026532 & 0,511085 & 0,026258 & 0,510661 & 0,026442 \\
\hline 56 & 0,005097 & 0,513562 & 0,026610 & 0,514198 & 0,026227 & 0,513800 & 0,026247 \\
\hline 57 & 0,010546 & 0,526216 & 0,026580 & 0,525604 & 0,026043 & 0,525172 & 0,025796 \\
\hline 58 & 0,000703 & 0,530544 & 0,026006 & 0,531550 & 0,025483 & 0,531496 & 0,026044 \\
\hline 59 & 0,034100 & 0,537648 & 0,025861 & 0,537577 & 0,025477 & 0,537825 & 0,026600 \\
\hline 60 & 0,007382 & 0,539379 & 0,026819 & 0,540878 & 0,025253 & 0,540883 & 0,027602 \\
\hline 61 & 0,041483 & 0,548658 & 0,027310 & 0,548881 & 0,025581 & 0,5488000 & 0,029456 \\
\hline 62 & 0,004394 & 0,550038 & 0,026924 & 0,551243 & 0,025418 & 0,551035 & 0,029347 \\
\hline 63 & 0,025663 & 0,554355 & 0,026627 & 0,554480 & 0,025258 & 0,554505 & 0,029653 \\
\hline 64 & 0,007910 & 0,552771 & 0,025957 & 0,552127 & 0,024794 & 0,552248 & 0,028397 \\
\hline 65 & 0,000175 & 0,549507 & 0,025420 & 0,548580 & 0,024423 & 0,548537 & 0,028679 \\
\hline 66 & 0,007382 & 0,547636 & 0,025718 & 0,546305 & 0,023147 & 0,546548 & 0,028288 \\
\hline 67 & 0,000878 & 0,545257 & 0,026001 & 0,544123 & 0,023405 & 0,544216 & 0,028786 \\
\hline 68 & 0,004570 & 0,542304 & 0,025309 & 0,540948 & 0,023783 & 0,541081 & 0,029216 \\
\hline 69 & 0,009667 & 0,543906 & 0,024530 & 0,541912 & 0,024172 & 0,541859 & 0,030107 \\
\hline 70 & 0,019862 & 0,530924 & 0,024636 & 0,530947 & 0,024362 & 0,531190 & 0,030127 \\
\hline 71 & 0,027245 & 0,527503 & 0,024627 & 0,526230 & 0,024595 & 0,526753 & 0,029877 \\
\hline 72 & 0,024960 & 0,527460 & 0,027238 & 0,525092 & 0,024205 & 0,525799 & 0,029250 \\
\hline 73 & 0,002988 & 0,534968 & 0,026314 & 0,532595 & 0,022931 & 0,533368 & 0,028249 \\
\hline 74 & 0,009667 & 0,532323 & 0,024562 & 0,529023 & 0,022577 & 0,530149 & 0,026297 \\
\hline 75 & 0,025312 & 0,533546 & 0,024456 & 0,531791 & 0,021788 & 0,532428 & 0,026124 \\
\hline 76 & 0,004746 & 0,532512 & 0,025128 & 0,531910 & 0,022225 & 0,532345 & 0,026363 \\
\hline 77 & 0,011601 & 0,524942 & 0,025424 & 0,526071 & 0,022562 & 0,527231 & 0,025438 \\
\hline 78 & 0,000703 & 0,525149 & 0,025598 & 0,527137 & 0,022702 & 0,527851 & 0,027394 \\
\hline 79 & 0,000175 & 0,523981 & 0,026384 & 0,527544 & 0,023142 & 0,527678 & 0,026434 \\
\hline 80 & 0,019687 & 0,515640 & 0,026238 & 0,520807 & 0,023289 & 0,520763 & 0,026288 \\
\hline 81 & 0,001933 & 0,522601 & 0,025577 & 0,526402 & 0,022788 & 0,526221 & 0,027071 \\
\hline 82 & 0,023202 & 0,511101 & 0,025429 & 0,513731 & 0,023073 & 0,513621 & 0,027013 \\
\hline 83 & 0,019862 & 0,513713 & 0,026043 & 0,515236 & 0,022243 & 0,514689 & 0,026101 \\
\hline 84 & 0,004012 & 0,501851 & 0,026598 & 0,502577 & 0,022340 & 0,502160 & 0,025925 \\
\hline 85 & 0,016698 & 0,493136 & 0,026312 & 0,495162 & 0,022991 & 0,494661 & 0,026320 \\
\hline 86 & 0,009492 & 0,484150 & 0,027040 & 0,486042 & 0,024125 & 0,485364 & 0,027134 \\
\hline 87 & 0,034100 & 0,479325 & 0,026846 & 0,481101 & 0,024878 & 0,480506 & 0,027419 \\
\hline 88 & 0,011074 & 0,488968 & 0,026796 & 0,489562 & 0,024439 & 0,489110 & 0,027427 \\
\hline 89 & 0,000527 & 0,490626 & 0,026742 & 0,491693 & 0,024535 & 0,491423 & 0,027281 \\
\hline 90 & 0,000527 & 0,490884 & 0,026672 & 0,489594 & 0,024848 & 0,489410 & 0,027377 \\
\hline 91 & 0,009843 & 0,485423 & 0,027522 & 0,485445 & 0,025840 & 0,484898 & 0,027970 \\
\hline 92 & 0,015644 & 0,492939 & 0,027620 & 0,491326 & 0,025835 & 0,490873 & 0,028480 \\
\hline 93 & 0,001757 & 0,494175 & 0,026893 & 0,492376 & 0,027085 & 0,492563 & 0,030189 \\
\hline 94 & 0,009140 & 0,499287 & 0,027204 & 0,498437 & 0,027334 & 0,499258 & 0,030331 \\
\hline 95 & 0,006152 & 0,506241 & 0,028025 & 0,505557 & 0,027626 & 0,506368 & 0,030478 \\
\hline 96 & 0,010019 & 0,503953 & 0,027917 & 0,503215 & 0,027275 & 0,504479 & 0,028146 \\
\hline 97 & 0,002636 & 0,498844 & 0,027377 & 0,497709 & 0,027293 & 0,499004 & 0,028127 \\
\hline 98 & 0,009843 & 0,499685 & 0,027130 & 0,500231 & 0,028180 & 0,501163 & 0,027918 \\
\hline 99 & 0,005800 & 0,496590 & 0,027608 & 0,497993 & 0,028215 & 0,498776 & 0,027029 \\
\hline 100 & 0,005419 & 0,487838 & 0,029082 & 0,489862 & 0,028143 & 0,490805 & 0,026973 \\
\hline
\end{tabular}

POPULACAO $\quad \operatorname{ESP}(r) \quad \operatorname{VAR}(r) \quad \operatorname{ESP}(r) \quad \operatorname{VAR}(r) \quad$ ESP(r) $\operatorname{VAR}(r)$

$1,000000 \quad 0,510654 \quad 0,028642 \quad 0,512979 \quad 0,025742 \quad 0,512865 \quad 0,026515$


TABELA 5.4 - DISTRIBUICAO AMOSTRAL DO ESTIIADOR-RAZAO E DA SUA VARIAMCIA PARA A POPULACAO 4

\begin{tabular}{|c|c|c|c|c|c|c|c|}
\hline \multirow[t]{2}{*}{ A.OSTRRS } & \multirow[t]{2}{*}{ PROBABILIDADE } & \multicolumn{2}{|c|}{ PAI } & \multicolumn{2}{|c|}{$5 \& V$} & \multicolumn{2}{|c|}{ SILVA } \\
\hline & & $r$ & $\operatorname{var}(r)$ & $r$ & $\operatorname{var}(r)$ & $r$ & $\operatorname{var}(r)$ \\
\hline 1 & 0,001582 & 0,951205 & 0,006718 & 0,954494 & 0,001712 & 0,955089 & 0,004087 \\
\hline 2 & 0,017226 & 0,951647 & 0,006664 & 0,955111 & 0,004635 & 0,955703 & 0,001021 \\
\hline 3 & 0,008964 & 0,951647 & 0,006664 & 0,955111 & 0,004635 & 0,955703 & 0,004021 \\
\hline 4 & 0,003164 & 0,953003 & 0,006153 & 0,956424 & 0,004619 & 0,956843 & 0,003925 \\
\hline 5 & 0,007382 & 0,948080 & 0,006743 & 0,952181 & 0,004870 & 0,952659 & 0,004319 \\
\hline 6 & 0,030936 & 0,948000 & 0,006743 & 0,952181 & 0,004870 & 0,952659 & 0,004319 \\
\hline 7 & 0,004570 & 0,948596 & 0,006677 & 0,952679 & 0,004806 & 0,953106 & 0,004271 \\
\hline B & 0,016523 & 0,944314 & 0,006982 & 0,947431 & 0,004884 & 0,948043 & 0,004620 \\
\hline 9 & 0,014941 & 0,946006 & 0,006698 & 0,948218 & 0,004838 & 0,948794 & 0,004573 \\
\hline 10 & 0,003867 & 0,945450 & 0,006751 & 0,948516 & 0,004805 & 0,949134 & 0,004533 \\
\hline 11 & 0,005624 & 0,944964 & 0,006813 & 47711 & 0,004897 & 0,948321 & 0,004648 \\
\hline 12 & 0,034100 & 0,939506 & 0,007097 & 0,942705 & 0,005625 & 0,942978 & 0,005051 \\
\hline 13 & 0,000878 & 0,941752 & 0,006933 & 0,945031 & 0,005470 & 0,945354 & 0,004719 \\
\hline 14 & 0,010370 & 0,945555 & 0,006713 & 0,948587 & 0,005010 & 0,948901 & 0,004517 \\
\hline 15 & 0,007558 & 0,945555 & 0,006713 & 0,948587 & 0,005010 & 0,948901 & 0,004517 \\
\hline 16 & 0,009492 & 0,950695 & 0,005918 & 0,953040 & 0,004177 & 0,953388 & 0,004338 \\
\hline 17 & 0,001757 & 0,950819 & 0,005905 & 0,953348 & 0,004448 & 0,953639 & 0,004355 \\
\hline 18 & 0,007382 & 0,950279 & 0,005973 & 0,952694 & 0,004528 & 0,953025 & 0,004420 \\
\hline 19 & 0,000878 & 0,947281 & 0,006241 & 0,949354 & 0,004852 & 0,949823 & 0,004704 \\
\hline 20 & 0,000703 & 0,947787 & 0,006203 & 0,949762 & 0,004823 & 0,950147 & 0,004709 \\
\hline 21 & 0,001933 & 0,944502 & 0,006492 & 0,946918 & 0,004943 & 0,947499 & 0,004893 \\
\hline 22 & 0,014413 & 0,944807 & 0,006415 & 0,947629 & 0,005272 & 0,948165 & 0,005488 \\
\hline 23 & 0,006152 & 0,947522 & 0,005618 & 0,950010 & 0,005102 & 0,950618 & 0,005259 \\
\hline 24 & 0,013710 & 0,949423 & 0,005552 & 0,951339 & 0,004923 & 0,951842 & 0,005109 \\
\hline 25 & 0,041132 & 0,949423 & 0,005552 & 0,951339 & 0,004923 & 0,951842 & 0,005109 \\
\hline 26 & 0,003867 & 0,949423 & 0,005552 & 0,951339 & 0,001923 & 0,951812 & 0,005109 \\
\hline 27 & 0,009667 & 0,951754 & 0,004954 & 0,952619 & 0,004492 & 0,953143 & 0,004749 \\
\hline 28 & 0,001406 & 0,951930 & 0,004932 & 0,952933 & 0,004458 & 0,953460 & 0,004733 \\
\hline 29 & 0,004570 & 0,946858 & 0,005042 & 0,947374 & 0,005013 & 0,947934 & 0,005340 \\
\hline 30 & 0,023554 & 0,951593 & 0,004679 & 0,952969 & 0,004896 & 0,953357 & 0,004959 \\
\hline 31 & 0,000085 & 0,947702 & 0,004905 & 0,949069 & 0,005357 & 0,949338 & 0,005386 \\
\hline 32 & 0,001406 & 0,951597 & 0,004275 & 0,953065 & 0,004887 & 0,953260 & 0,004857 \\
\hline 33 & 0,011425 & 0,951894 & 0,004265 & 0,952815 & 0,004910 & 0,953231 & 0,005168 \\
\hline 34 & 0,005976 & 0,956234 & 0,003694 & 0,956486 & 0,004654 & 0,956788 & 0,004615 \\
\hline 35 & 0,000703 & 0,956234 & 0,003694 & 0,956486 & 0,004654 & 0,956788 & 0,004615 \\
\hline 36 & 0,008964 & 0,956234 & 0,003694 & 0,956486 & 0,004654 & 0,956788 & 0,004615 \\
\hline 37 & 0,001230 & 0,955115 & 0,003816 & 0,955336 & 0,004778 & 0,955670 & 0,004691 \\
\hline 38 & 0,001230 & 0,958005 & 0,003628 & 0,957855 & 0,004814 & 0,958010 & 0,004538 \\
\hline 39 & 0,010898 & 0,962928 & 0,003038 & 0,962098 & 0,004563 & 0,962194 & 0,004144 \\
\hline 40 & 0,001582 & 0,960112 & 0,003175 & 0,959539 & 0,004810 & 0,959648 & 0,004918 \\
\hline 41 & 0,038319 & 0,958481 & 0,003324 & 0,958171 & 0,004946 & 0,958401 & 0,005013 \\
\hline 42 & 0,000175 & 0,959689 & 0,003205 & & 0,004828 & 0,959484 & 0,004888 \\
\hline 43 & 0,004570 & 0,960642 & 0,002963 & 0,960262 & 0,004727 & 0,960402 & 0,004827 \\
\hline 41 & 0,017929 & 0,957982 & 0,003233 & 0,957101 & 0,004986 & 0,957255 & 0,004988 \\
\hline 45 & 0,002812 & 0,962760 & 0,003163 & 0,962152 & 0,004495 & 0,962302 & 0,004436 \\
\hline 46 & 0,001933 & 0,965236 & 0,002962 & 0,964866 & 0,004229 & 0,964749 & 0,004213 \\
\hline 47 & 0,009492 & 0,961036 & 0,003276 & 0,961477 & 0,004678 & 0,961252 & 0,004487 \\
\hline 48 & 0,005976 & 0,960409 & 0,003354 & 0,960643 & 0,004776 & 0,960332 & 0,004692 \\
\hline 49 & 0,012656 & 0,959957 & 0,003413 & 0,960296 & 0,004816 & 0,959973 & 0,004723 \\
\hline 50 & 0,012480 & 0,962683 & 0,003115 & 0,963239 & 0,004626 & 0,962882 & 0,004589 \\
\hline
\end{tabular}


TLBELA 5.4 - DISTRIBUICAO MSOSTRAL dO ESTIMDOR-PRZAO E DA SUA VARIAMCLI PARA A POPOLACAO \& (Conclusao)

\begin{tabular}{|c|c|c|c|c|c|c|c|}
\hline \multirow[t]{2}{*}{ M.OSTRAS } & \multirow[t]{2}{*}{ PROBABILIDLDE } & \multicolumn{2}{|c|}{ PAI } & \multicolumn{2}{|c|}{$5 \& 7$} & \multicolumn{2}{|c|}{ SILV } \\
\hline & & $\boldsymbol{r}$ & $\operatorname{var}(r)$ & $r$ & $\operatorname{var}(r)$ & $r$ & $\operatorname{var}(r)$ \\
\hline 51 & 0,004570 & 0,961794 & & & 0,004673 & 0,962143 & 0,004650 \\
\hline 52 & 0,007382 & 0,967252 & 0,002960 & 0,967524 & 0,003945 & 0,967488 & 0,004247 \\
\hline 53 & 0,007382 & 0,967252 & 0,002960 & 0,967524 & 0,003945 & 0,967488 & 0,004247 \\
\hline 54 & 0,000351 & 0,968033 & 0,002881 & 0,968177 & 0,003884 & 0,968105 & 0,004215 \\
\hline 55 & 0,009492 & 0,969346 & 0,002746 & 0,969182 & 0,003747 & 0,969202 & 0,004128 \\
\hline 56 & 0,005097 & 0,969506 & 0,002727 & 0,969271 & 0,003738 & 0,969243 & 0,004119 \\
\hline 57 & 0,010546 & 0,970281 & 0,002634 & 0,970034 & 0,003658 & 0,970115 & 0,001039 \\
\hline 58 & 0,000703 & 0,969676 & 0,002708 & 0,969200 & 0,003756 & 0,969266 & 0,004165 \\
\hline 59 & 0,034100 & 0,969676 & 0,002708 & 0,969200 & 0,003756 & 0,969266 & 0,004165 \\
\hline 60 & 0,007382 & 0,972263 & 0,002634 & 0,971678 & 0,003160 & 0,971587 & 0,003574 \\
\hline 61 & 0,041483 & 0,970770 & 0,003152 & 0,970131 & 0,003179 & 0,970085 & 0,003675 \\
\hline 62 & 0,004394 & 0,971725 & 0,003072 & 0,971486 & 0,003081 & 0,971275 & 0,003313 \\
\hline 63 & 0,025663 & 0,972224 & 0,003009 & 0,972104 & 0,003004 & 0,971926 & 0,003209 \\
\hline 64 & 0,007910 & 0,973132 & 0,003348 & 0,972976 & 0,002663 & 0,972734 & 0,002950 \\
\hline 65 & 0,000175 & 0,974164 & 0,003234 & 0,973720 & 0,002580 & 0,973446 & 0,002873 \\
\hline 66 & 0,007382 & 0,973737 & 0,003287 & 0,972738 & 0,002679 & 0,972449 & 0,002979 \\
\hline 67 & 0,000878 & 0,977027 & 0,002943 & 0,976431 & 0,002366 & 0,976164 & 0,002784 \\
\hline 68 & 0,004570 & 0,977027 & 0,002943 & 0,976431 & 0,002366 & 0,976164 & 0,002784 \\
\hline 69 & 0,009667 & 0,976585 & 0,002997 & 0,975813 & 0,002442 & 0,975551 & 0,002849 \\
\hline 70 & 0,019862 & 0,973581 & 0,003417 & 0,974021 & 0,002626 & 0,973702 & 0,002983 \\
\hline 71 & 0,027245 & 0,969196 & 0,003710 & 0,969632 & 0,003184 & 0,969337 & 0,003301 \\
\hline 72 & 0,024960 & 0,973883 & 0,003333 & 0,973796 & 0,002642 & 0,973644 & 0,002912 \\
\hline 73 & 0,002988 & 0,973795 & 0,003707 & 0,974399 & 0,002466 & 0,974165 & 0,002196 \\
\hline 74 & 0,009667 & 0,974812 & 0,003607 & 0,974366 & 0,002421 & 0,974190 & 0,002132 \\
\hline 75 & 0,025312 & 0,971096 & 0,004233 & 0,971855 & 0,002637 & 0,971735 & 0,002392 \\
\hline 76 & 0,004746 & 0,973411 & 0,003750 & 0,973842 & 0,002614 & 0,973862 & 0,002349 \\
\hline 77 & 0,011601 & 0,972781 & 0,003824 & 0,973310 & 0,002669 & 0,973294 & 0,002384 \\
\hline 78 & 0,000703 & 0,972781 & 0,003824 & 0,973310 & 0,002669 & 0,973294 & 0,002384 \\
\hline 79 & 0,000175 & 0,970067 & 0,004622 & 0,970929 & 0,002839 & 0,970841 & 0,002612 \\
\hline 80 & 0,019687 & 0,969470 & 0,004574 & 0,970929 & 0,002825 & 0,970989 & 0,002557 \\
\hline 81 & 0,001933 & 0,969470 & 0,004574 & 0,970929 & 0,002825 & 0,970989 & 0,002557 \\
\hline 82 & 0,023202 & 0,966884 & 0,001651 & 0,968377 & 0,002984 & 0,968375 & 0,002823 \\
\hline 83 & 0,019862 & 0,967490 & 0,004578 & 0,969211 & 0,002886 & 0,969224 & 0,002696 \\
\hline 84 & 0,004042 & 0,962433 & 0,005474 & 0,964988 & 0,003507 & 0,965015 & 0,003190 \\
\hline 85 & 0,016698 & 0,957672 & 0,006218 & 0,961100 & 0,003967 & 0,961100 & 0,003312 \\
\hline 86 & 0,009492 & 0,960470 & 0,005720 & 0,962532 & 0,003951 & 0,962566 & 0,003328 \\
\hline 87 & 0,034100 & 0,960470 & 0,005720 & 0,962532 & 0,003951 & 0,962566 & 0,003328 \\
\hline 88 & 0,011074 & 0,958635 & 0,005833 & 0,960633 & 0,004051 & 0,960589 & 0,003621 \\
\hline 89 & 0,000527 & 0,958635 & 0,005833 & 0,960633 & 0,004051 & 0,960589 & 0,003621 \\
\hline 90 & 0,000527 & 0,960331 & 0.005727 & 0,961699 & 0,003982 & 0,961506 & 0,003543 \\
\hline 91 & 0,009843 & 0,959682 & 0,005932 & 0,961813 & 0,004009 & 0,961707 & 0,003528 \\
\hline 92 & 0,015644 & 0,957005 & 0,006091 & 0,959721 & 0,004267 & 0,959610 & 0,003759 \\
\hline 93 & 0,001757 & 0,957431 & 0,006037 & 0,960702 & 0,004168 & 0,960607 & 0,003653 \\
\hline 94 & 0,009140 & 0,961669 & 0,005345 & 0,963839 & 0,003895 & 0,963818 & 0,003333 \\
\hline 95 & 0,006152 & 0,957666 & 0,005873 & 0,960666 & 0,004087 & 0,960764 & 0,003842 \\
\hline 96 & 0,010019 & 0,957043 & 0,005948 & 0,959739 & 0,004194 & 0,959862 & 0,003929 \\
\hline 97 & 0,002636 & 0,955996 & 0,006023 & 0,959280 & 0,004243 & 0,959538 & 0,003925 \\
\hline 98 & 0,009843 & 0,953419 & 0,006078 & 0,956616 & 0,004367 & 0,956957 & 0,003935 \\
\hline 99 & 0,005800 & 0,956044 & 0,005997 & 0,959261 & 0,004199 & 0,959775 & 0,003519 \\
\hline 100 & 0,005449 & 0,954806 & 0,006127 & 0,957982 & 0,004307 & 0,958532 & 0,003613 \\
\hline \multirow[t]{2}{*}{ POPOLACAO } & & $\operatorname{ESP}(r)$ & $\nabla 2 B(r)$ & $\operatorname{ESP}(r)$ & $\operatorname{VAR}(r)$ & $E S P(r)$ & $\operatorname{VAR}(r)$ \\
\hline & 1,000000 & 0,959792 & 0,004689 & 0,961088 & 0,004069 & 0,961218 & 0,003979 \\
\hline
\end{tabular}


QUADRO 5.5- PICIO DO ESTILADOR-RAZAO SEGUNDO O IETODO DE MNOSTRAGD PARA AS 4 POPULACOES

\begin{tabular}{llll} 
& & & \\
& PAI & $58 \mathrm{~S}$ & SILVA \\
\hline POPULACAO 1 & $-0,000979$ & 0,000010 & $-0,000113$ \\
POPULACAO 2 & $-0,013054$ & $-0,013485$ & $-0,013306$ \\
POPULACAO 3 & $-0,004613$ & $-0,002288$ & $-0,002402$ \\
POPULACAO 4 & 0,002424 & 0,003720 & 0,003850 \\
\hline
\end{tabular}

QUADBO 5.6- ERRO QUADRATICO IEDIO DO ESTIMADOR-RAZAO SEGUHDO 0 AETODO DE ALOSTRIGEI PARA AS \& POPULACOES

\begin{tabular}{llll} 
& & & \\
& PAI & $S \& \mathrm{~V}$ & SILDA \\
\hline POPULACAO 1 & 0,022135 & 0,021780 & 0,020960 \\
POPULACAO 2 & 0,017272 & 0,016404 & 0,016715 \\
POPULACAO 3 & 0,028663 & 0,025747 & 0,026521 \\
POPOLACAO 4 & 0,004695 & 0,004083 & 0,003994 \\
\hline
\end{tabular}


QUADRO 5.7-TESTE DE KRUSKAL-UALLIS

DIFEREACA/IETODO

\begin{tabular}{lrr}
\hline IETODO & \multicolumn{1}{l}{$\begin{array}{l}\text { RUIERO } \\
\text { DE CASOS }\end{array}$} & POSTO IEDIO \\
\hline PAI & 334 & 499,10 \\
$S \& D$ & 334 & 502,19 \\
SILVA & 334 & 503,21 \\
\hline Qui-quadrado & & 0,0365 \\
Iivel descritivo & 0,9819
\end{tabular}


QUADBO 5.8- TESTE DE XRUSKAL-DALLIS

DIFEREMCA/AETODO/TAXA DE RESPOSTA

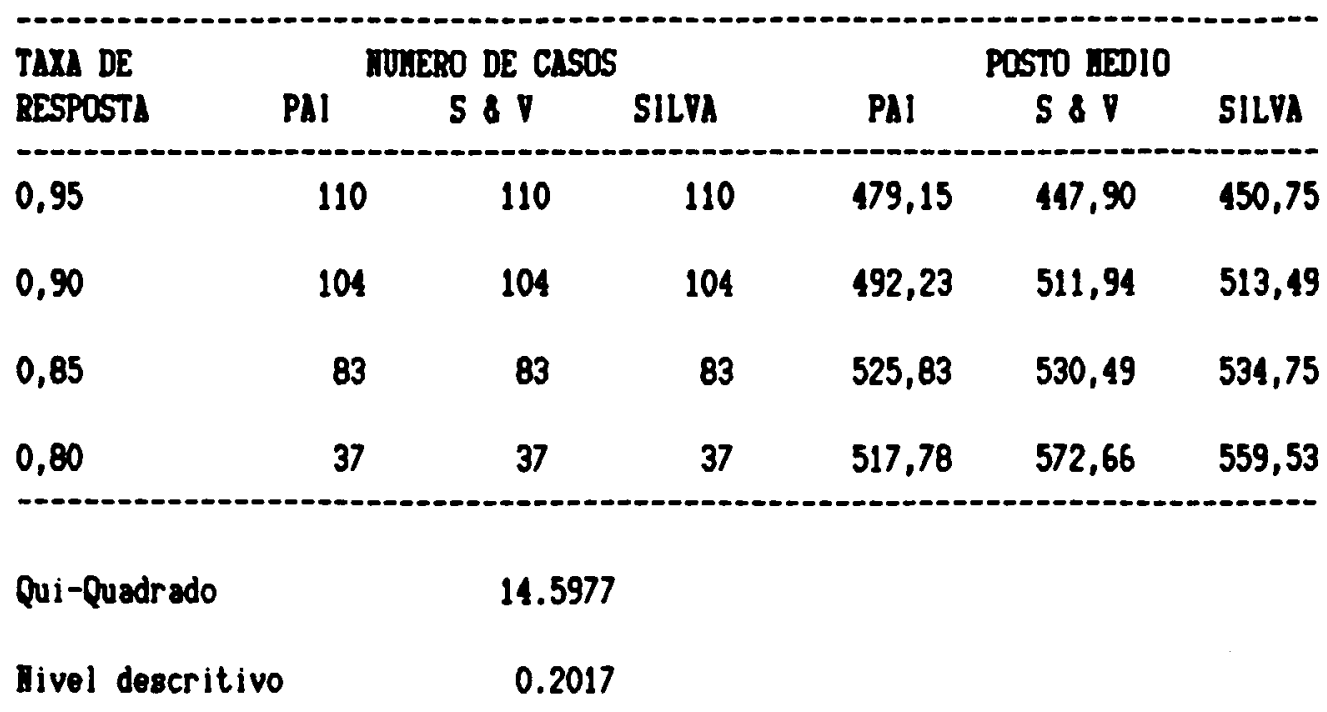

QULDBO 5.9 -TESTE DE KROSKAL-DALLIS

DIFEREMCA/AETODO/COBERTURA VACIRAL

\begin{tabular}{|c|c|c|c|c|c|c|}
\hline \multirow{2}{*}{$\begin{array}{l}\text { COBERTORA } \\
\text { VACIMAL }\end{array}$} & \multicolumn{3}{|c|}{ MULERO DE CASOS } & \multicolumn{3}{|c|}{ POSTO IEDIO } \\
\hline & PAI & $S \& V$ & SILVA & PAI & $S \& \nabla$ & SILVA \\
\hline $0,034483-0,227587$ & 29 & 29 & 29 & 568,14 & 573,71 & 591,76 \\
\hline $0,227588-0,420691$ & 27 & 27 & 27 & 409,06 & 534,26 & 525,43 \\
\hline $0,420692-0,613795$ & 64 & 64 & 64 & 461,81 & 514,31 & 502,85 \\
\hline $0,613796-0,806899$ & 88 & 88 & 88 & 526,66 & 460,90 & 468,06 \\
\hline $0,806900-1,000000$ & 126 & 126 & 126 & 502,20 & 501,53 & 502,80 \\
\hline Qui-Quadrado & $14 .{ }^{\circ}$ & & & & & \\
\hline Nivel deseritivo & & & & & & \\
\hline
\end{tabular}


QUADRO 5.10-TESTE DE RROSKAL-UALLIS

DIFEREMCA/AETODO/DERSIDADE IMTRADOHICILIAR DE CRIARCAS

\begin{tabular}{|c|c|c|c|c|c|c|}
\hline \multirow{2}{*}{$\begin{array}{l}\text { DEMSIDLDE IRTRADOMI- } \\
\text { CILLIAR DE CRIANCAS }\end{array}$} & \multicolumn{3}{|c|}{ MULERO DE CASOS } & \multicolumn{3}{|c|}{ POSTO IEDIO } \\
\hline & PAI & $S \& V$ & SILVA & PAI & $5 \& V$ & SILVA \\
\hline $0,0716670-0,1060002$ & 45 & 45 & 45 & 504,49 & 465,59 & 472,69 \\
\hline $0,1060003-0,1403334$ & 163 & 163 & 163 & 489,08 & 494,48 & 497,35 \\
\hline $0,1403335-0,1746666$ & 101 & 101 & 101 & 492,91 & 499,00 & 502,60 \\
\hline $0,1746667-0,2089998$ & 24 & 24 & 24 & 570,46 & 624,33 & 594,33 \\
\hline $0,2089999-0,243333$ & 1 & 1 & 1 & 803,00 & 797,00 & 706,00 \\
\hline
\end{tabular}

Qui-Quadrado

12.4619

Rivel descritivo

0.5693

QOADRO 5.11-TESTE DE BRUSKAL-DALLIS

DIFEREACA/AETODO/TAMARHO DO COMGLOHERADO

\begin{tabular}{|c|c|c|c|c|c|c|}
\hline \multirow{2}{*}{$\begin{array}{l}\text { TAHAMHO DO } \\
\text { COHGLOHERADO }\end{array}$} & \multicolumn{3}{|c|}{ MULERO DE CLSOS } & \multicolumn{3}{|c|}{ POSTO IEDIO } \\
\hline & PAI & $5 \& 7$ & SILVA & PAI & $S \& V$ & SILVA \\
\hline 200 & 75 & 75 & 75 & 505,71 & 534,44 & 528,59 \\
\hline 300 & 107 & 107 & 107 & 501,86 & 456,36 & 457,85 \\
\hline 450 & 88 & 88 & 88 & 505,41 & 491,14 & 495,47 \\
\hline 600 & 64 & 64 & 64 & 478,07 & 556,21 & 559,95 \\
\hline
\end{tabular}

$\begin{array}{lr}\text { Qui-Quadrado } & 12.1751 \\ \text { Iivel deacritivo } & 0.3506\end{array}$




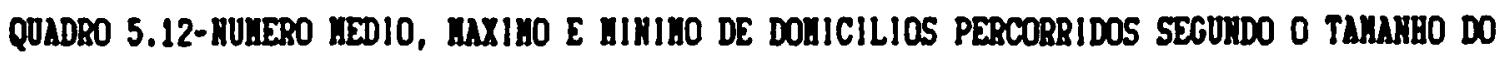
COMGLOAERADO PARA OBTER OAA SUBAHOSTRA DE SETE CRILICAS PELO IETODO PAI PARA CLDA POPULACAO

POPULACAO 1 POPULACAO 2 POPULACAO 3 POPULACAO 4 CORGLOAERADO

\begin{tabular}{|c|c|c|c|c|c|}
\hline 200 & $\begin{array}{l}\text { IEDIA } \\
\text { Maxiluo } \\
\text { MInISO }\end{array}$ & $\begin{array}{r}58,9365 \\
133 \\
9\end{array}$ & $\begin{array}{r}56,3675 \\
133 \\
9\end{array}$ & $\begin{array}{r}58,4647 \\
119 \\
13\end{array}$ & $\begin{array}{r}59,1534 \\
146 \\
11\end{array}$ \\
\hline 300 & $\begin{array}{l}\text { MEDI } \\
\text { WAXILO } \\
\text { MIMIHO }\end{array}$ & $\begin{array}{r}57,5287 \\
142 \\
7\end{array}$ & $\begin{array}{r}56,9635 \\
112 \\
9\end{array}$ & $\begin{array}{r}58,2620 \\
173 \\
10\end{array}$ & $\begin{array}{r}58,7009 \\
165 \\
9\end{array}$ \\
\hline 450 & $\begin{array}{l}\text { IEDIA } \\
\text { HAXIMO } \\
\text { MINILO }\end{array}$ & $\begin{array}{r}62,2924 \\
197 \\
9\end{array}$ & $\begin{array}{r}62,9112 \\
197 \\
8\end{array}$ & $\begin{array}{r}61,2144 \\
179 \\
6\end{array}$ & $\begin{array}{r}62,0672 \\
164 \\
10\end{array}$ \\
\hline 600 & $\begin{array}{l}\text { IIEDIA } \\
\text { MaxIno } \\
\text { MINIHO }\end{array}$ & $\begin{array}{r}61,2237 \\
164 \\
10\end{array}$ & $\begin{array}{r}59,7042 \\
164 \\
10\end{array}$ & $\begin{array}{r}60,2172 \\
196 \\
10\end{array}$ & $\begin{array}{r}61,7665 \\
158 \\
13\end{array}$ \\
\hline
\end{tabular}




\section{CONCLUSÕES}

1) Os métodos de Henderson e Sundaresan e de Szwarcwald e Valente, embora não sejam equiprobabilísticos em condições normais, produzem resultados que não se distanciam significativamente do método de Silva para as diversas situações simuladas.

2) 0 método de Henderson e Sundaresan, que é reconhecidamente de simples aplicação e custo reduzido, é uma alternativa recomendada para levantamentos de cobertura vacinal pois se adequa perfeitamente às necessidades dos serviços de saúde dos países em desenvolvimento, que precisam de informações confiáveis, produzidas com baixo custo. 


\section{Referências Bibliográficas}

1 COCHRAN, W.C. Sampling Techniques. 3rd. ed. New York, John Wiley E Sons, $197 \%$.

2 FUNDAÇÃO IBGE. Tabulações do censo demográfico. Rio de Janeiro, 1981. (9. Recenseamento Geral do Brasil, 1980).

3 HASTINGS, N.A.J. \& PEACOCK, J.B..Statistical Distributions, London, Butterworth \& Co (Publishers) Ltda., 1975.

4 HENDERSON, R.H. et al.Assessment of vaccination coverage, vaccination scar rates, and smallpox scarring in five areas of West Africa. Bull. Wld Hlth Org., 48: 183-194, 1973.

5 HENDERSON, R.H. \& SUNDARESAN, T. Cluster sampling to assess immunization coverage : a review of experience with a simplified sampling method. Bull. Wld Hlth Org., 60(2) : 253-260, 1982.

6 KALTON, G. Introduction to survey sampling. Beverly Hills, Sage University, 1983. (Paper Series on Quantitative Application in the Social Sciences, n. 07-035).

7 KISH, L. Survey Sampling. New York, John Wiley \& Sons, 1965.

8 LAURENTI; R. et al. Estatísticas de saúde. $2^{a}$ ed. São Paulo, Editora Pedagógica e Universitária Ltda., 1980.

9 LEMESHOW, S. et al.A computer simulation of the EPI survey strategy. Int. J. Epidem., 14: 473-481, 1985.

10 LEMESHOW, S. \& ROBINSON, D. Surveys to measure programme coverage and impact: a review of the methodology used by the expanded programme on immunization. Wld Hlth Statist. Quart., 38.: 65-75, 1985.

11 LIGUE FRANÇAISE POUR LA PREVENTION DES MALADES INFECTIEUSES. Réunion sur la couverture vaccinale. 5 fev. 1983. [Rapport]. Paris, 1983 [mimeografado] 
12 LWANGA, S.K. \& ABIPROJO; N.Immunization coverage surveys: methodological studies in Indonesia. Bull. Wld H]th Org., 65: 847-853, 1987.

13 NAKAO, N.Avaliação da cobertura vacinal. São Paulo, 1987. Dissertação de Mestrado - Faculdade de Saúde Pública

14 NAYLOR, T.H. et al Técnicas de simulação em computadores. São Paulo, Vozes/Editora da USP, 1971

15 ORGANIZAÇÃO PANAMERICANA DE SAÚDE.Encuestas sobre cobertura de inmunización. Bol. inform. PAI, 5(2): 1-2, 1983.

16 ROTHENBERG, R.B. et al.Observations on the application of EPI cluster survey methods for estimating disease incidence. Bull. Wld Hlth Org., 63(1): 93-99, 1985.

17 SALMASO, S. et al.Immunization coverage in Italy. Bull. Wld Hlth Org., 65: 841-846, 1987.

18 SERFLING, R. \& SHERMAN, L.L. Apud HENDERSON, R.H. et al. Assesment of vacination coverage, vaccination scar rates, and smallpox scarring in five areas of West Africa. Bull. Wld Hlth Org., 48: 183194, 1973.

19 SIEGEL, S. Estatística não-paramétrica. São Paulo, McGraw-Hill, 1975.

20 SILVA, E.P. de C.Métodos de amostragem para estimação da cobertura vacinal. Rev. Saúde públ., São Paulo, 20: 377-84, 1986.

21 SZWARCWALD, C.L. \& VALENTE, J.G.Avaliação da cobertura de vacinação em Teresina Piauí (Brasil - 1983). Cad. Saúde públ., Rio de Janeiro, 1: 41-9, 1985.

22 WORLD HEALTH ORGANIZATION.Expanded Programme on Immunization. Training for mid-level managers: evaluate vaccination coverage. Geneva, s.d. 


\section{ANEXOS}




\section{ANEXO 1 \\ Programas para a simulação dos conglomerados e das sub-amostras}




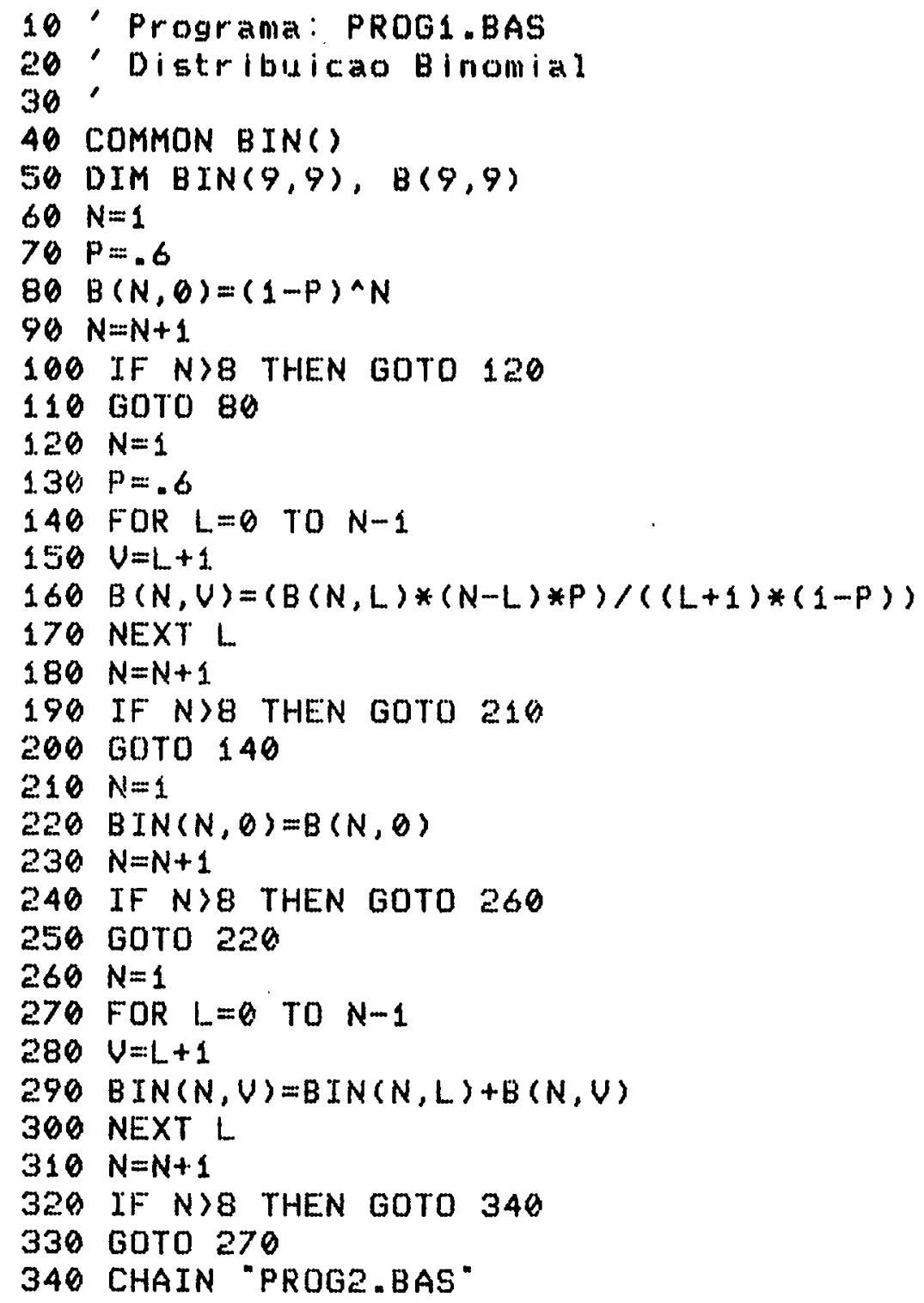




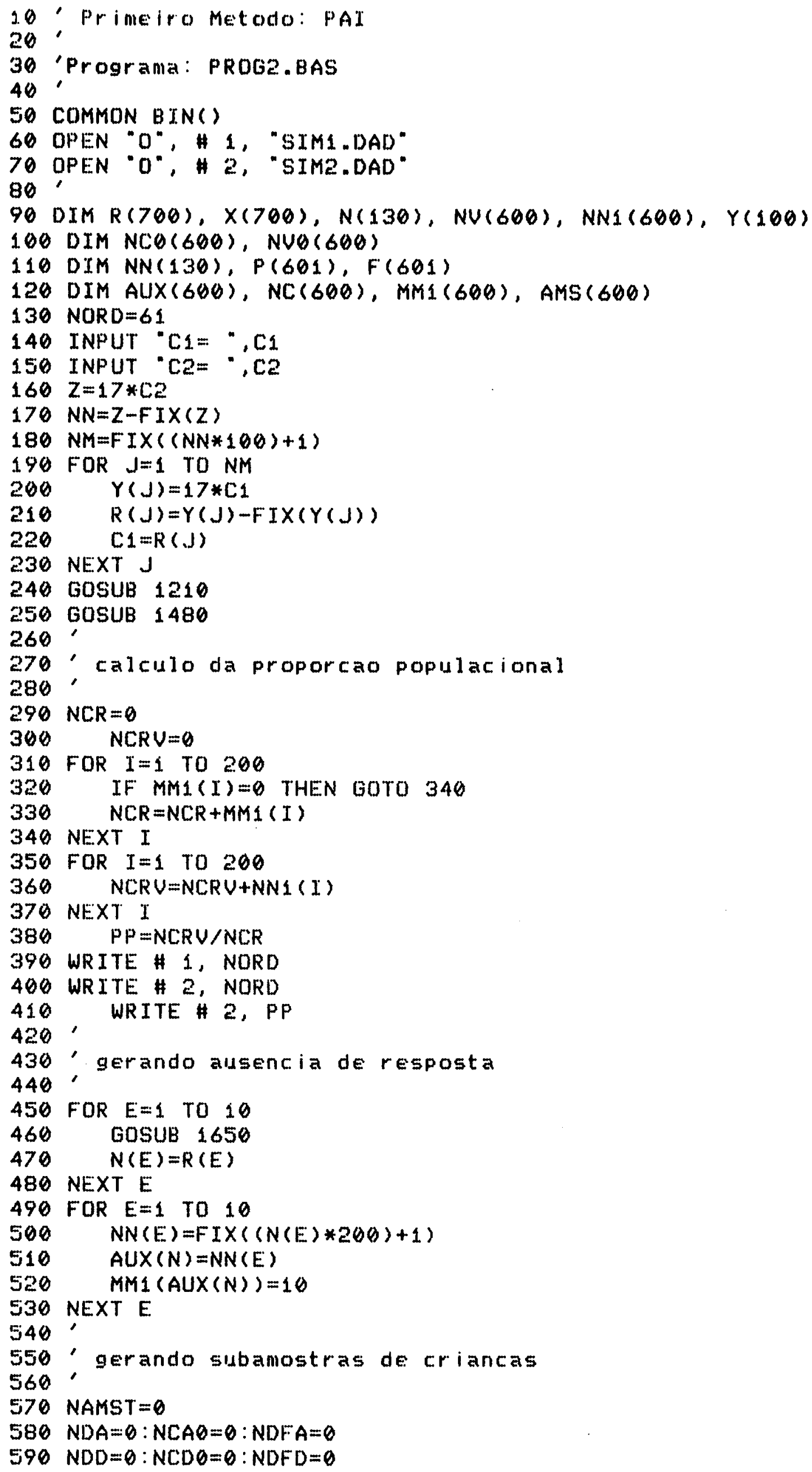




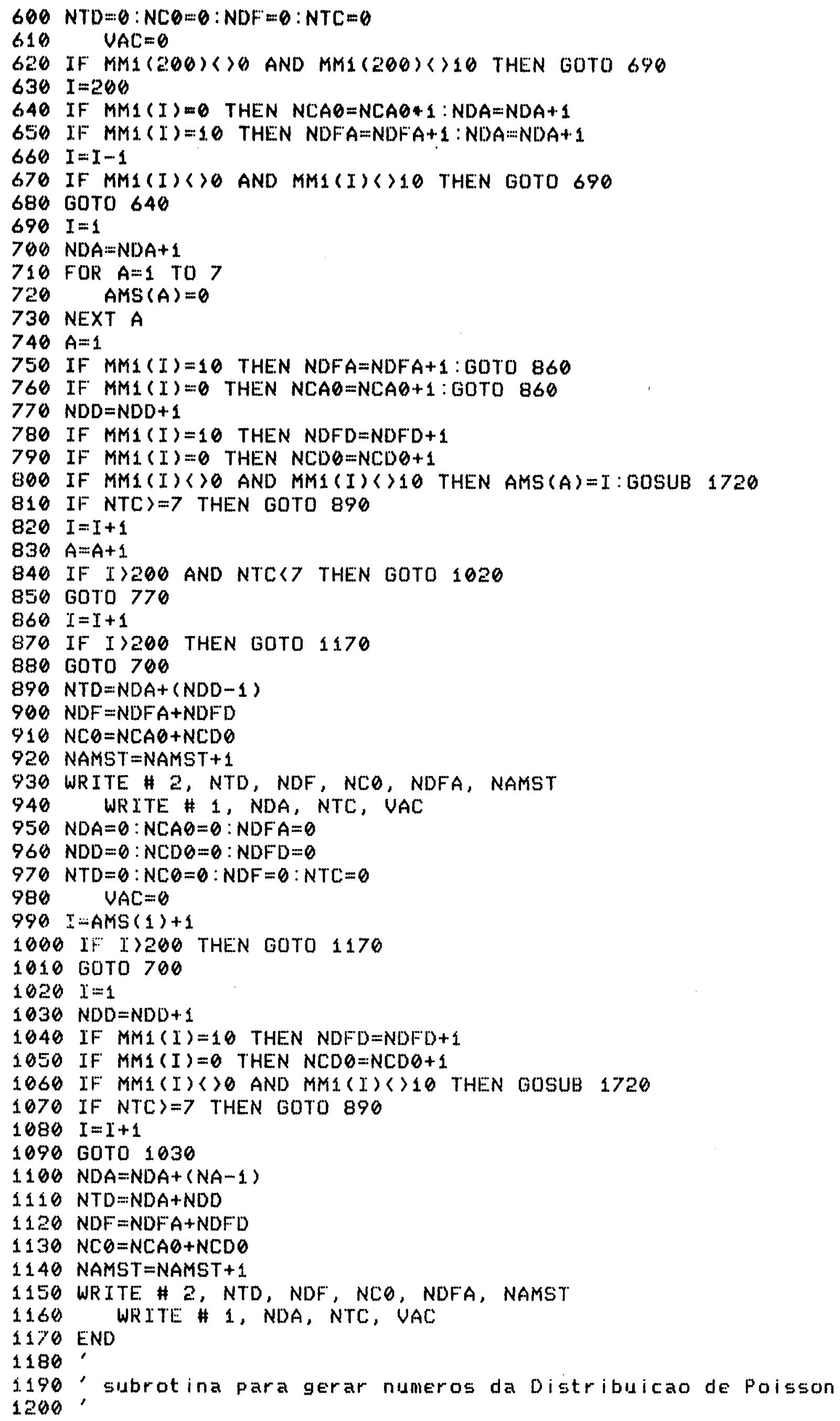




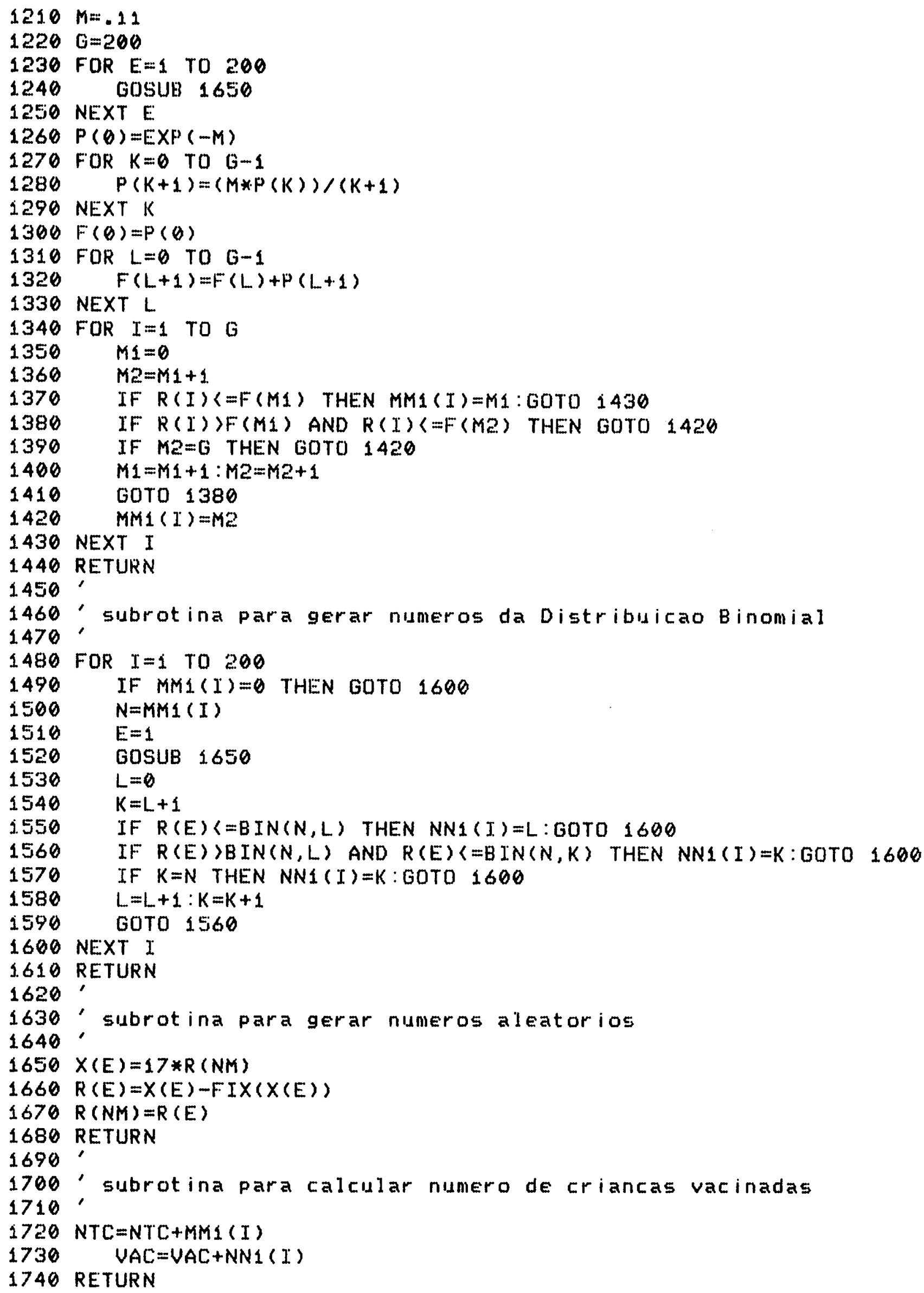




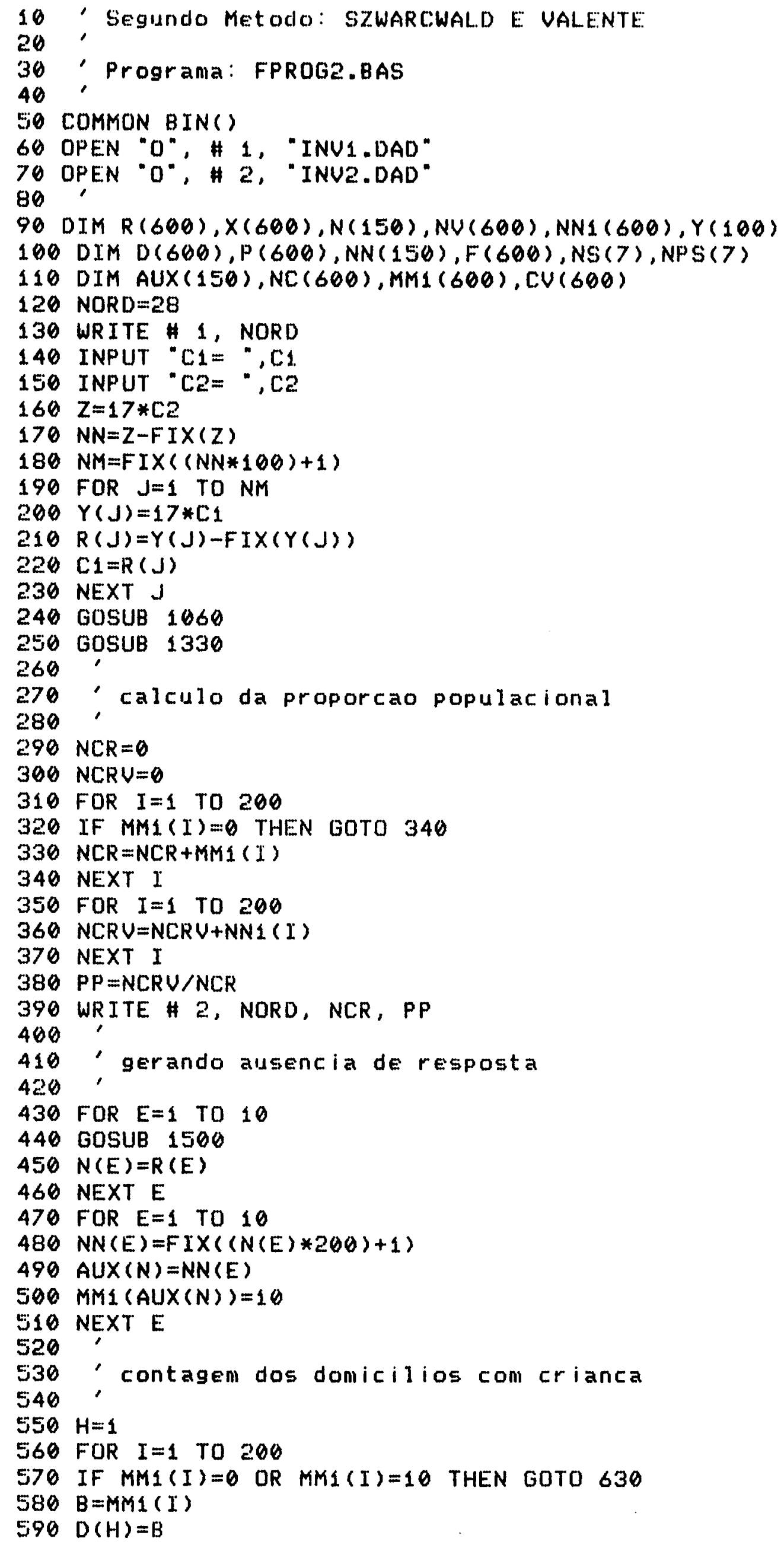




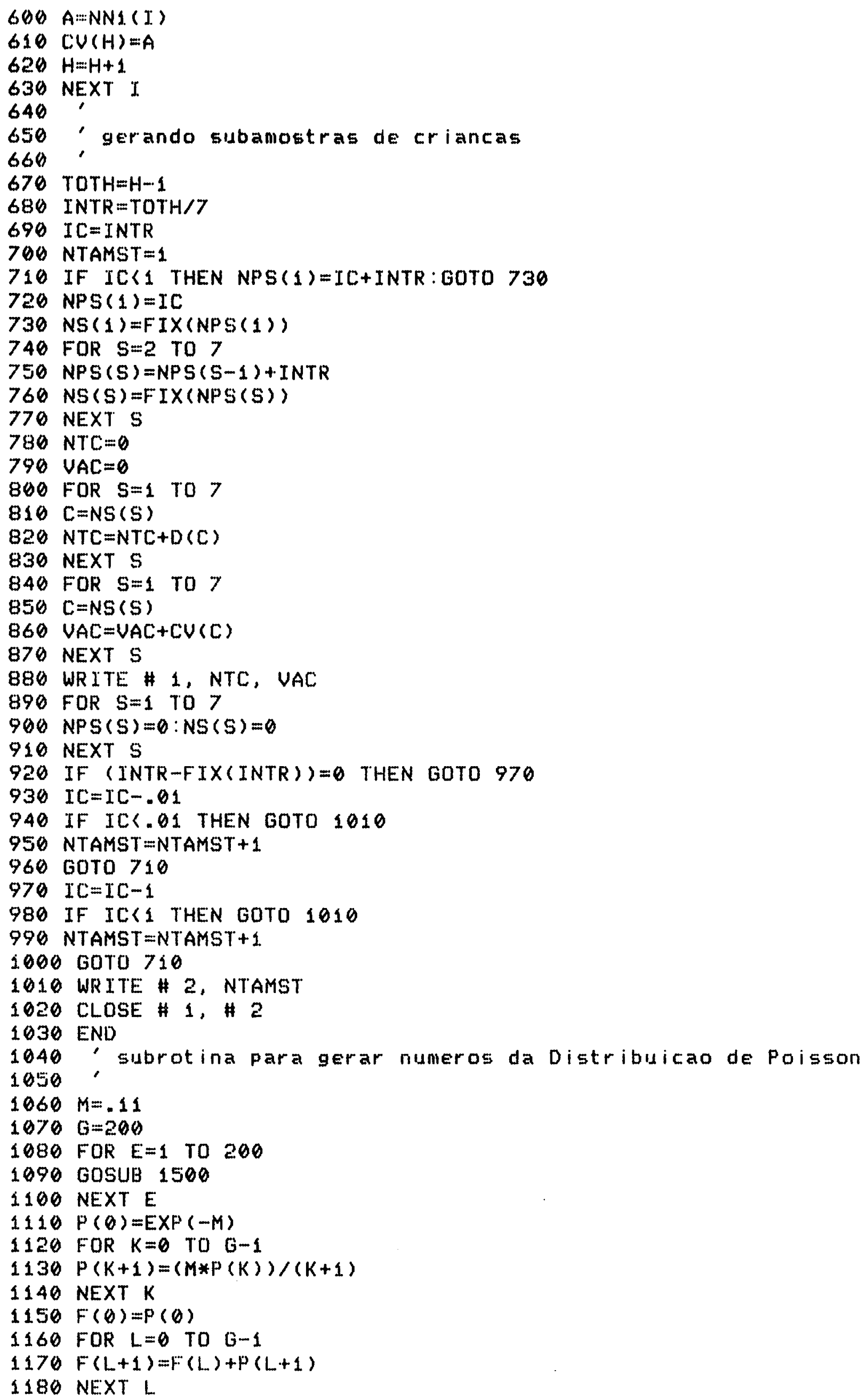




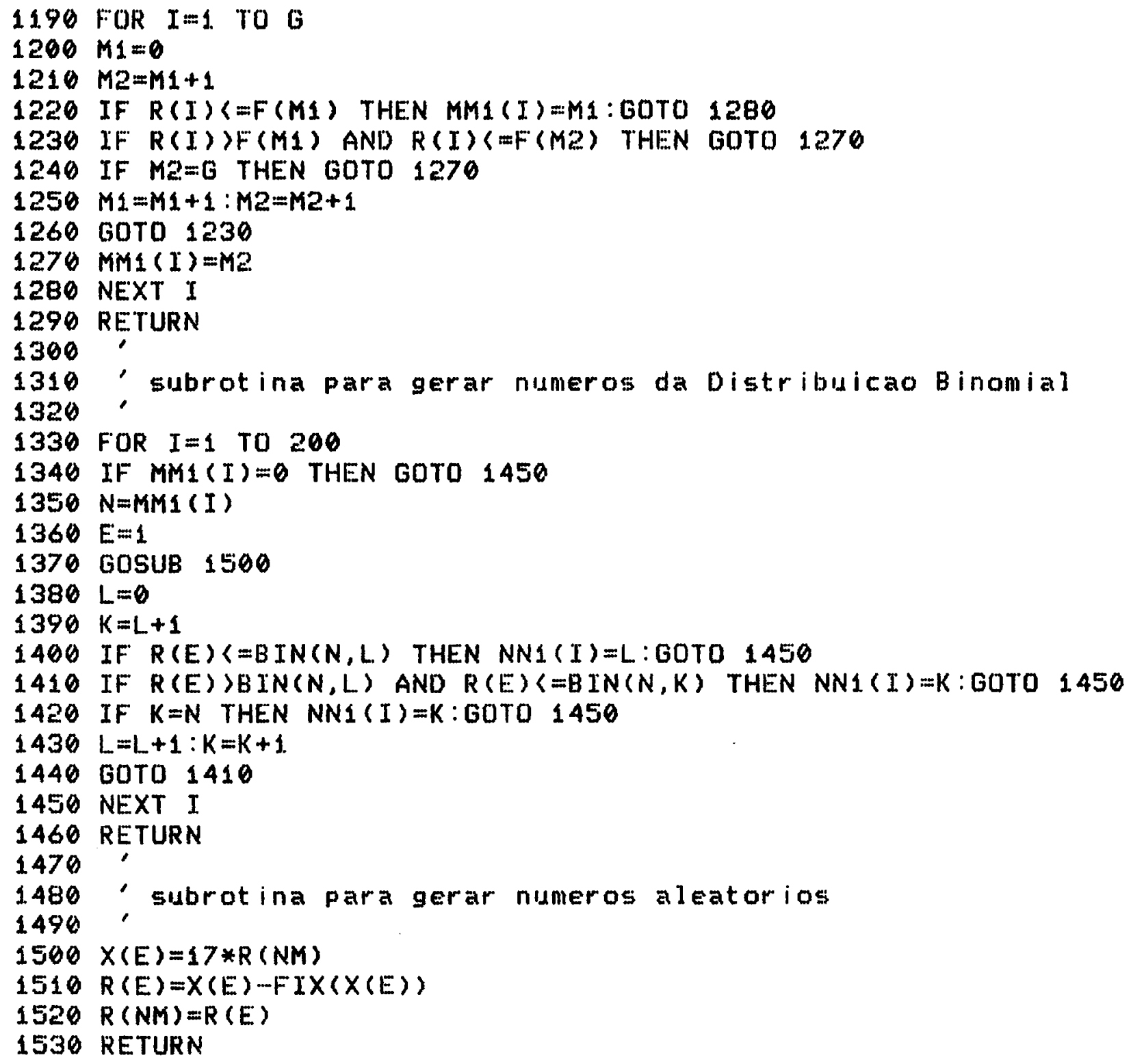




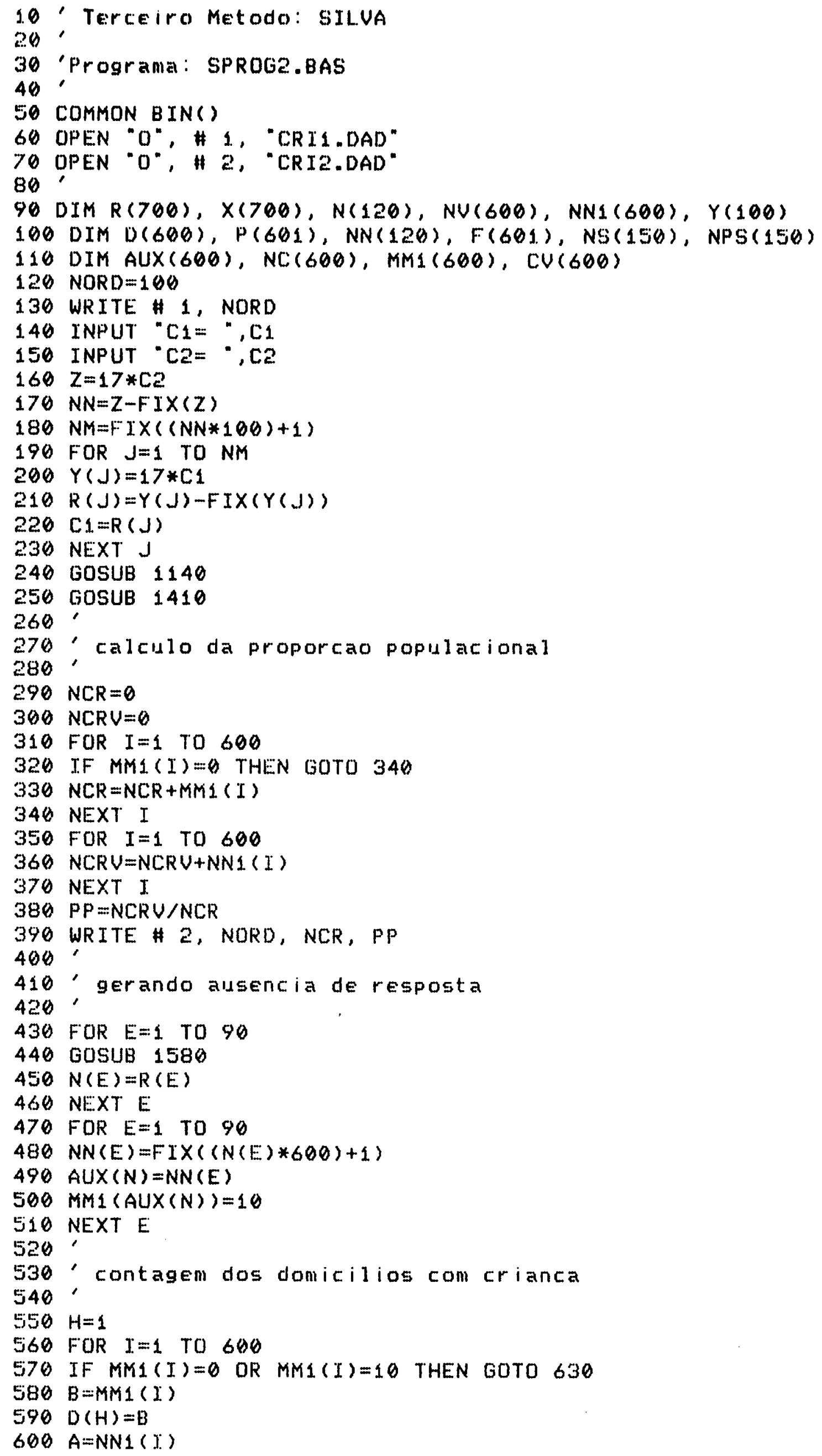




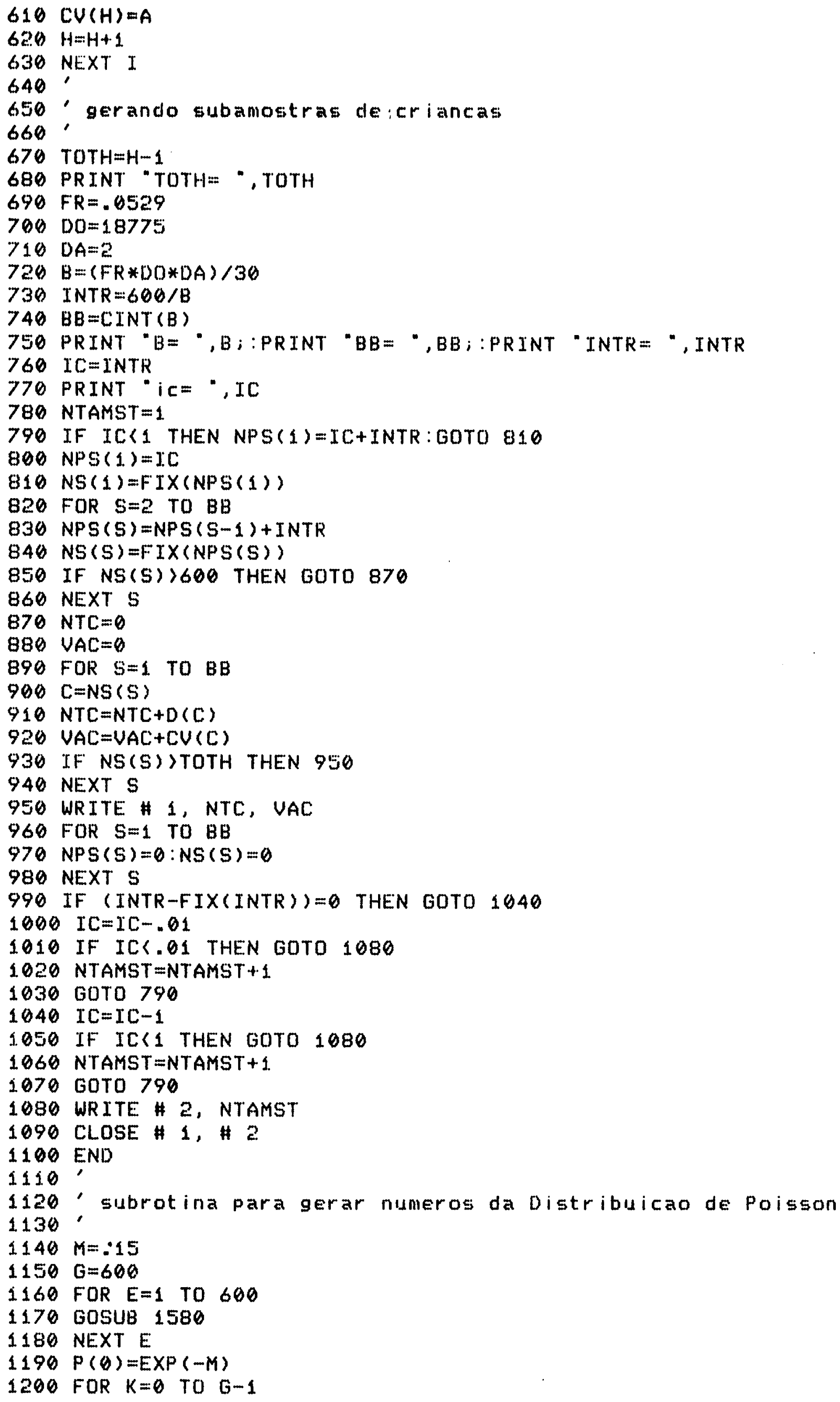




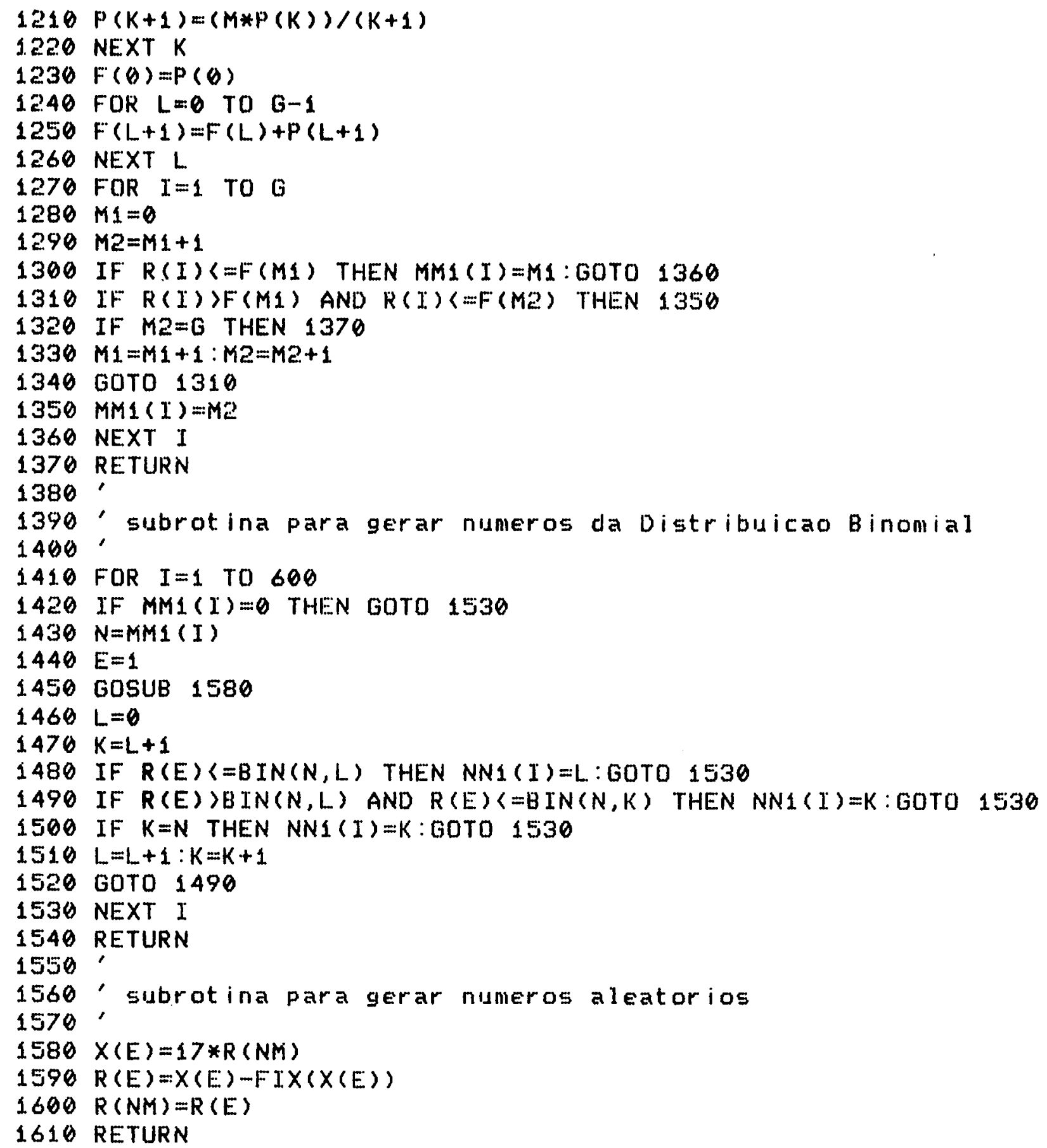


Program Sorteioi

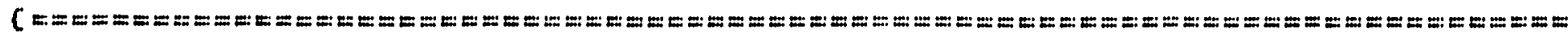

C Programa para sorteio de amostras

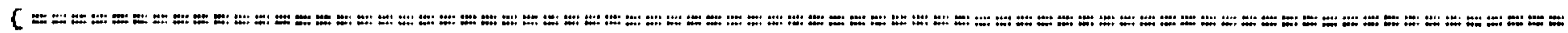

Const Ncaso $=30$;

$\operatorname{Max}=300 ;$

Type VetorR = arrayl.. 1. . Max] of real;

VetorI = array[. S... Maxd of integer;

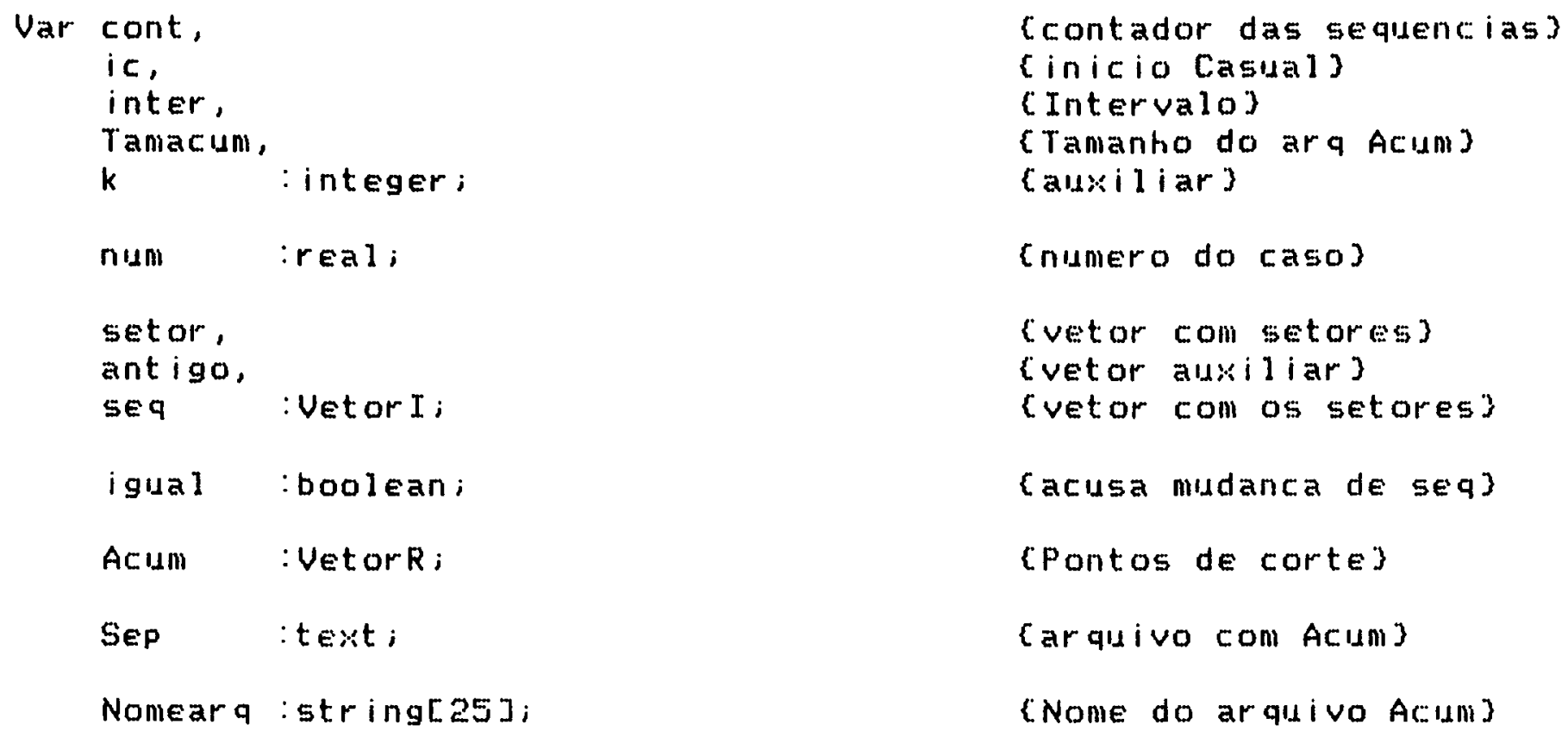

Function Orden (Num:Real;Acun: VetorR; Setor:VetorI; Tamacum:integer): integer;

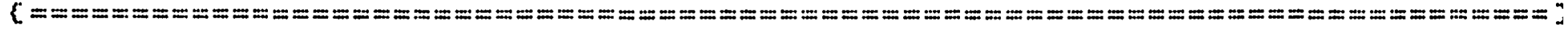
c Calcula o setor em que o Nun perttence

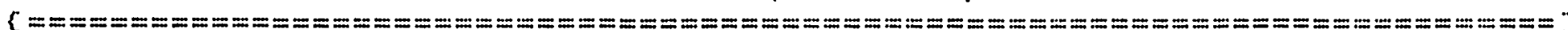

Var Apont I : integer;

\{Apontador para percorrer Acum\} [ACusa que encontrou o setior\}

Begin

Apont I : :=0;

Achoul := False;

While ((ApontI (Tamacum) and (not Achou)) do

beg in apont $I:=$ apont $I+1 ;$

if ((Acum[ApontI] (Num) and (Num (= Acun[ApontI + 1])) then Achou: = true end;

Orden: := setor[ApontI];

end;

Begin (Programa Principal)

Clrscr;

Got $0 \times y(20,12)$;

write ('Qual o arquivo com os valores Acunulados?');

readln (Nomear q);

Assign (Sep, Nonear $q$ );

reset ( $S \in P$ );

readln (Sep, Tanacun);

For $k:=1$ to Tamacum do 


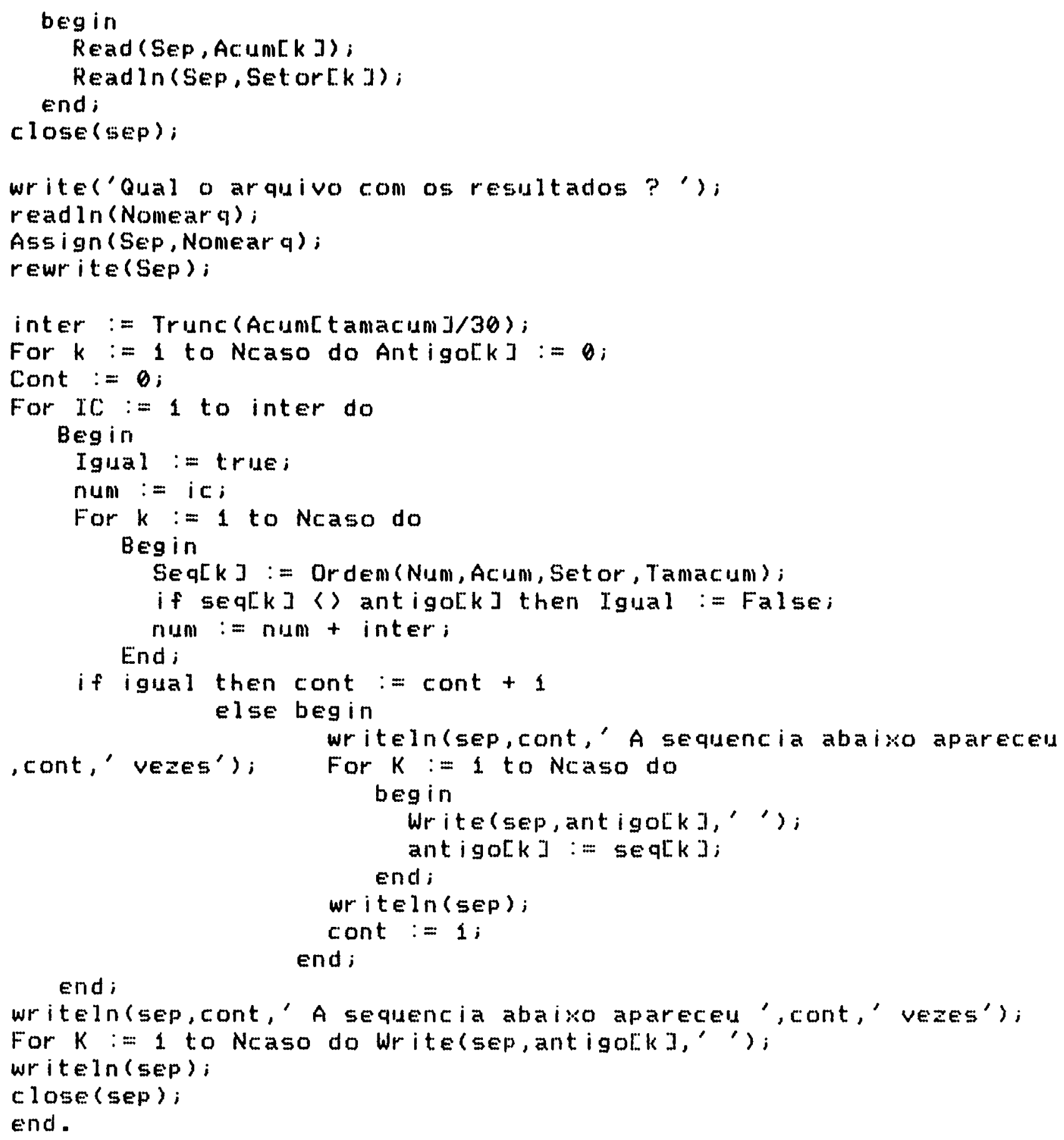




\section{ANEXO 2 \\ Programas para análise dos dados}




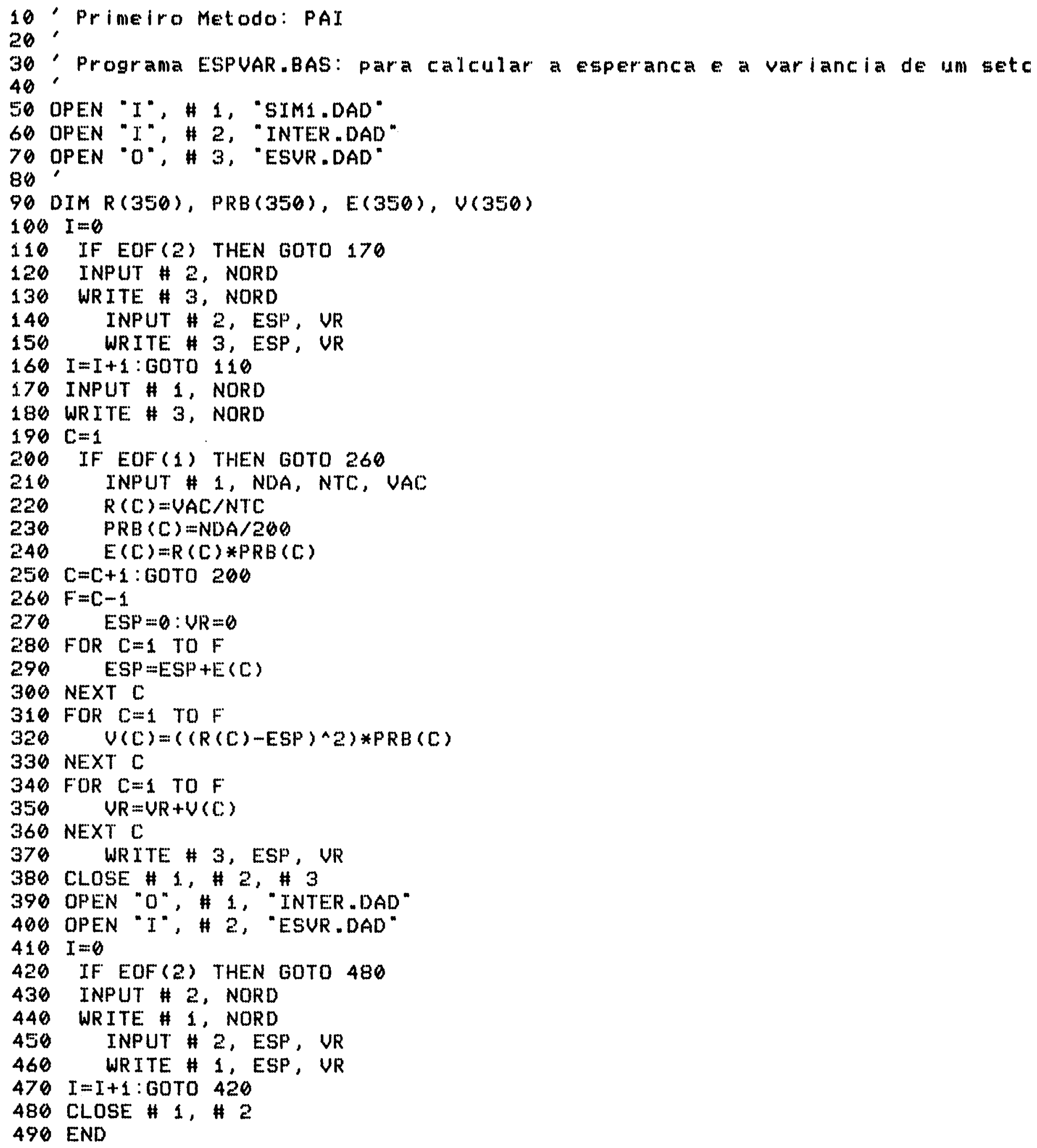


10 Segundo Metodo: SZWARCWALD E VALENTE

30 'Programa fESPUAR.BAS: para calcular a esperanca a variancia de un setol 40

50 OPEN "I", \#1, "INU1.DAD"

60 OPEN "I", \#2, "INTER.DAD"

70 OPEN " $0 "$ " \#3, "FESUR.DAD"

80

90 DIM R(300), E(300), U(300)

$100 I=0$

110 IF EOF (2) THEN GOTO 170

120 INPUT \#2, NORD

130 WRITE. \# 3 , NORD

140 INPUT \# 2, ESP, UR

150 WRITE \# 3 , ESP, UR

$160 I=I+1:$ GOTO 110

170 INPUT \#1, NORD

180 WRITE \#3, NORD

$190 \mathrm{C}=1$

200 IF EOF(1) THEN GOTO 240

210 INPUT \# 1, NTC, VAC

$220 R(C)=V A C / N T C$

$230 \mathrm{C}=\mathrm{C}+1:$ GOTO 200

$240 \mathrm{~F}=\mathrm{C}-1$

250 NTAMST $=F$

260 FOR $C=1$ TO $F$

$270 E(C)=R(C) *(1 / N$ TAMST $)$

280 NEXT C

$290 E S P=0: U R=0$

300 FOR $C=1$ TO $F$

$310 E S P=E S P+E(C)$

320 NEXT C

330 FOR $\mathrm{C}=1$ TO $\mathrm{F}$

$340 U(C)=((R(C)-E S P) \wedge 2) *(1 / N T A M S T)$

350 NEXT $C$

360 FOR $C=1$ TO $F$

370 UR $=U R+U(C)$

380 NEXT C.

390 WRITE \# 3 , ESP, UR

400 CLOSE \#1, \#2, \#3

410 OPEN "O", \#1., "INTER . DAD"

420 OPEN "I", \#2, "FESUR.DAD"

$430 I=0$

440 IF EOF (2) THEN GOTO 500

450 INPUT \#2, NORD

460 WRITE \#1, NORD

470 INPUT \# 2, ESP, UR

480 WRITE \#1, ESP，UR

$490 I=I+1:$ GOTO 440

500 CLOSE \# 1 , \# 2

510 END 
10 Terceiro Metodo: SILva

20

30' Programa SESPUAR.BAS: para calcular a esperanca a variancia de um getor

50 OPEN "I", \#1, "CRI1.DAD"

60 OPEN "I", \#2, "INTER.DAB"

70 OPEN "O", \#3, "SESUR.DAD"

80

90 DIM R $(2500), E(2500), U(2500)$

$100 I=0$

110 IF EOF (2) THEN GOTO 170

120 INPUT \#2, NORD

130 WRITE \# 3 , NORD

140 INPUT \# 2, ESP, UR

150 WRITE \# 3 , ESP, UR

$160 \quad I=I+1:$ GOTO 110

170 INPUT \#1, NORD

180 WRITE \#3, NORD

$190 \mathrm{C}=1$

200 IF EOF (1) THEN GOTO 240

210 INPUT \#1, NTC, VAC

$220 R(C)=V A C / N T C$

$230 \mathrm{C}=\mathrm{C}+1: \mathrm{GOTO} 200$

$240 \quad F=C-1$

250 NTAMST $=F$

260 FOR $C=1$ TO $F$

$270 E(C)=R(C) *(1 /$ NTAMST $)$

280 NEXT $C$

$290 \mathrm{ESP}=0: \mathrm{UR}=0$

300 FOR $C=1$ TO $F$

$310 E S P=E S P+E(C)$

320 NEXT C

330 FOR $C=1$ TO $F$

$340 U(C)=((R(C)-E S P) \wedge 2) *(1 / N T A M S T)$

350 NEXT $C$

360 FOR $\mathrm{C}=1$ TO $\mathrm{F}$

370 UR $=U R+U(C)$

380 NEXT C

390 WRITE \# 3, ESP, UR

400 CLOSE \#1, \#2, \#3

410 OPEN "0", \#1, "INTER.DAD"

420 OPEN "I", \#2, "SESUR.DAD"

$430 \mathrm{~J}=0$

440 IF EOF(2) THEN GOTO 500

450 INPUT \# 2, NORD

460 WRITE \#1, NORD

470 INPUT \#2, ESP, UR

480 WRITE \#1, ESP, UR

$490 I=I+1:$ GOTO 440

500 CLOSE \# 1 , \#2

510 END 


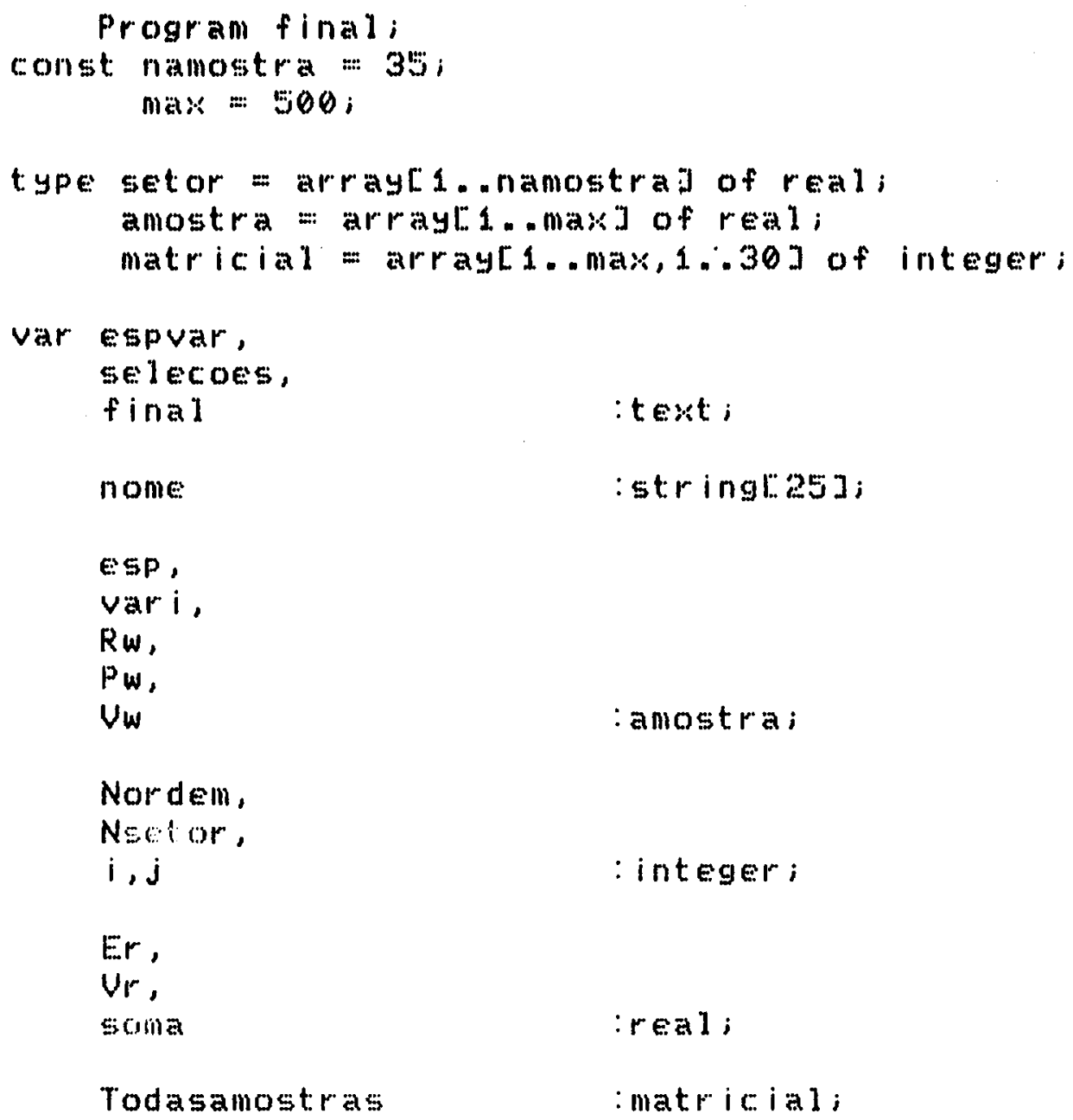




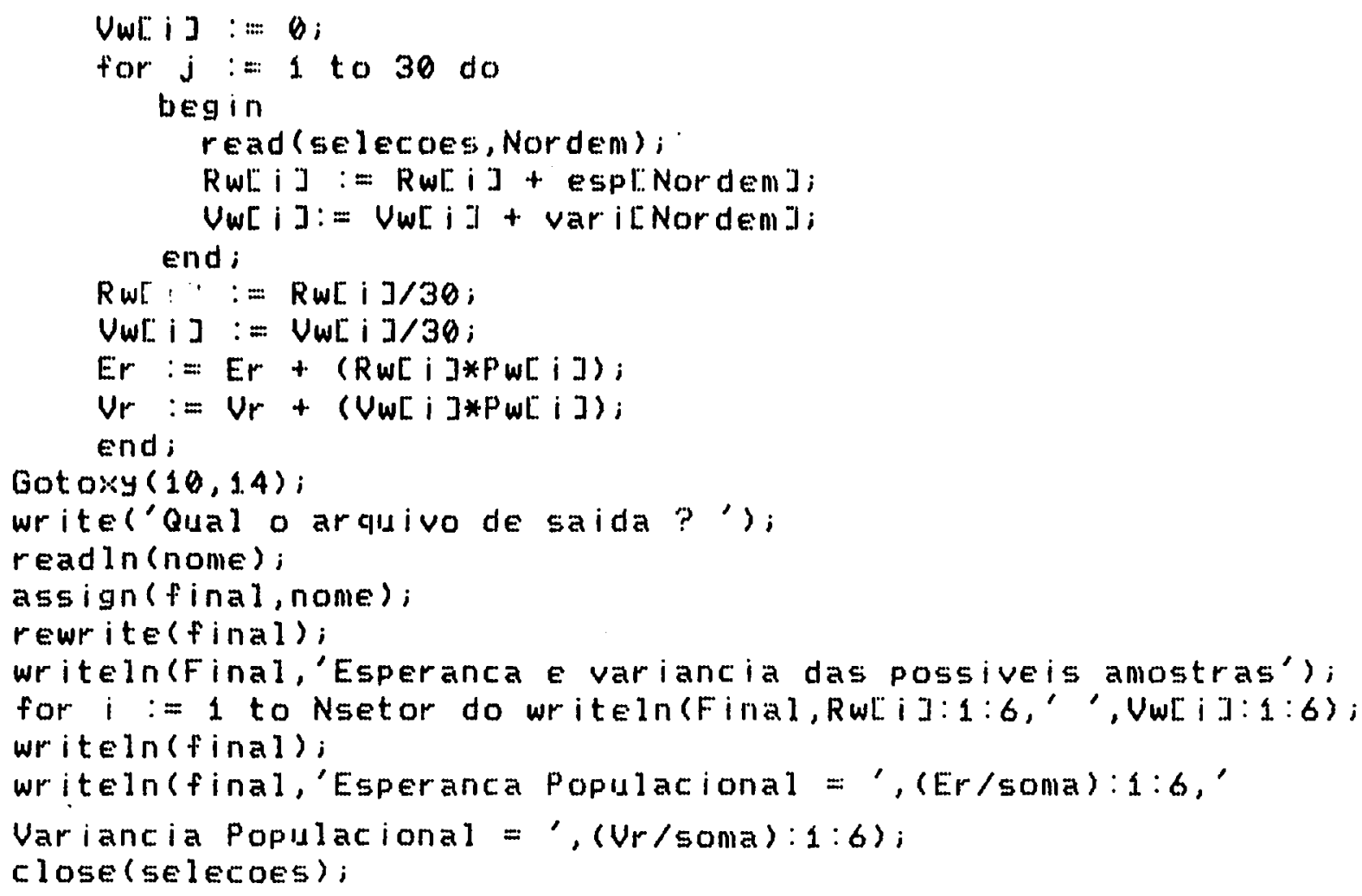

